Pacific

Journal of

Mathematics

\title{
A DENSITY THEOREM \\ IN PARAMETRIZED DIFFERENTIAL GALOIS THEORY
}

THOMAS DREYFUS

Volume $271 \quad$ No. 1

September 2014 


\title{
A DENSITY THEOREM IN PARAMETRIZED DIFFERENTIAL GALOIS THEORY
}

\author{
THOMAS DREYFUS
}

We study parametrized linear differential equations with coefficients depending meromorphically upon the parameters. As a main result, analogously to the unparametrized density theorem of Ramis, we show that the parametrized monodromy, the parametrized exponential torus and the parametrized Stokes operators are topological generators in the Kolchin topology for the parametrized differential Galois group introduced by Cassidy and Singer. We prove an analogous result for the global parametrized differential Galois group, which generalizes a result by Mitschi and Singer. These authors give also a necessary condition on a group for being a global parametrized differential Galois group; as a corollary of the density theorem, we prove that their condition is also sufficient. As an application, we give a characterization of completely integrable equations, and we give a partial answer to a question of Sibuya about the transcendence properties of a given Stokes matrix. Moreover, using a parametrized HukuharaTurrittin theorem, we show that the Galois group descends to a smaller field, whose field of constants is not differentially closed.

1. Local analytic linear differential systems depending upon parameters 92

1A. Definition of the fields 93

1B. The Hukuhara-Turrittin theorem in the parametrized case 95

1C. Review of the Stokes phenomenon in the unparametrized case 98

1D. Stokes phenomenon in the parametrized case 102

2. Parametrized differential Galois theory 105

2A. Basic facts 105

2B. Parametrized differential Galois theory for nonclosed fields 109

2C. Descent for the local analytic parametrized differential Galois group 113

2D. An analogue of the density theorem in the parametrized case 115

2E. Density theorem for the global parametrized differential Galois group 119

2F. Examples

Work partially supported by ANR, contract ANR-06-JCJC-0028.

MSC2010: 12H20, 34M15, 34M03.

Keywords: parametrized differential Galois theory, Stokes phenomenon. 
3. Applications

3A. Completely integrable equations

3B. On the hypertranscendence of a Stokes matrix

3C. Which linear differential algebraic groups are parametrized differential Galois groups?

Appendix

Acknowledgements

\section{Introduction}

Let us consider a linear differential system of the form

$$
\partial_{z} Y(z)=A(z) Y(z),
$$

where $\partial_{z}=d / d z$, and $A(z)$ is an $m \times m$ matrix whose entries are germs of meromorphic functions in a neighborhood of a point, say 0 to fix ideas. The differential Galois group, which measures the algebraic dependencies among the solutions, can be viewed as an algebraic subgroup of $\mathrm{GL}_{m}(\mathbb{C})$ via the injective group morphism

$$
\begin{aligned}
\rho_{U}: \mathrm{Gal} & \rightarrow \mathrm{GL}_{m}(\mathbb{C}), \\
\sigma & \mapsto U(z)^{-1} \sigma(U(z)),
\end{aligned}
$$

where $U(z)$ is some arbitrary fundamental solution, i.e., an invertible solution matrix.

Let $U(z)$ be a fundamental solution contained in a Picard-Vessiot extension of the equation $\partial_{z} Y(z)=A(z) Y(z)$. The linear differential equation is said to be regular singular at 0 if there exists an invertible matrix $P(z)$ whose entries are germs of meromorphic functions such that $W(z)=P(z) U(z)$ satisfies

$$
\partial_{z} W(z)=\frac{A_{0}}{z} W(z),
$$

where $A_{0}$ is a matrix with constant complex entries. In this case, $W(z)$ usually involves multivalued functions. Analytic continuation of $W(z)$ along any simple loop $\gamma$ around 0 yields another fundamental solution $W(z) M_{\gamma}$. The matrix $M_{\gamma}$, which is a monodromy matrix, has complex entries and depends only on the homotopy class of $\gamma$. The Schlesinger theorem says that the Zariski closure of the group generated by the monodromy matrix is the Galois group. In the general case, i.e., in the presence of an irregular singularity, the monodromy is no longer sufficient to provide a complete collection of topological generators. Ramis has shown that the group generated by the monodromy, the exponential torus and the Stokes operators, which is defined in a transcendental way as a subgroup of the differential Galois group, is dense in the latter in the Zariski topology. 
More recently, a Galois theory for parametrized linear differential equations of the form

$$
\partial_{z} Y(z, t)=A(z, t) Y(z, t)
$$

where $t=\left(t_{1}, \ldots, t_{n}\right)$ are parameters and $A$ is a matrix whose entries lie in a certain field (specified explicitly throughout), has been developed in [Cassidy and Singer 2007] (henceforth abbreviated [CS]); see also [Hardouin and Singer 2008; Landesman 2008; Robinson 1959; Umemura 1996]. Namely, the Galois group, which measures the $\left(\partial_{t_{1}}, \ldots, \partial_{t_{n}}\right)$-differential and algebraic dependencies among the solutions, can be seen as a differential group in the sense of Kolchin, that is, a group of matrices whose entries lie in a differential field and satisfy a set of polynomial differential equations in the variables $t_{1}, \ldots, t_{n}$; see [Cassidy 1972; 1989; Kolchin 1973; 1985; Minchenko and Ovchinnikov 2011]. The theory from [CS] requires the field of constants with respect to $\partial_{z}$ to be of characteristic 0 and differentially closed (see Section 2A). The drawback of this latter assumption is that a differentially closed field is a very big field, and cannot be interpreted as a field of functions.

There is a link between the parametrized differential Galois theory and isomonodromy for equations with only regular singular poles (see [Cassidy and Singer 2007; Mitschi and Singer 2012; 2013]. Let

$$
\mathscr{D}\left(t_{0}, r\right)=\left\{\left(z_{1}, \ldots, z_{n}\right) \in \mathbb{C}^{n}|| z_{i}-t_{0, i} \mid<r \text { for all } i \leq n\right\}
$$

be an open polydisc in $\mathbb{C}^{n}$, let $\mathscr{D}$ be an open subset of $\mathbb{C}$, and let $A(z, t)$ be a matrix whose entries are analytic on $\mathscr{D} \times \mathscr{D}\left(t_{0}, r\right)$. We consider open disks $D_{j}$ that cover $\mathscr{D}$, and solutions $U_{j}(z, t)$ of $(*)$ that are analytic on $D_{j} \times \mathscr{D}\left(t_{0}, r\right)$. If $D_{i} \cap D_{j} \neq \varnothing$, we define the connection matrices $C_{i, j}(t)=U_{i}(z, t)^{-1} U_{j}(z, t)$. Following Definition 5.2 in [CS] (see also [Bolibruch 1997; Malgrange 1983]), the parametrized linear differential equation $(*)$ is said to be isomonodromic if there is a choice of $\left(D_{i}\right)$ covering $\mathscr{D}$ and of the solutions $U_{i}(z, t)$ of $(*)$, analytic on $D_{i} \times \mathscr{D}\left(t_{0}, r\right)$, such that the connection matrices are independent of $t$. In this case, the matrix of the monodromy is constant on the polydisc $\mathscr{D}\left(t_{0}, r\right)$. When $A(z, t)$ is of the form $\sum_{i=1}^{s} A_{i}(t) /\left(z-u_{i}\right)$ such that all the $A_{i}(t)$ have analytic entries on $U$ and $u_{i} \in \mathscr{D}$, the following statements are equivalent (see [CS], Propositions 5.3 and 5.4):

- The Galois group is conjugate over a differentially closed field (Definition 2.2) to a group of constant matrices.

- The parametrized linear differential equation is isomonodromic in the above sense. 
- The parametrized linear differential equation is completely integrable (see Definition 3.1).

We are interested in the case where the parametrized linear differential equation may have irregular singularities, in a sense we are going to explain. The main result of this paper is a parametrized analogue of the density theorem of Ramis: we give topological generators for the Galois group in the Kolchin topology (in which closed sets are zero sets of differential algebraic polynomials). As an application of our main result, we improve Proposition 3.9 in [CS] (see Remark 3.4): a parametrized linear differential equation is completely integrable if and only if the topological generators for the Galois group just mentioned are conjugate to constant matrices over a field of meromorphic functions. Notice that the latter is not differentially closed.

The article is organized as follows. In the first section we study parametrized linear differential systems from an analytic point of view. The parameters will vary in $U$, a nonempty polydisc in $\mathbb{C}^{n}$. Let $t=\left(t_{1}, \ldots, t_{n}\right) \in U$ denote the multiparameter. Let $M_{U}$ be the field of meromorphic functions on $U$ and let $\hat{K}_{U}=M_{U} \llbracket z \rrbracket\left[z^{-1}\right]$. The Hukuhara-Turrittin theorem in this case gives the following result (see Remark 1.6 for a discussion of a similar result present in [Schäfke 2001]):

Proposition 1.3. Consider the equation $\partial_{z} Y(z, t)=A(z, t) Y(z, t)$, with $A(z, t) \in$ $\mathrm{M}_{m}\left(\hat{K}_{U}\right)$ (that is, an $m \times m$ matrix with entries in $\left.\hat{K}_{U}\right)$. Then there exist a nonempty polydisc $U^{\prime} \subset U$ and $v \in \mathbb{N}^{*}$ such that we have a fundamental solution $F(z, t)$ of the form

$$
F(z, t)=\hat{H}(z, t) z^{L(t)} e^{Q(z, t)},
$$

where:

- $\hat{H}(z, t) \in \mathrm{GL}_{m}\left(\hat{K}_{U^{\prime}}\left[z^{1 / \nu}\right]\right)$.

- $L(t) \in \mathrm{M}_{m}\left(M_{U^{\prime}}\right)$.

- $e^{Q(z, t)}=\operatorname{Diag}\left(e^{q_{i}(z, t)}\right)$, with $q_{i}(z, t) \in z^{-1 / v} M_{U^{\prime}}\left[z^{-1 / v}\right]$.

- Moreover, we have $z^{L(t)} e^{Q(z, t)}=e^{Q(z, t)} z^{L(t)}$.

See Remark 1.4 for a discussion about the uniqueness of a fundamental solution of $(*)$ written in this way.

In Section 1C, we briefly review the Stokes phenomenon in the unparametrized case. We have solutions that are analytic in some sector and Gevrey asymptotic to the formal part of the solution in the Hukuhara-Turrittin canonical form. The fact that various asymptotic solutions do not glue to a single solution on the Riemann surface of the logarithm is called the Stokes phenomenon.

Let $U$ be a nonempty polydisc in $\mathbb{C}^{n}$ and let $f(z, t)=\sum f_{i}(t) z^{i} \in \hat{K}_{U}$. We say that $f(z, t)$ belongs to $\mathrm{O}_{U}(\{z\})$ if for all $t \in U, z \mapsto \sum f_{i}(t) z^{i}$ is the germ of a 
meromorphic function at 0 . Remark that if

$$
f(z, t) \in \hat{O}_{U}(\{z\}) \subset M_{U} \llbracket z \rrbracket\left[z^{-1}\right]=\hat{K}_{U},
$$

then the $z$-coefficients $f_{i}(t)$ of $f(z, t)$ are analytic on $U$.

In Section 1D, we study the Stokes phenomenon for equations of the form $(*)$ with $A(z, t) \in \mathrm{M}_{m}\left(\mathscr{O}_{U}(\{z\})\right)$. In particular, we prove that the asymptotic solutions depend analytically (under mild conditions) upon the parameters.

In the second section, we use the parametrized Hukuhara-Turrittin theorem to deduce some Galois-theoretic properties of parametrized linear differential equations in coefficients in $\mathrm{O}_{U}(\{z\})$. We first recall some facts from [CS] about parametrized differential Galois theory. The problem is that the theory in this reference cannot be applied here, since $M_{U}$, our field of constants with respect to $\partial_{z}$, is a field of functions that are meromorphic in $t_{1}, \ldots, t_{n}$, and this field is not differentially closed (see Section 2A). In the papers [Gillet et al. 2013; Wibmer 2012], the authors prove the existence of parametrized Picard-Vessiot extensions under weaker assumptions than in [CS]. See also [Chatzidakis et al. 2008; Peón Nieto 2011]. We do not use these latter results because we need a parametrized Hukuhara-Turrittin theorem (which proves directly that a parametrized Picard-Vessiot extension exists, not necessarily unique) in order to study the parametrized Stokes phenomenon. This allow us to define a group that we will call, by abuse of language, the parametrized differential Galois group; see Remark 2.8. In Section 2D we consider the local case of (*), with $A(z, t) \in \mathrm{M}_{m}\left(\mathscr{O}_{U}(\{z\})\right)$. We state and show the main result:

Theorem 2.20 (parametrized analogue of the density theorem of Ramis). The group generated by the parametrized monodromy, the parametrized exponential torus and the parametrized Stokes operators is dense in the parametrized differential Galois group for the Kolchin topology.

Then, we turn to the global case. We consider equations with coefficients in $M_{U}(z)$ and study their global Galois group. We prove a density theorem in this global setting; see Theorem 2.24. The proof in the unparametrized case can be found in [Mitschi 1996]. In Section 2F, we give various examples of calculations.

In the third section, we give three applications. First, we prove a criterion for the integrability of differential systems (see Definition 3.1):

Proposition 3.2. Let $A(z, t) \in \mathrm{M}_{m}\left(M_{U}(z)\right)$. Then the linear differential equation $\partial_{z} Y(z, t)=A(z, t) Y(z, t)$ is completely integrable if and only if there exists a fundamental solution such that the matrices of the parametrized monodromy, the parametrized exponential torus and the parametrized Stokes operators for all the singularities are constant, i.e., do not depend on $z$.

As a second application, we give a partial answer to a question of Sibuya [1975] regarding the differential transcendence properties of a Stokes matrix of the 
parametrized linear differential equation

$$
\left(\begin{array}{c}
\partial_{z} Y(z, t) \\
\partial_{z}^{2} Y(z, t)
\end{array}\right)=\left(\begin{array}{cc}
0 & 1 \\
z^{3}+t & 0
\end{array}\right)\left(\begin{array}{c}
Y(z, t) \\
\partial_{z} Y(z, t)
\end{array}\right)
$$

Sibuya was asking whether an entry of a given Stokes matrix at infinity is $\partial_{t^{-}}$ differentially transcendental, i.e., satisfies no differential polynomial equation. We prove that it is at least not $\partial_{t}$-finite, i.e., that it satisfies no linear differential equation.

As a last application, we deal with the inverse problem. We prove that if $G$ is the global parametrized differential Galois group of some equation having coefficients in $k(z)$ (see Section 3C), then $G$ contains a finitely generated Kolchin-dense subgroup. The converse of this latter assertion has been proved in Corollary 5.2 of [Mitschi and Singer 2012], and we obtain a result on the inverse problem:

Theorem 3.11. $G$ is the global parametrized differential Galois group of some equation having coefficients in $k(z)$ if and only if $G$ contains a finitely generated Kolchin-dense subgroup.

In the Appendix, we prove the following result:

Theorem A.1. Consider the equation $\partial_{z} Y(z, t)=A(z, t) Y(z, t)$, with $A(z, t) \in$ $\mathrm{M}_{m}\left(\hat{K}_{U}\right)$. Then there exists a nonempty polydisc $U^{\prime} \subset U$ such that we have a fundamental solution $F(z, t)$ of the form

$$
F(z, t)=\hat{P}(z, t) z^{C(t)} e^{Q(z, t)},
$$

where:

- $\hat{P}(z, t) \in \mathrm{GL}_{m}\left(\hat{K}_{U^{\prime}}\right)$,

- $C(t) \in \mathrm{M}_{m}\left(M_{U^{\prime}}\right)$,

- $e^{Q(z, t)}=\operatorname{Diag}\left(e^{q_{i}(z, t)}\right)$, with $q_{i}(z, t) \in z^{-1 / v} \mathcal{M}_{U^{\prime}}\left[z^{-1 / v}\right]$, for some $v \in \mathbb{N}^{*}$.

Remark that contrary to Proposition 1.3, the entries of the formal part are not ramified. On the other hand, $z^{C(t)}$ and $e^{Q(z, t)}$ do not commute anymore. This theorem is not necessary for the proof of the main result of the paper; this is the reason why we give the proof in the Appendix. However, this result is important since it permits one to determine the equivalence classes (see [van der Put and Singer 2003, p. 7]) of parametrized linear differential systems in coefficients in $\hat{K}_{U}$.

\section{Local analytic linear differential systems depending upon parameters}

In Section 1A, we define the field to which the entries of the fundamental solution, in the Hukuhara-Turrittin canonical form, will belong. In Section 1B, we prove a parametrized version of the Hukuhara-Turrittin theorem. In Section 1C, we briefly review the Stokes phenomenon in the unparametrized case. In Section 1D, we study the Stokes phenomenon in the parametrized case. 
1A. Definition of the fields. Let us consider a linear differential system of the form $\partial_{z} Y(z)=A(z) Y(z)$, where $A(z)$ is an $m \times m$ matrix whose entries belongs to $\mathbb{C} \llbracket z \rrbracket\left[z^{-1}\right]$. We know we can find a formal fundamental solution in the HukuharaTurrittin canonical form $\hat{H}(z) z^{L} e^{Q(z)}$, where:

- $\hat{H}(z)$ is a matrix of formal power series in $z^{1 / v}$ for some $v \in \mathbb{N}^{*}$.

- $L \in \mathrm{M}_{m}(\mathbb{C})$.

- $Q(z)=\operatorname{Diag}\left(q_{i}(z)\right)$, with $q_{i}(z) \in z^{-1 / v} \mathbb{C}\left[z^{-1 / v}\right]$.

- Moreover, we have $z^{L} e^{Q(z)}=e^{Q(z)} z^{L}$.

Notice that this formulation is trivially equivalent to Theorem 3.1 in [van der Put and Singer 2003]. Let $U$ be a nonempty polydisc of $\mathbb{C}^{n}$, and define $\hat{K}_{U}$ and $\mathcal{M}_{U}$ as on page 90 . We want to construct a field containing a fundamental set of solutions of $(*)$, where $A(z, t) \in \mathrm{M}_{m}\left(\hat{K}_{U}\right)$. Let $\Delta_{t}=\left\{\partial_{t_{1}}, \ldots, \partial_{t_{n}}\right\}$ and let

$$
\mathbf{E}_{U}=\bigcup_{\nu \in \mathbb{N}^{*}} z^{-1 / \nu} M_{U}\left[z^{-1 / \nu}\right]
$$

We define formally the $\left(\partial_{z}, \Delta_{t}\right)$-ring, i.e., a ring equipped with $n+1$ derivations $\partial_{z}, \partial_{t_{1}}, \ldots, \partial_{t_{n}}$, a priori not required to commute with each other, to be

$$
R_{U}:=\hat{K}_{U}\left[\log ,\left(z^{a(t)}\right)_{a(t) \in \mathcal{M}_{U}}, l(e(q(z, t)))_{q(z, t) \in \mathbf{E}_{U}}\right],
$$

with the following rules:

(1) The symbols $\log ,\left(z^{a(t)}\right)_{a(t) \in \mathcal{M}_{U}}$ and $(e(q(z, t)))_{q(z, t) \in \mathbf{E}_{U}}$ only satisfy the following relations:

$$
\begin{gathered}
z^{a(t)+b(t)}=z^{a(t)} z^{b(t)}, \quad z^{a}=z^{a} \in \hat{K}_{U} \quad \text { for } a \in \mathbb{Z}, \\
e\left(q_{1}(z, t)+q_{2}(z, t)\right)=e\left(q_{1}(z, t)\right) e\left(q_{2}(z, t)\right), \quad e(0)=1 .
\end{gathered}
$$

(2) The following rules of differentiation:

$$
\begin{gathered}
\partial_{z} \log =z^{-1}, \quad \partial_{t_{i}} \log =0, \quad \partial_{z} z^{a(t)}=\frac{a(t)}{z} z^{a(t)}, \quad \partial_{t_{i}} z^{a(t)}=\partial_{t_{i}}(a(t)) \log z^{a(t)}, \\
\partial_{z} e(q(z, t))=\partial_{z}(q(z, t)) e(q(z, t)), \quad \partial_{t_{i}} e(q(z, t))=\partial_{t_{i}}(q(z, t)) e(q(z, t)),
\end{gathered}
$$

equip the ring with a $\left(\partial_{z}, \Delta_{t}\right)$-differential structure, since these rules descend to the quotient, as can be readily checked.

The intuitive interpretations of these symbols are: $\log =\log (z), z^{a(t)}=e^{a(t) \log (z)}$ and $e(q(z, t))=e^{q(z, t)}$. Let $f(z, t)$ be one these latter functions. Then $f(z, t)$ has a natural interpretation as an analytic function on $\widetilde{\mathbb{C}} \times U^{\prime}$, where $\widetilde{\mathbb{C}}$ is the Riemann surface of the logarithm and $U^{\prime}$ is some nonempty polydisc contained in $U$. We will use the analytic function instead of the symbol when we will consider asymptotic 
solutions (see Section 1C and Section 1D). For the time being, however, we see them only as symbols.

Let $\bar{M}_{U}$ be the algebraic closure of $\mathcal{M}_{U}$. In the same way as for $R_{U}$, we construct the $\left(\partial_{z}, \Delta_{t}\right)$-ring

$$
\bar{R}_{U}:=\bar{M}_{U} \llbracket z \rrbracket\left[z^{-1}\right]\left[\log ,\left(z^{a(t)}\right)_{a(t) \in \overline{\mathcal{M}}_{U}},(e(q(z, t)))_{q(z, t) \in \mathscr{E}_{U}}\right],
$$

where

$$
\mathscr{\mathscr { E }}_{U}=\bigcup_{\nu \in \mathbb{N}^{*}} z^{-1 / \nu} \bar{M}_{U}\left[z^{-1 / \nu}\right]
$$

and its field of fractions has field of constants with respect to $\partial_{z}$ equal to $\bar{M}_{U}$. Since $R_{U} \subset \bar{R}_{U}, R_{U}$ is also an integral domain. Therefore, we may consider the $\left(\partial_{z}, \Delta_{t}\right)$-fields

$$
\begin{aligned}
& K_{F, U}=\mathcal{M}_{U}\left(\log ,\left(z^{a(t)}\right)_{a(t) \in \mathcal{M}_{U}}\right), \\
& \hat{K}_{F, U}=\hat{K}_{U}\left(\log ,\left(z^{a(t)}\right)_{a(t) \in \mathcal{M}_{U}}\right),
\end{aligned}
$$

and

$$
\left(\mathbf{K}_{U}\right)^{\wedge}=\hat{K}_{U}\left(\log ,\left(z^{a(t)}\right)_{a(t) \in \mathcal{M}_{U}},(e(q(z, t)))_{q(z, t) \in \mathbf{E}_{U}}\right) .
$$

In the definition of the fields $K_{F, U}$ and $\hat{K}_{F, U}$, the subscript $F$ stands for Fuchsian. Since $\left(\mathbf{K}_{U}\right)^{\wedge}$ is contained in the field of fractions of $\bar{R}_{U}$, its field of constants with respect to $\partial_{z}$ is equal to $\bar{M}_{U} \cap\left(\mathbf{K}_{U}\right)^{\wedge}=\mu_{U}$.

We have defined $\left(\partial_{z}, \Delta_{t}\right)$-fields where all the derivations commute with each other. We have the following inclusions of $\left(\partial_{z}, \Delta_{t}\right)$-fields:

$$
\begin{aligned}
\nearrow^{K_{F, U}} & \\
\mu_{U} \rightarrow \hat{K}_{U} & \rightarrow \hat{K}_{F, U} \rightarrow\left(\mathbf{K}_{U}\right)^{\wedge} .
\end{aligned}
$$

Remark 1.1. Any algebraic function over $\mu_{U}$ can be seen as an element of $\mu_{U^{\prime}}$ for some nonempty $U^{\prime} \subset U$. Therefore, a finite extension of $\mu_{U}$ can be embedded in $M_{U^{\prime}}$ for a convenient choice of $U^{\prime} \subset U$. We will use this fact in the rest of the paper.

Lemma 1.2. Let $U \subseteq \mathbb{C}^{n}$ be a nonempty polydisc, and let $L(t) \in \mathrm{M}_{m}\left(\bar{M}_{U}\right)$, where $\bar{M}_{U}$ is the algebraic closure of $M_{U}$. There exist a nonempty polydisc $U^{\prime} \subset U$ and $z^{L(t)} \in \mathrm{GL}_{m}\left(K_{F, U^{\prime}}\right)$ satisfying

$$
\partial_{z} z^{L(t)}=\frac{L(t)}{z} z^{L(t)}=z^{L(t)} \frac{L(t)}{z} .
$$

Proof. Let

$$
L(t)=P(t)(D(t)+N(t)) P^{-1}(t)
$$


be the Jordan decomposition of $L(t)$, where $D(t)=\operatorname{Diag}\left(d_{i}(t)\right)$ with $d_{i}(t) \in \bar{M}_{U}$, $N(t)$ is nilpotent, $D(t) N(t)=N(t) D(t)$ and $P(t) \in \mathrm{GL}_{m}\left(\bar{M}_{U}\right)$.

Due to Remark 1.1, there exists a nonempty polydisc $U^{\prime} \subset U$ such that $d_{i}(t) \in M_{U^{\prime}}$ and $P(t) \in \mathrm{GL}_{m}\left(M_{U^{\prime}}\right)$. We may restrict $U^{\prime}$ and assume that $N(t)$ does not depend upon $t$ in $U^{\prime}$. Let us write $N:=N(t)$. Then the matrix

$$
z^{L(t)}=P(t) \operatorname{Diag}\left(z^{d_{i}(t)}\right) e^{N \log } P^{-1}(t)
$$

belongs to $\mathrm{GL}_{m}\left(K_{F, U^{\prime}}\right)$, and $z^{L(t)}$ satisfies

$$
\partial_{z} z^{L(t)}=\frac{L(t)}{z} z^{L(t)}=z^{L(t)} \frac{L(t)}{z} .
$$

Let $a(t) \in M_{U}$ and let $(a(t)) \in \mathrm{M}_{1}\left(M_{U}\right)$ be the corresponding matrix. Then we have $z^{a(t)}=z^{(a(t))}$.

1B. The Hukuhara-Turrittin theorem in the parametrized case. The goal of this subsection is to give the parametrized version of the Hukuhara-Turrittin theorem. In the Appendix, we prove a slightly different result, which is not needed in the paper; see Theorem A.1.

Proposition 1.3. Let $U$ be a nonempty polydisc in $\mathbb{C}^{n}$ and consider the equation

$$
\partial_{z} Y(z, t)=A(z, t) Y(z, t),
$$

with $A(z, t) \in \mathrm{M}_{m}\left(\hat{K}_{U}\right)$. There exists a nonempty polydisc $U^{\prime} \subset U$ such that we have a fundamental solution $F(z, t) \in \mathrm{GL}_{m}\left(\left(\mathbf{K}_{U^{\prime}}\right)^{\wedge}\right)$ of the form

$$
F(z, t)=\hat{H}(z, t) z^{L(t)} e(Q(z, t)),
$$

where:

- $\hat{H}(z, t) \in \mathrm{GL}_{m}\left(\hat{K}_{U^{\prime}}\left[z^{1 / \nu}\right]\right)$, for some $v \in \mathbb{N}^{*}$.

- $L(t) \in \mathrm{M}_{m}\left(M_{U^{\prime}}\right)$.

- $e(Q(z, t))=\operatorname{Diag}\left(e\left(q_{i}(z, t)\right)\right)$, with $q_{i}(z, t) \in \mathbf{E}_{U^{\prime}}$.

- Moreover, we have e $(Q(z, t)) z^{L(t)}=z^{L(t)} e(Q(z, t))$.

Furthermore, if $A(z, t) \in \mathrm{M}_{m}\left(\mathcal{O}_{U}(\{z\})\right)$, there exists a nonempty polydisc $U^{\prime \prime} \subset U^{\prime}$ such that we may assume that the z-coefficients of $\hat{H}(z, t)$ are all analytic on $U^{\prime \prime}$.

Remark 1.4. Remark that we have no uniqueness of the fundamental solution written in this way, since $z^{\kappa} \hat{H}(z, t) z^{L(t)-\kappa} e^{Q(z, t)}$ is also a fundamental solution for all $\kappa \in \mathbb{Z}$. However, by the construction of $\left(\mathbf{K}_{U^{\prime}}\right)^{\wedge}$, if $\hat{H}_{i}(z, t) z^{L_{i}(t)} e\left(Q_{i}(z, t)\right)$ are fundamental solutions of $(*)$ written in this way for $i=1,2$, then, up to a permutation, $Q_{1}$ and $Q_{2}$ have the same entries. 
Example 1.5 [Schäfke 2001, Introduction]. If we consider

$$
z^{2} \partial_{z} Y(z, t)=\left(\begin{array}{ll}
t & 1 \\
z & 0
\end{array}\right) Y(z, t)
$$

we get the solution

$$
\left(\left(\begin{array}{cc}
1 & 1 \\
0 & -t
\end{array}\right)+O(z)\right)\left(\begin{array}{cc}
z^{1 / t} e^{-t / z} & 0 \\
0 & z^{-1 / t}
\end{array}\right)
$$

for $t \neq 0$, and the solution

$$
\left(\begin{array}{cc}
1 & 1 \\
z^{1 / 2} & -z^{1 / 2}
\end{array}\right)\left(\left(\begin{array}{ll}
1 & 0 \\
0 & 1
\end{array}\right)+O\left(z^{1 / 2}\right)\right)\left(\begin{array}{cc}
z^{1 / 4} e^{-z^{-1 / 2}} & 0 \\
0 & z^{1 / 4} e^{z^{-1 / 2}}
\end{array}\right)
$$

for $t=0$. The latter is not the specialization of (1-1) at $t=0$. The problem is that the level of the unparametrized system (see Section $1 \mathrm{C}$ for the definition) at $t=0$ is 1 and the level of the unparametrized system for $t \neq 0$ is $\frac{1}{2}$. This example shows that we cannot get a solution in the parametrized Hukuhara-Turrittin form that remains valid for all values of the parameter $t$. This is the reason why we have to restrict the subset of the parameter space.

Remark 1.6. Similar results to Proposition 1.3 have been proved in Theorem 4.2 of [Schäfke 2001]. We now explain the result of Schäfke. Let $U$ be an open connected subset of $\mathbb{C}^{n}$ that contains 0 , and let $A(z, t)=\sum_{l=s}^{\infty} A_{l}(t)$, with $s \in \mathbb{Z}$ and $A_{l}(t)$ analytic in $U$. In particular, $A(z, t) \in \mathrm{M}_{m}\left(\hat{K}_{U}\right)$. Assume that, for all $t \in U$, there exists a solution $\hat{H}_{t}(z) z^{L_{t}} e(Q(z, t))$ to $(*)$ given in the classical Hukuhara-Turrittin canonical form, i.e., such that:

- The $z$-coefficients of the $q_{i}(z, t)$ are analytic functions in $t \in U$.

- The degree in $z^{-1}$ of $q_{i}(z, t)-q_{j}(z, t)$ is independent of $t$ in $U$.

- If $q_{i}(z, t) \not \equiv q_{j}(z, t)$, then $q_{i}(z, 0) \neq q_{0}(z, 0)$.

Under these assumptions, Schäfke concludes that there exists an open neighborhood $U^{\prime} \subset U$ of 0 in the $t$-plane such that there exists a solution

$$
\hat{H}(z, t) z^{L(t)} e(Q(z, t)) \in \mathrm{GL}_{m}\left(\left(\mathbf{K}_{U^{\prime}}\right)^{\wedge}\right)
$$

with $\hat{H}(z, t)=\sum_{l=0}^{\infty} \hat{H}_{l}(t)$ and such that the maps $t \mapsto \hat{H}_{l}(t), L(t)$ are analytic. Notice that Schäfke gives a necessary and sufficient condition, that can be algorithmically checked, for well-behaved exponential part. See [ibid., Theorem 5.2]. Using Schäfke's theorem, we can deduce Proposition 1.3 only in the particular case where $A(z, t)$ has entries with $z$-coefficients analytic in $U$. Note that [ibid.] does not allow us to deduce the general case. See also [Babbitt and Varadarajan 1985, $\S 10$, Theorem 1] for another result of this nature. 
Proof of Proposition 1.3. Let $K=C \llbracket z \rrbracket\left[z^{-1}\right]$, where $C$ is an algebraically closed field of characteristic 0 , equipped with a derivation $\partial_{z}$ that acts trivially on $C$ and with $\partial_{z}(z)=1$. The Hukuhara-Turrittin theorem (see Theorem 3.1 in [van der Put and Singer 2003]) is valid for linear differential system with entries in $K$. We apply it with $C=\bar{M}_{U}$, the algebraic closure of $\mu_{U}$.

Let us consider the matrices $L(t) \in \mathrm{M}_{m}\left(\bar{M}_{U}\right)$ and $Q(z, t)=\operatorname{Diag}\left(q_{i}(z, t)\right)$, with $q_{i}(z, t) \in z^{-1 / v} \overline{\mathcal{M}}_{U}\left[z^{-1 / \nu}\right]$ for some $v \in \mathbb{N}$. Because of Remark 1.1 and Lemma 1.2, there exists a nonempty polydisc $U^{\prime} \subset U$ such that we may define $z^{L(t)} \in \mathrm{GL}_{m}\left(K_{F, U^{\prime}}\right)$ satisfying

$$
\partial_{z} z^{L(t)}=\frac{L(t)}{z} z^{L(t)}=z^{L(t)} \frac{L(t)}{z},
$$

$L(t) \in \mathrm{M}_{m}\left(\mathcal{M}_{U^{\prime}}\right)$ and $q_{i}(z, t) \in \mathbf{E}_{U^{\prime}}$. Hence, the Hukuhara-Turrittin theorem gives a fundamental solution

$$
F^{\prime}(z, t)=\hat{H}^{\prime}(z, t) z^{L(t)} e(Q(z, t))
$$

on $U^{\prime}$, where:

- $\hat{H}^{\prime}(z, t) \in \mathrm{GL}_{m}\left(\overline{\mathcal{M}}_{U^{\prime}} \llbracket z^{1 / v} \rrbracket\left[z^{-1 / \nu}\right]\right)$, for some $v \in \mathbb{N}$.

- $L(t) \in \mathrm{M}_{m}\left(M_{U^{\prime}}\right)$.

- $e(Q(z, t))=\operatorname{Diag}\left(e\left(q_{i}(z, t)\right)\right)$, with $q_{i}(z, t) \in \mathbf{E}_{U^{\prime}}$.

- Moreover, we have $e(Q(z, t)) z^{L(t)}=z^{L(t)} e(Q(z, t))$.

Let us prove now that we may find $\hat{H}(z, t) \in \mathrm{GL}_{m}\left(\hat{K}_{U^{\prime}}\left[z^{1 / \nu}\right]\right)$ such that

$$
F(z, t)=\hat{H}(z, t) z^{L(t)} e(Q(z, t))
$$

is a fundamental solution. The matrix

$$
F^{\prime}(z, t)=\hat{H}^{\prime}(z, t) z^{L(t)} e(Q(z, t))
$$

satisfies the parametrized linear differential equation

$$
\partial_{z} F^{\prime}(z, t)=A(z, t) F^{\prime}(z, t),
$$

and the matrix $z^{L(t)} e(Q(z, t))$ satisfies the parametrized linear differential equation

$$
\begin{aligned}
\partial_{z} z^{L(t)} e(Q(z, t)) & =\left(z^{-1} L(t)+\partial_{z} Q(z, t)\right) z^{L(t)} e(Q(z, t)) \\
& =z^{L(t)} e(Q(z, t))\left(z^{-1} L(t)+\partial_{z} Q(z, t)\right) .
\end{aligned}
$$

Hence

$$
\partial_{z} \hat{H}^{\prime}(z, t)=A(z, t) \hat{H}^{\prime}(z, t)-\hat{H}^{\prime}(z, t)\left(z^{-1} L(t)+\partial_{z} Q(z, t)\right) .
$$

We write $\hat{H}^{\prime}(z, t)$ as a column vector $\widetilde{H}^{\prime}(z, t)$ of size $m^{2}$. Let

$$
C(z, t) \in \mathrm{M}_{m^{2}}\left(\hat{K}_{U^{\prime}}\left[z^{1 / \nu}\right]\right),
$$


with $v \in \mathbb{N}^{*}$ such that $\widetilde{H}^{\prime}(z, t)$ satisfies the parametrized linear differential system

$$
\partial_{z} \widetilde{H}^{\prime}(z, t)=C(z, t) \widetilde{H}^{\prime}(z, t) .
$$

Let us write

$$
\tilde{H}^{\prime}(z, t)=\sum_{i \geq N} \tilde{H}_{i}^{\prime}(t) z^{i / v} \quad \text { and } \quad C(z, t)=\sum_{i \geq M} C_{i}(t) z^{i / v}
$$

where $M, N \in \mathbb{Z}$. Then, by identifying the coefficients of the $z^{i / v}$-terms of the power series in the equation $\partial_{z} \widetilde{H}^{\prime}(z, t)=C(z, t) \widetilde{H}^{\prime}(z, t)$, we find that

$$
\left(\frac{i}{v}+1\right) \tilde{H}_{i+v}^{\prime}(t)=\sum_{l=N}^{i-M} C_{i-l}(t) \tilde{H}_{l}^{\prime}(t) .
$$

By the definition of $\hat{K}_{U^{\prime}}\left[z^{1 / \nu}\right]$, every $C_{i}(t)$ belongs to $\mathrm{M}_{m}\left(\mathcal{M}_{U^{\prime}}\right)$. The fact that there exists a fundamental solution $\hat{H}(z, t) z^{L(t)} e(Q(z, t))$ with $\hat{H}(z, t) \in \operatorname{GL}_{m}\left(\hat{K}_{U^{\prime}}\left[z^{1 / \nu}\right]\right)$ is now clear.

Assume now that $A(z, t) \in \mathrm{M}_{m}\left(\mathscr{O}_{U}(\{z\})\right)$. Let $U^{\prime \prime}$ be a nonempty polydisc with $U^{\prime \prime} \subset U^{\prime}$ such that for $z \neq 0$ fixed, the entries of the $z$-coefficients of

$$
z^{-1} L(t)+\partial_{z} Q(z, t)
$$

are analytic on $U^{\prime \prime}$. Then the entries of the $z$-coefficients of $C(z, t)$ are all analytic on $U^{\prime \prime}$. Hence, we may assume that the entries of the $z$-coefficients of $\hat{H}(z, t)$ are all analytic on $U^{\prime \prime}$.

Remark 1.7. If we take a smaller nonempty polydisc $U$, we may assume that if we consider the equation (*) with $A(z, t) \in \mathrm{M}_{m}\left(\mathcal{O}_{U}(\{z\})\right)$, then the fundamental solution of Proposition 1.3 belongs to $\mathrm{GL}_{m}\left(\left(\mathbf{K}_{U}\right)^{\wedge}\right)$, and the entries of the $z$-coefficients of $\hat{H}(z, t)$ are all analytic on $U$.

1C. Review of the Stokes phenomenon in the unparametrized case. In this subsection we will briefly review the Stokes phenomenon in the unparametrized case. See [Cano and Ramis 1995; Écalle 1981; Loday-Richaud 1990; 1994; 1995; LodayRichaud and Remy 2011; Malgrange 1991; 1995; Malgrange and Ramis 1992; Ramis 1980; 1985; Rasoamanana 2010; Remy 2012; Ramis and Sibuya 1989; Singer 2009; Wasow 1965], and in particular Chapter 8 of [van der Put and Singer 2003] for more details. We will generalize some results concerning the summation of divergent series in the parametrized case in Section 1D. First we treat the example of the Euler equation

$$
z^{2} \partial_{z} Y(z)+Y(z)=z
$$


which admits as a solution the formal series $\hat{f}(z)=\sum_{n=0}^{\infty}(-1)^{n} n ! z^{n+1}$. Classical methods of differential equations give another solution:

$$
f(z)=\int_{0}^{z} e^{1 / z} e^{-1 / t} \frac{d t}{t}=\int_{0}^{\infty} \frac{1}{1+u} e^{-u / z} d u
$$

where $1 / t-1 / z=u / z$. The solution $\hat{f}(z)$ is divergent and the solution $f(z)$ can be extended to an analytic function on the sector $V=\Sigma(-3 \pi / 2,3 \pi / 2)$, where, here and throughout, we use the notation

$$
\Sigma(\alpha, \beta):=\{z \in \widetilde{\mathbb{C}} \mid \arg (z) \in] \alpha, \beta[\}
$$

to represent sectors in $\widetilde{\mathbb{C}}$. On this sector, $f(z)$ is 1 -Gevrey asymptotic to $\hat{f}(z)$ : for every closed subsector $W$ of $V$, there exist $A_{W} \in \mathbb{R}$ and $\varepsilon>0$ such that for all $N$ and all $z \in W$ with $|z|<\varepsilon$,

$$
\left|f(z)-\sum_{n=0}^{N-1}(-1)^{n} n ! z^{n+1}\right| \leq\left(A_{W}\right)^{N+1}(N+1) !|z|^{N+1} .
$$

We can also consider $f\left(e^{2 i \pi} z\right)$, which is an asymptotic solution on the sector

$$
V^{\prime}=\Sigma(\pi / 2,7 \pi / 2) \text {. }
$$

The two asymptotic solutions do not glue to a single asymptotic solution on $V \cup V^{\prime}$. In fact, the residue theorem implies that the difference in $V \cap V^{\prime}$ of the two asymptotic solutions is

$$
2 i \pi e^{1 / z}
$$

The fact that various asymptotic solutions do not glue to a single analytic solution is called the Stokes phenomenon.

More generally, let us consider a linear differential equation $\partial_{z} Y(z)=A(z) Y(z)$ such that the entries of $A(z)$ are germs of meromorphic functions in a neighborhood of 0 . Let $\hat{H}(z) z^{L} e(Q(z))$ be a fundamental solution in the Hukuhara-Turrittin canonical form, with $Q(z)=\operatorname{Diag}\left(q_{i}(z)\right)$. Since

$$
\hat{H}(z) z^{L} e(Q(z))=\hat{H}(z) \operatorname{Diag}\left(z^{k}\right) z^{L-k \operatorname{Id}} e(Q(z))
$$

for all $k \in \mathbb{N}$, we may assume that $\hat{H}(z)$ has no pole at $z=0$. The levels of $\partial_{z} Y(z)=A(z) Y(z)$ are the degrees in $z^{-1}$ of the $q_{i}(z)-q_{j}(z)$ (the levels are positive rational numbers and are well-defined because of Remark 1.4). Consider

$$
q(z)=q_{k} z^{-k / v}+\cdots+q_{1} z^{-1 / v} \in z^{-1 / v} \mathbb{C}\left[z^{-1 / v}\right]
$$

with $v \in \mathbb{N}$. The real number $d$ is called singular for $q(z)$ if $q_{k} e^{-i d k / v}$ is a positive real number. These correspond to the arguments $d$ such that $r \mapsto e^{q\left(r e^{i d}\right)}$ increases fastest as $r$ tends to $0^{+}$. The singular directions of $\partial_{z} Y(z)=A(z) Y(z)$ (we will 
write singular directions when no confusion is likely to arise) are the real numbers that are singular for one of the $q_{i}(z)-q_{j}(z)$, with $i \neq j$. Notice that the set of singular directions is finite modulo $2 \pi v$ for some $v \in \mathbb{N}$. Let $k_{1}<\cdots<k_{r}$ be the levels of the linear differential equation. There exists a decomposition $\hat{H}(z)=\hat{H}_{k_{1}}(z)+\cdots+\hat{H}_{k_{r}}(z)$ such that for $d$ not a singular direction, there exists an unique $r$-tuple of matrices $\left(H_{k_{1}}^{d}(z), \ldots, H_{k_{r}}^{d}(z)\right)$ such that $H_{k_{i}}^{d}(z)$ is analytic on the sector

$$
V_{d}=\Sigma\left(d-\pi / 2 k_{i}, d+\pi / 2 k_{i}\right)
$$

and is $k_{i}$-Gevrey asymptotic to $\hat{H}_{k_{i}}(z)=\sum_{n \in \mathbb{N}} \hat{H}_{n, k_{i}} z^{n}$ on $V_{d}$ : for every closed subsector $W$ of $V_{d}$, there exist $A_{W} \in \mathbb{R}$ and $\varepsilon>0$ such that for all $N$ and all $z \in W$ with $|z|<\varepsilon$,

$$
\left|H_{k_{i}}^{d}(z)-\sum_{n=0}^{N-1} \hat{H}_{n, k_{i}} z^{n}\right| \leq\left(A_{W}\right)^{N} \Gamma\left(1+\frac{N}{k_{i}}\right)|z|^{N},
$$

where $\Gamma$ denotes the gamma function. Until the end of the paper, we will denote a fixed branch of the complex $\log$ arithm by $\log (z)$. Furthermore, the matrix

$$
\left(H_{k_{1}}^{d}(z)+\cdots+H_{k_{r}}^{d}(z)\right) e^{L \log (z)} e^{Q(z)}=H^{d}(z) e^{L \log (z)} e^{Q(z)},
$$

which is analytic on the sector $\Sigma\left(d-\pi / 2 k_{r}, d+\pi / 2 k_{r}\right)$, is a solution of $\partial_{z} Y(z)=$ $A(z) Y(z)$. As a matter of fact, $H_{k_{i}}^{d}(z)$ is $k_{i}$-Gevrey asymptotic to $\hat{H}_{k_{i}}(z)$ on the larger sector

$$
\Sigma\left(d_{l}-\pi / 2 k_{i}, d_{l+1}+\pi / 2 k_{i}\right),
$$

where $d_{l}, d_{l+1}$ are two singular directions such that $] d_{l}, d_{l+1}$ [ contains no singular directions. Therefore, we can construct an analytic solution on the sector $\Sigma\left(d_{l}-\pi / 2 k_{r}, d_{l+1}+\pi / 2 k_{r}\right)$. Let $d \in \mathbb{R}$, and choose $d^{ \pm}$such that

$$
d-\frac{\pi}{2 k_{r}}<d^{-}<d<d^{+}<d+\frac{\pi}{2 k_{r}}
$$

and such that there are no singular directions in $\left[d^{-}, d[\cup] d, d^{+}\right]$. We get two matrices, $H^{d^{+}}(z) e^{L \log (z)} e^{Q(z)}$ and $H^{d^{-}}(z) e^{L \log (z)} e^{Q(z)}$, which are germs of analytic solutions on the sectors

$$
\Sigma\left(d^{-}-\pi / 2 k_{r}, d+\pi / 2 k_{r}\right) \quad \text { and } \quad \Sigma\left(d-\pi / 2 k_{r}, d^{+}+\pi / 2 k_{r}\right),
$$

respectively. The two matrices are, in particular, germs of solutions of $\partial_{z} Y(z)=$ $A(z) Y(z)$ on the sector

$$
\Sigma\left(d-\pi / 2 k_{r}, d+\pi / 2 k_{r}\right) .
$$


A computation shows that there exists a matrix $\mathrm{St}_{d} \in \mathrm{GL}_{m}(\mathbb{C})$, which we call the Stokes matrix in the direction $d$, such that

$$
H^{d^{+}}(z) e^{L \log (z)} e^{Q(z)}=H^{d^{-}}(z) e^{L \log (z)} e^{Q(z)} \mathrm{St}_{d} .
$$

Proposition 1.8. The following statements are equivalent:

(1) The entries of $\hat{H}(z)$ converge.

(2) $\mathrm{St}_{d}=\mathrm{Id}$ for all $d \in \mathbb{R}$.

(3) $\mathrm{St}_{d}=\mathrm{Id}$ for all singular directions.

Proof. From what is preceding, we deduce that if $d$ is not a singular direction, then $\mathrm{St}_{d}=\mathrm{Id}$. Therefore, the statements (2) and (3) are equivalent. If the entries of $\hat{H}(z)$ converge, then, since $\hat{H}(z)$ is Gevrey asymptotic to itself on every sector of $\widetilde{\mathbb{C}}, H^{d}(z)=\hat{H}(z)$ for all $d \in \mathbb{R}$, and (2) holds. Assume now that $\mathrm{St}_{d}=\mathrm{Id}$ for all singular directions. From the proof of [van der Put and Singer 2003, Theorem 8.10], we obtain that the entries of $\hat{H}(z)$ converge.

We can compute the asymptotic solutions using the Laplace and the Borel transformations. See Chapters 2 and 3 of [Balser 1994] for more details.

Definition 1.9. (1) Let $k \in \mathbb{Q}$. The formal Borel transform $\hat{\mathscr{B}}_{k}$ is the map that transforms the formal power series $\sum a_{n} z^{n}$ into the formal power series

$$
\hat{\mathscr{S}}_{k}\left(\sum a_{n} z^{n}\right)=\sum \frac{a_{n}}{\Gamma(1+n / k)} z^{n} .
$$

(2) Let $d \in \mathbb{R}, k \in \mathbb{Q}, \varepsilon>0$ and let $f$ be analytic on the sector $\Sigma(d-\varepsilon, d+\varepsilon)$. We assume that there exist $A, B>0$ such that

$$
|f(z)| \leq A e^{B|z|^{k}}
$$

for $\arg (z)=d$. Then the following integral is the germ of an analytic function on $\Sigma(d-\pi / 2 k, d+\pi / 2 k)$ (see [ibid., p. 13], for a proof), and is called the Laplace transform of order $k$ in the direction $d$ of $f$ :

$$
\mathscr{L}_{k, d}(f)(z)=\int_{0}^{\infty e^{i d}} f(u) e^{-(u / z)^{k}} d\left(\left(\frac{u}{z}\right)^{k}\right) .
$$

For a proof of the following proposition, see Section 7.2 of [ibid.].

Proposition 1.10. Let $k_{1}<\cdots<k_{r}$ be the levels of $\partial_{z} Y(z)=A(z) Y(z)$ and set $k_{r+1}=+\infty$. Suppose that $d \in \mathbb{R}$ is not a singular direction, and let $\hat{h}(z)$ be an entry of $\hat{H}(z)$. Define $\left(\kappa_{1}, \ldots, \kappa_{r}\right)$ by

$$
\kappa_{i}^{-1}=k_{i}^{-1}-k_{i+1}^{-1} .
$$


The series $\hat{\mathscr{B}}_{\kappa_{r}} \circ \cdots \circ \hat{\mathscr{S}}_{\kappa_{1}}(\hat{h})$ converges, and there exist $\varepsilon_{1}, A_{1}, B_{1}>0$ such that it has an analytic continuation $h_{1}$ on the sector $\Sigma\left(d-\varepsilon_{1}, d+\varepsilon_{1}\right)$, and

$$
\left|h_{1}(z)\right| \leq A_{1} e^{B_{1}|z|^{\kappa_{1}}}
$$

in this sector. Moreover, for $j=2, \ldots, r$ there exist $\varepsilon_{j}, A_{j}, B_{j}>0$ such that the function $h_{j+1}=\mathscr{L}_{\kappa_{j}, d}\left(h_{j}\right)$ is analytic on the sector $\Sigma\left(d-\varepsilon_{j}, d+\varepsilon_{j}\right)$, and

$$
\left|h_{j}(z)\right| \leq A_{j} e^{B_{j}|z|^{\kappa_{j}}}
$$

on this sector. Therefore, we may apply $\mathscr{L}_{\kappa_{r}, d} \circ \cdots \circ \mathscr{L}_{\kappa_{1}, d} \circ \hat{\mathscr{P}}_{\kappa_{r}} \circ \cdots \circ \hat{\mathscr{B}}_{\kappa_{1}}$ to every entry of $\hat{H}(z)$. We have the following equality:

$$
H^{d}(z)=\mathscr{L}_{\kappa_{r}, d} \circ \cdots \circ \mathscr{L}_{\kappa_{1}, d} \circ \hat{\mathscr{B}}_{\kappa_{r}} \circ \cdots \circ \hat{\mathscr{B}}_{\kappa_{1}}(\hat{H}) .
$$

1D. Stokes phenomenon in the parametrized case. Consider the equation $(*)$, with $A(z, t) \in \mathrm{M}_{m}\left(\mathscr{O}_{U}(\{z\})\right)$ (see page 90$)$, where $U$ is a nonempty polydisc in $\mathbb{C}^{n}$, and consider $F(z, t)=\hat{H}(z, t) z^{L(t)} e(Q(z, t))$, with $Q(z, t)=\operatorname{Diag}\left(q_{i}(z, t)\right)$, the fundamental solution of Proposition 1.3. Since for all $k \in \mathbb{N}, F(z, t)$ is equal to $\hat{H}(z, t) \operatorname{Diag}\left(z^{k}\right) z^{L(t)-k \operatorname{Id}} e(Q(z, t))$, we may assume that $\hat{H}(z, t)$ has no pole at $z=0$. We define the levels of the system $(*)$ as the levels of the specialized system. The levels may depend upon $t$, but they are invariant on the complement of a closed set with empty interior. We want to extend the definition of the singular directions to the parametrized case. Consider $q(z, t)=q_{k}(t) z^{-k / v}+\cdots+q_{1}(t) z^{-1 / v} \in \mathbf{E}_{U}$. A continuous function $d: U \rightarrow \mathbb{R}$ is called singular for $q(z, t)$ if

$$
q_{k}(t) e^{-i d(t) k / v} \in \mathbb{R}^{\geq 0} \quad \text { for all } t \in U .
$$

In general, the positive number $q_{k}(t) e^{-i d(t) k / v}$ depends on $t$ if $d(t)$ is a singular direction for $q(z, t)$. The singular directions of $(*)$ (we will just write singular directions when no confusion is likely to arise) are the directions that are singular for one of the $q_{i}(z, t)-q_{j}(z, t)$, with $i \neq j$.

Remark 1.11. (1) It may happen that for some $t_{0} \in U$, the singular directions of $(*)$ evaluated at $t_{0}$ are not equal to the singular directions of the specialized system $\partial_{z} Y\left(z, t_{0}\right)=A\left(z, t_{0}\right) Y\left(z, t_{0}\right)$. Take for example $n=1, U=\mathbb{C}, t_{0}=0$ and $A(z, t)=\operatorname{Diag}\left(-2 t z^{-3}-z^{-2}, 2 t z^{-3}+z^{-2}\right)$. The two exponentials are

$$
e\left(q_{1}(z, t)\right)=e\left(t z^{-2}+z^{-1}\right) \text { and } e\left(q_{2}(z, t)\right)=e\left(-t z^{-2}-z^{-1}\right) .
$$

However, there exists $V \subset U$, a closed set with empty interior, such that for all $t_{0}$ in $U \backslash V$, the singular directions of (*) evaluated at $t_{0}$ are equal to the singular directions of the specialized system $\partial_{z} Y\left(z, t_{0}\right)=A\left(z, t_{0}\right) Y\left(z, t_{0}\right)$.

(2) Unfortunately, two different singular directions may be equal on a subset of $U$. For example, for $n=1, U=\mathbb{C}^{*}$, and $A(z, t)=\operatorname{Diag}\left(z^{-2}, t z^{-2},-t z^{-2}\right)$, we find 
three exponentials: $e^{-1 / z}, e^{t / z}$ and $e^{-t / z}$. For $t \in \mathbb{R}^{>0}$, the singular directions of $(2 t) z^{-1}$ are the same as the singular directions of $(t+1) z^{-1}$.

Let $\left(d_{i}(t)\right)_{i \in \mathbb{N}}$ be the singular directions, and

$\mathscr{D}=\left\{t \in U \mid\right.$ there exist $j, j^{\prime} \in \mathbb{N}$ such that $d_{j} \not \equiv d_{j^{\prime}}$ and $\left.d_{j}(t)=d_{j^{\prime}}(t)\right\}$.

Lemma 1.12. $\mathscr{D}$ is a closed subset of $U$ with empty interior.

Proof. Assume that there exist a nonempty polydisc $D \subset \mathscr{D}$ and two singular directions $d_{j}(t), d_{j^{\prime}}(t)$ such that $d_{j}(t)=d_{j^{\prime}}(t)$ on $D$. Then there exist a nonempty polydisc $D^{\prime} \subset D$ and $q(t), q^{\prime}(t) \in M_{D^{\prime}}$ that do not vanish on $D^{\prime}$ such that $q(t) / q^{\prime}(t)$ has constant argument on $D^{\prime}$. An analytic function with constant argument on a polydisc is constant. Hence, we deduce that $d_{j}(t)=d_{j^{\prime}}(t)$ on a polydisc, which implies that $d_{j}(t)=d_{j^{\prime}}(t)$ on $U$. Since the set of singular directions is finite modulo $2 \pi v$ with $v \in \mathbb{N}^{*}$, $\mathscr{D}$ has empty interior.

Thus, if we take a smaller nonempty polydisc $U$, we may assume the following:

- $\mathscr{D}=\varnothing$.

- The levels of $(*)$ are independent of $t$

- For all $t_{0} \in U$, the singular directions of $(*)$ evaluated at $t_{0}$ are equal to the singular directions of the specialized system $\partial_{z} Y\left(z, t_{0}\right)=A\left(z, t_{0}\right) Y\left(z, t_{0}\right)$.

Let $\hat{H}(z, t) z^{L(t)} e(Q(z, t)) \in \mathrm{GL}_{m}\left(\left(\mathbf{K}_{U}\right)^{\wedge}\right)$ be a fundamental solution to the parametrized linear differential system $(*)$ in the same form as in Proposition 1.3, where we consider $A(z, t) \in \mathrm{M}_{m}\left(O_{U}(\{z\})\right)$. Let $d(t)$ be a singular direction, and let $k_{1}<\cdots<k_{r}$ be the levels of $(*)$. For $t$ belonging to $U$, we define the parametrized Stokes matrix $\mathrm{St}_{d(t)}$ (we will just call it the Stokes matrix when no confusion is likely to arise) as $t \mapsto \mathrm{St}_{d(t)}$, where $\mathrm{St}_{d(t)}$ is the Stokes matrix of the specialized system defined just before Proposition 1.8.

Proposition 1.13. Let $d(t)$ be continuous in $t$ such that for all $t_{0}$ in $U, d\left(t_{0}\right)$ is not a singular direction of the unparametrized linear differential equation $\partial_{z} Y\left(z, t_{0}\right)=$ $A\left(z, t_{0}\right) Y\left(z, t_{0}\right)$. We define $t \mapsto H^{d(t)}(z, t) e^{L(t) \log (z)} e^{Q(z, t)}$ as the solution (1-2) of the specialized system. Let $d_{1}(t), d_{2}(t)$ be two singular directions such that for all $t \in U, d_{1}(t)<d(t)<d_{2}(t)$ and $] d_{1}(t), d_{2}(t)$ [ contains no singular directions. Then, there exists a map $U \rightarrow \mathbb{R}^{>0}, t \mapsto \varepsilon(t)$, which is not necessarily continuous, such that $H^{d(t)}(z, t) e^{L(t) \log (z)} e^{Q(z, t)}$ is meromorphic in $(z, t)$ for

$$
z \in \Sigma\left(d_{1}(t)-\pi / 2 k_{r}, d_{2}(t)+\pi / 2 k_{r}\right) \text { with } 0<|z|<\varepsilon(t) \text { and } t \in U .
$$

Notice that the facts that $\mathscr{D}=\varnothing$ and that the singular directions are continuous in $t$ implies the existence of a continuous function $d(t)$ such that, for all $t_{0}$ in $U$, $d\left(t_{0}\right)$ is not a singular direction of the unparametrized linear differential equation $\partial_{z} Y\left(z, t_{0}\right)=A\left(z, t_{0}\right) Y\left(z, t_{0}\right)$. 
Proof. We recall that we have assumed that for all $t_{0} \in U$, the singular directions of $(*)$ evaluated at $t_{0}$ are equal to the singular directions of the specialized system $\partial_{z} Y\left(z, t_{0}\right)=A\left(z, t_{0}\right) Y\left(z, t_{0}\right)$. We have seen in Section 1C that, for $t$ fixed, the asymptotic solution is a germ of meromorphic function on the sector

$$
\Sigma\left(d_{1}(t)-\pi / 2 k_{r}, d_{2}(t)+\pi / 2 k_{r}\right) .
$$

We may replace $d(t)$ by any function, possibly discontinuous, such that for all $t \in U$, $d_{1}(t)<d(t)<d_{2}(t)$. Since the singular directions are continuous in $t$, we may assume that $d(t)$ is locally constant. Since for $z \neq 0, t \mapsto e^{L(t) \log (z)} e^{Q(z, t)} \in M_{U}$, this is now a consequence of Proposition 1.10 and Lemma 1.14 below.

Lemma 1.14. We keep the same notation as in Definition 1.9 and Proposition 1.10. Let $\hat{h}(z, t)$ be one of the entries of $\hat{H}(z, t)$. Let $V \subset U$ be a nonempty polydisc, and let $d \in \mathbb{R}$ such that for all $t \in V, d$ is not an unparametrized singular direction of $(*)$. Then there exists a map $U \rightarrow \mathbb{R}^{>0}, t \mapsto \varepsilon(t)$, which is not necessarily continuous, such that

$$
\mathscr{L}_{\kappa_{r}, d} \circ \cdots \circ \mathscr{L}_{\kappa_{1}, d} \circ \hat{\mathscr{B}}_{\kappa_{r}} \circ \cdots \circ \hat{\mathscr{B}}_{\kappa_{1}}(\hat{h})
$$

is meromorphic in $(z, t)$ for

$$
z \in \Sigma\left(d-\pi / 2 k_{r}, d+\pi / 2 k_{r}\right) \text { with } 0<|z|<\varepsilon(t) \text { and } t \in V .
$$

Moreover, for all $j \leq n$,

$\mathscr{L}_{\kappa_{r}, d} \circ \cdots \circ \mathscr{L}_{\kappa_{1}, d} \circ \hat{\mathscr{P}}_{\kappa_{r}} \circ \cdots \circ \hat{\mathscr{P}}_{\kappa_{1}}\left(\partial_{t_{j}} \hat{h}\right)=\partial_{t_{j}}\left(\mathscr{L}_{\kappa_{r}, d} \circ \cdots \circ \mathscr{L}_{\kappa_{1}, d} \circ \hat{\mathscr{B}}_{\kappa_{r}} \circ \cdots \circ \hat{\mathscr{B}}_{\kappa_{1}}(\hat{h})\right)$.

Proof. We will proceed in two steps.

Step 1: We recall that $\hat{h}(z, t) \in \hat{K}_{U}\left[z^{1 / \nu}\right]$ (where $v \in \mathbb{N}^{*}$ has been defined in Proposition 1.3) and (see Remark 1.7) all the $z$-coefficients are analytic on $U$. Because of Proposition 1.10, for $t$ fixed, $\hat{\mathscr{S}}_{\kappa_{r}} \circ \cdots \circ \hat{\mathscr{P}}_{\kappa_{1}}(\hat{h})$ is a germ of a meromorphic function. Therefore, it belongs to $\mathscr{O}_{U}(\{z\})\left[z^{1 / v}\right]$. Let $h_{1}$ be the analytic continuation defined in Proposition 1.10. In particular, for all $z \in \widetilde{\mathbb{C}}$ with $\arg (z)=d$, $t \mapsto h_{1}(z, t) \in \mathcal{M}_{V}$. The fact that we have a meromorphic function allows us to differentiate termwise, and for all $j \leq n, \partial_{t_{j}} h_{1}$ is equal to the analytic continuation of

$$
\hat{\mathscr{B}}_{\kappa_{r}} \circ \cdots \circ \hat{\mathscr{B}}_{\kappa_{1}}\left(\partial_{t_{j}} \hat{h}\right) \text {. }
$$

Step 2: Let $h_{2}, \ldots, h_{r}$ be the successive Laplace transforms that were defined in Proposition 1.10. Let $t_{0} \in V$, let $W_{t_{0}}$ be a compact neighborhood of $t_{0}$ in $V$, let $i \leq r$, and assume that for $z \in \widetilde{\mathbb{C}}$ with $\arg (z)=d, t \mapsto h_{i}(z, t)$ is meromorphic on $W_{t_{0}}$. It is sufficient to prove that, for all $z \in \widetilde{\mathbb{C}}$ with $\arg (z) \in] d-\pi / 2 \kappa_{i}, d+\pi / 2 \kappa_{i}$ [ and $|z|$ sufficiently small, $t \mapsto h_{i+1}(z, t)$ is meromorphic on $W_{t_{0}}$, and for all $j \leq n$,

$$
\mathscr{L}_{\kappa_{i}, d}\left(\partial_{t_{j}} h_{i}\right)=\partial_{t_{j}}\left(\mathscr{L}_{\kappa_{i}, d}\left(h_{i}\right)\right)=\partial_{t_{j}} h_{i+1} .
$$


The function $\mathscr{L}_{\kappa_{i}, d}\left(h_{i}\right)$ is an integral of a meromorphic function depending analytically upon parameters, and we just have to prove that it is possible to find a function $f$ such that, for all $t \in W_{t_{0}},\left|h_{i}(u, t)\right|<|f(u)|$ and for $\left.\arg (z) \in\right] d-\pi / 2 \kappa_{i}, d+\pi / 2 \kappa_{i}[$, $|z|$ sufficiently small, $\mathscr{L}_{\kappa_{i}, d}(|f|)(z)<\infty$. From Proposition 1.10 , we obtain the existence of $A(t), B(t)>0$ such that for $\arg (u)=d,\left|h_{i}(u, t)\right| \leq A(t) e^{B(t)|u|^{\kappa_{i}}}$. Since $h_{i}(u, t)$ is meromorphic, we may assume that $A(t)$ and $B(t)$ are continuous on $W_{t_{0}}$. The functions $A(t)$ and $B(t)$ admit a maximum $A$ and $B$ on the compact set $W_{t_{0}}$. Finally, for $\left.\arg (z) \in\right] d-\pi / 2 \kappa_{i}, d+\pi / 2 \kappa_{i}$ [ and $|z|$ sufficiently small,

$$
\begin{aligned}
\left|\mathscr{L}_{\kappa_{i}, d} h_{i}\right| & =\left|\int_{0}^{\infty e^{i d}} h_{i}(u, t) e^{-(u / z)^{\kappa_{i}}} d\left(\left(\frac{u}{z}\right)^{\kappa_{i}}\right)\right| \\
& \leq \int_{0}^{\infty} A e^{B|u|^{\kappa_{i}}}\left|e^{-(u / z)^{\kappa_{i}}}\right| d\left(\left(\frac{u}{z}\right)^{\kappa_{i}}\right)<\infty .
\end{aligned}
$$

\section{Parametrized differential Galois theory}

In this section we are interested in parametrized differential Galois theory: this is a generalization of differential Galois theory for parametrized linear differential equations. In Section 2A, we review the parametrized differential Galois theory developed in [CS]. In Section 2B, we prove that some of the results of Section 2A stay valid without the assumption that the field of constants is differentially closed. This will help us in Section $2 \mathrm{C}$ to prove that the local analytic parametrized differential Galois group descends to a smaller field, whose field of constants is not differentially closed. In Section 2D, we explain the main result of the paper: we show an analogue of the density theorem of Ramis in the parametrized case. In Section 2E, we give a similar result for the global parametrized differential Galois group. We end by giving various examples of computation of parametrized differential Galois groups using the parametrized density theorem.

2A. Basic facts. We recall some facts from [CS] about Galois theory of parametrized linear differential equations. Classical Galois theory of unparametrized linear differential equation is presented in some books, such as [van der Put and Singer 2003; Magid 1994].

Let $K$ be a differential field of characteristic 0 with $n+1$ commuting derivations $\partial_{0}, \ldots, \partial_{n}$. We want to study differential equations of the form $\partial_{0} Y=A Y$, with $A \in \mathrm{M}_{m}(K)$. Let $C_{K}$ be the field of constants with respect to $\partial_{0}$. Since all the derivations commute with $\partial_{0},\left(C_{K}, \partial_{1}, \ldots, \partial_{n}\right)$ is a differential field. By abuse of notation, we will sometimes start from a $\left(\partial_{1}, \ldots, \partial_{n}\right)$-differential field $C_{K}$ and build a $\left(\partial_{0}, \ldots, \partial_{n}\right)$-differential field extension $K$ of $C_{K}$, such that $C_{K}$ is the field of constants with respect to $\partial_{0}$.

Example 2.1. If $K=\hat{K}_{U}$, then $\partial_{0}=\partial_{z},\left\{\partial_{1}, \ldots, \partial_{n}\right\}=\Delta_{t}$, and $C_{K}=\mu_{U}$. 
A parametrized Picard-Vessiot extension for the parametrized linear differential equation $\partial_{0} Y=A Y$ on $K$ is a $\left(\partial_{0}, \ldots, \partial_{n}\right)$-differential field extension $\widetilde{K} \mid K$ with the following properties:

- There exists a fundamental solution for $\partial_{0} Y=A Y$ in $\widetilde{K}$, i.e., an invertible matrix $U=\left(u_{i, j}\right)$, with entries in $\widetilde{K}$, such that $\partial_{0} U=A U$.

- $\widetilde{K}=K\left\langle u_{i, j}\right\rangle_{\partial_{0}, \ldots, \partial n}$, i.e., $\widetilde{K}$ is the $\left(\partial_{0}, \ldots, \partial_{n}\right)$-differential field generated by $K$ and the $u_{i, j}$.

- The field of constants of $\widetilde{K}$ with respect to $\partial_{0}$ is $C_{K}$.

Let $L$ be a $\left(\partial_{1}, \ldots, \partial_{n}\right)$-field of characteristic 0 with commuting derivations. The $\left(\partial_{1}, \ldots, \partial_{n}\right)$-differential ring $L\left\{y_{1}, \ldots, y_{k}\right\}_{\partial_{1}, \ldots, \partial_{n}}$ of differential polynomials in $k$ indeterminates over $L$ is the usual polynomial ring in the infinite set of variables

$$
\left\{\partial_{1}^{v_{1}} \ldots \partial_{n}^{v_{n}} y_{j}\right\}_{j \leq k}^{v_{i} \in \mathbb{N}}
$$

and with derivations extending those in $\left\{\partial_{1}, \ldots, \partial_{n}\right\}$ on $L$, defined by

$$
\partial_{i}\left(\partial_{1}^{v_{1}} \ldots \partial_{n}^{v_{n}} y_{j}\right)=\partial_{1}^{v_{1}} \ldots \partial_{i}^{v_{i}+1} \ldots \partial_{n}^{v_{n}} y_{j}
$$

Definition 2.2 [Cassidy and Singer 2007, Definition 3.2]. We say that the field $\left(C_{K}, \partial_{1}, \ldots, \partial_{n}\right)$ is differentially closed if it has the following property: for any $k, l \in \mathbb{N}$ and for all $P_{1}, \ldots, P_{k} \in C_{K}\left\{y_{1}, \ldots, y_{l}\right\}_{\partial_{1}, \ldots, \partial_{n}}$, the system

$$
\left\{\begin{aligned}
P_{1}\left(\alpha_{1}, \ldots, \alpha_{l}\right) & =0 \\
\vdots & \\
P_{k-1}\left(\alpha_{1}, \ldots, \alpha_{l}\right) & =0 \\
P_{k}\left(\alpha_{1}, \ldots, \alpha_{l}\right) & \neq 0,
\end{aligned}\right.
$$

has a solution in $C_{K}$ as soon as it has a solution in a $\left(\partial_{1}, \ldots, \partial_{n}\right)$-differential field containing $C_{K}$.

For simplicity of notation, we will say that $C_{K}$ is differentially closed rather than that $\left(C_{K}, \partial_{1}, \ldots, \partial_{n}\right)$ is differentially closed. Note that there exists a differentially closed extension of $C_{K}$; see [CS, Section 9.1]. By definition, a differentially closed field is algebraically closed.

Proposition 2.3 [CS, Theorem 9.5]. Assume that $C_{K}$ is differentially closed. Then the parametrized Picard-Vessiot extension for $\partial_{0} Y=A Y$ exists and is unique up to $\left(\partial_{0}, \ldots, \partial_{n}\right)$-differential isomorphism.

Until the end of the Section $2 A$, we assume that $C_{K}$ is differentially closed.

Consider $\partial_{0} Y=A Y$ with $A \in \mathrm{M}_{m}(K)$, and let $\widetilde{K} \mid K$ be a parametrized PicardVessiot extension. The parametrized differential Galois group $\operatorname{Gal}_{\partial_{0}}^{\partial_{1}, \ldots, \partial_{n}}(\widetilde{K} \mid K)$ is 
the group of field automorphisms of $\widetilde{K}$ which induce the identity on $K$ and commute with all the derivations. This latter is independent of the choice of the parametrized Picard-Vessiot extension, since all the parametrized Picard-Vessiot extensions are $\left(\partial_{0}, \ldots, \partial_{n}\right)$-differentially isomorphic. In the unparametrized case, the differential Galois group is an algebraic subgroup of $\mathrm{GL}_{m}\left(C_{K}\right)$. In the parametrized case, we find a linear differential algebraic subgroup:

Definition 2.4. Let us consider $m^{2}$ indeterminates $\left(X_{i, j}\right)_{i, j \leq m}$. We say that a subgroup $G$ of $\mathrm{GL}_{m}\left(C_{K}\right)$ is a linear differential algebraic group if there exist $P_{1}, \ldots, P_{k} \in C_{K}\left\{X_{i, j}\right\}_{\partial_{1}, \ldots, \partial_{n}}$ such that for $A=\left(a_{i, j}\right) \in \mathrm{GL}_{m}\left(C_{K}\right)$,

$$
A \in G \Longleftrightarrow P_{1}\left(a_{i, j}\right)=\cdots=P_{k}\left(a_{i, j}\right)=0 .
$$

Let $U$ be a fundamental solution of $\partial_{0} Y=A Y$. One proves directly that the map

$$
\begin{aligned}
\rho_{U}: \mathrm{Gal}_{\partial_{0}}^{\partial_{1}, \ldots, \partial_{n}}(\widetilde{K} \mid K) \longrightarrow \mathrm{GL}_{m}\left(C_{K}\right) \\
\varphi \longmapsto U^{-1} \varphi(U),
\end{aligned}
$$

is an injective group morphism. A fundamental fact is that

$$
\operatorname{Im} \rho_{U}=\left\{U^{-1} \varphi(U) \mid \varphi \in \operatorname{Gal}_{\partial_{0}}^{\partial_{1}, \ldots, \partial_{n}}(\widetilde{K} \mid K)\right\}
$$

is a linear differential algebraic subgroup of $\mathrm{GL}_{m}\left(C_{K}\right)$ (see Theorem 9.5 in [CS]). If we take a different fundamental solution in $\widetilde{K}$, we obtain a conjugate linear differential algebraic subgroup of $\mathrm{GL}_{m}\left(C_{K}\right)$. We will identify $\mathrm{Gal}_{\partial_{0}}^{\partial_{1}, \ldots, \partial_{n}}(\widetilde{K} \mid K)$ with a linear differential algebraic subgroup of $\mathrm{GL}_{m}\left(C_{K}\right)$ for a chosen fundamental solution. We put a topology on $\mathrm{GL}_{m}\left(C_{K}\right)$, called the Kolchin topology, for which the closed sets are defined as the zero loci of finite sets of differential polynomials with coefficients in $C_{K}$.

Example 2.5 [CS, Example 3.1]. Let $n=1$, let $\left(C_{K}, \partial_{t}\right)$ be a differentially closed $\partial_{t^{-}}$field that contains $\left(\mathbb{C}(t), \partial_{t}\right)$, and let us consider $K=C_{K}(z)$, the $\left(\partial_{z}, \partial_{t}\right)$-differential field of rational functions in the indeterminate $z$ with coefficients in $C_{K}$, where $z$ is a $\partial_{t}$-constant with $\partial_{z} z=1, C_{K}$ is the field of constants with respect to $\partial_{z}$, and $\partial_{z}$ commutes with $\partial_{t}$. Let us consider the parametrized differential equation

$$
\partial_{z} Y(z, t)=\frac{t}{z} Y(z, t) .
$$

The fundamental solution is $\left(z^{t}\right)$, and $K\left(z^{t}, \log \right)$ is a parametrized Picard-Vessiot extension (see Section 1A for the notation). Here we have added log because we want the extension to be closed under the derivations $\partial_{z}$ and $\partial_{t}$. Using the fact that the Galois group commutes with $\partial_{z}$ and $\partial_{t}$, we find that the Galois group is given by

$$
\left\{f \in C_{K} \mid f \neq 0 \text { and } f \partial_{t}^{2} f-\left(\partial_{t} f\right)^{2}=0\right\} .
$$


We can see that if we take $C_{K}=\mathbb{C}(t)$ or $C_{K}=M_{\mathbb{C}}$ (see page 90 ), which are not differentially closed, then we find two different groups of differential automorphisms:

$$
\left\{f \in \mathbb{C}(t) \mid f \neq 0 \text { and } f \partial_{t}^{2} f-\left(\partial_{t} f\right)^{2}=0\right\}=\mathbb{C}^{*}
$$

and

$$
\left\{f \in M_{\mathbb{C}} \mid f \neq 0 \text { and } f \partial_{t}^{2} f-\left(\partial_{t} f\right)^{2}=0\right\}=\left\{c e^{b t} \mid b \in \mathbb{C}, c \in \mathbb{C}^{*}\right\},
$$

which shows the importance of considering a Galois group defined over a differentially closed field. See Example 2.26 for the resolution of this ambiguity using the parametrized density theorem.

There is a Galois correspondence theorem for parametrized differential Galois theory; see Theorem 9.5 in [CS]. For a subgroup $G$ of $\mathrm{Gal}_{\partial_{0}}^{\partial_{1}, \ldots, \partial_{n}}(\widetilde{K} \mid K)$, let

$$
\widetilde{K}^{G}=\{a \in \widetilde{K} \mid \sigma(a)=a \text { for all } \sigma \in G\} .
$$

Then the theorem says that the Kolchin-closed subgroups of $\mathrm{Gal}_{\partial_{0}}^{\partial_{1}, \ldots, \partial_{n}}(\widetilde{K} \mid K)$ are in bijection with the $\left(\partial_{0}, \ldots, \partial_{n}\right)$-differential subfields of $\widetilde{K}$ containing $K$ via the map

$$
G \mapsto \widetilde{K}^{G}
$$

The inverse map is given by

$$
M \mapsto \mathrm{Gal}_{\partial_{0}}^{\partial_{1}, \ldots, \partial_{n}}(\widetilde{K} \mid M),
$$

where $\mathrm{Gal}_{\partial_{0}}^{\partial_{1}, \ldots, \partial_{n}}(\widetilde{K} \mid M)$ denotes the set of elements of $\mathrm{Gal}_{\partial_{0}}^{\partial_{1}, \ldots, \partial_{n}}(\widetilde{K} \mid K)$ inducing the identity map on $M$. In particular, we have the following corollary:

Corollary 2.6. Let $G$ be an arbitrary subgroup of $\mathrm{Gal}_{\partial_{0}}^{\partial_{1}, \ldots, \partial_{n}}(\widetilde{K} \mid K)$. Then $\widetilde{K}^{G}=K$ if and only if $G$ is dense for the Kolchin topology in $\mathrm{Gal}_{\partial_{0}}^{\partial_{1}, \ldots, \partial_{n}}(\widetilde{K} \mid K)$.

Let $L|M| K$ be $\left(\partial_{1}, \ldots, \partial_{n}\right)$-differential field extensions. Notice that we do not exclude $L=M=K$. All the definitions that we give before the next proposition come from [Hardouin and Singer 2008, §6.2.3].

We remark that $P\left(a_{1}, \ldots, a_{n}\right)$ is well-defined for $P \in M\left\{X_{1}, \ldots, X_{k}\right\}_{\partial_{1}, \ldots, \partial_{n}}$ and $a_{1}, \ldots, a_{k} \in L$. Then we may define the $\left(\partial_{1}, \ldots, \partial_{n}\right)$-differential transcendence degree of $L$ over $M$ as the maximum number of elements $a_{1}, \ldots, a_{k}$ of $L$ such that

$$
P\left(a_{1}, \ldots, a_{k}\right) \neq 0,
$$

for all nonzero $\left(\partial_{1}, \ldots, \partial_{n}\right)$-differential polynomials $P$ with coefficients in $M$. The $\left(\partial_{1}, \ldots, \partial_{n}\right)$-differential transcendence degree of an integral domain over another integral domain is defined to be the $\left(\partial_{1}, \ldots, \partial_{n}\right)$-differential transcendence degree of the fraction field of the first one over the fraction field of the second one. 
Let us consider $m^{2}$ indeterminates $\left(X_{i, j}\right)_{i, j \leq m}$. Let $(p)$ be a prime $\left(\partial_{1}, \ldots, \partial_{n}\right)$ differential ideal of $C_{K}\left\{X_{i, j}\right\}_{\partial_{1}, \ldots, \partial_{n}}$, i.e., a prime ideal stable under the derivations $\partial_{1}, \ldots, \partial_{n}$. The $\left(\partial_{1}, \ldots, \partial_{n}\right)$-dimension of $(p)$ over $C_{K}$ is defined to be the $\left(\partial_{1}, \ldots, \partial_{n}\right)$-differential transcendence degree of the quotient ring

$$
C_{K}\left\{X_{i, j}\right\}_{\partial_{1}, \ldots, \partial_{n}} /(p)
$$

over $C_{K}$.

Let $(r)$ be a radical $\left(\partial_{1}, \ldots, \partial_{n}\right)$-differential ideal of $C_{K}\left\{X_{i, j}\right\}_{\partial_{1}, \ldots, \partial_{n}}$, i.e., a radical ideal stable under the derivations $\partial_{1}, \ldots, \partial_{n}$. Let $\left(p_{1}\right), \ldots,\left(p_{v}\right)$ with $v \in$ $\mathbb{N}^{*}$ be the prime $\left(\partial_{1}, \ldots, \partial_{n}\right)$-differential ideals such that $(r)=\bigcap_{k \leq v}\left(p_{k}\right)$. The $\left(\partial_{1}, \ldots, \partial_{n}\right)$-dimension of $(r)$ over $C_{K}$ is defined to be the maximum in $k$ of the $\left(\partial_{1}, \ldots, \partial_{n}\right)$-dimension of $\left(p_{k}\right)$ over $C_{K}$.

Assume that $M \subset \widetilde{K}$. Let $(q)$ be the radical $\left(\partial_{1}, \ldots, \partial_{n}\right)$-differential ideal of $C_{K}\left\{X_{i, j}\right\}_{\partial_{1}, \ldots, \partial_{n}}$ that defines $\mathrm{Gal}_{\partial_{0}}^{\partial_{1}, \ldots, \partial_{n}}(\widetilde{K} \mid M)$ (see the proof of Proposition 9.10 in $[\mathrm{CS}])$. We define the $\left(\partial_{1}, \ldots, \partial_{n}\right)$-differential dimension of $\mathrm{Gal}_{\partial_{0}}^{\partial_{1}, \ldots, \partial_{n}}(\widetilde{K} \mid M)$ over $C_{K}$ as the $\left(\partial_{1}, \ldots, \partial_{n}\right)$-dimension of $(q)$ over $C_{K}$.

Proposition 2.7 [Hardouin and Singer 2008, Proposition 6.26]. The $\left(\partial_{1}, \ldots, \partial_{n}\right)$ differential transcendence degree of $\widetilde{K}$ over $M$ is equal to the $\left(\partial_{1}, \ldots, \partial_{n}\right)$-differential dimension of $\mathrm{Gal}_{\partial_{0}}^{\partial_{1}, \ldots, \partial_{n}}(\widetilde{K} \mid M)$ over $C_{K}$.

Example 2.5 revisited. Let us keep the same notation as in Example 2.5. The parametrized Picard-Vessiot extension is $K\left(z^{t}, \log \right)$ and the Galois group is

$$
\left\{f \in C_{K} \mid f \neq 0 \text { and } f \partial_{t}^{2} f-\left(\partial_{t} f\right)^{2}=0\right\} .
$$

We may directly check that the $\partial_{t}$-differential dimension of the Galois group is 0 , and therefore $z^{t}$ satisfies a $\partial_{t}$-differential polynomial equation with coefficients in $C_{K}$.

\section{B. Parametrized differential Galois theory for a nondifferentially closed field}

of constants. Let $K$ be a differential field of characteristic 0 with $n+1$ commuting derivations $\partial_{0}, \ldots, \partial_{n}$. Let $C_{K}$ be the field of constants with respect to $\partial_{0}$. Note that we do not assume $C_{K}$ to be differentially closed. Consider $\partial_{0} Y=A Y$, with $A \in \mathrm{M}_{m}(K)$, and assume the existence of $\widetilde{K} \mid K$, a parametrized Picard-Vessiot extension for $\partial_{0} Y=A Y$ (see Section 2A). This means in particular that the field of constants of $\widetilde{K}$ with respect to $\partial_{0}$ is $C_{K}$. Let $F=\left(F_{i, j}\right) \in \operatorname{GL}_{m}(\widetilde{K})$ be a fundamental solution such that $\widetilde{K}=K\left\langle F_{i, j}\right\rangle_{\partial_{0}, \ldots, \partial n}$ (see Section 2 A for the notation). Let $\operatorname{Aut}_{\partial_{0}}^{\partial_{1}, \ldots, \partial_{n}}(\widetilde{K} \mid K)$ be the group of $\left(\partial_{0}, \ldots, \partial_{n}\right)$-differential field automorphisms of $\widetilde{K}$ keeping $K$ invariant.

Remark 2.8. We avoid here the notation $\mathrm{Gal}_{\partial_{0}}^{\partial_{1}, \ldots, \partial_{n}}(\widetilde{K} \mid K)$, because we have no theorem that guarantees the uniqueness of the parametrized Picard-Vessiot extension 
$\widetilde{K} \mid K$, since $C_{K}$ is not differentially closed. However, we will call it the parametrized differential Galois group, or Galois group, if no confusion is likely to arise.

We extend Definition 2.4 for the field $C_{K}$. Let us consider $m^{2}$ indeterminates $\left(X_{i, j}\right)_{i, j \leq m}$. We say that a subgroup $G$ of $\mathrm{GL}_{m}\left(C_{K}\right)$ is a linear differential algebraic group if there exist $P_{1}, \ldots, P_{k} \in C_{K}\left\{X_{i, j}\right\}_{\partial_{1}, \ldots, \partial_{n}}$ such that for $A=\left(a_{i, j}\right) \in \mathrm{GL}_{m}\left(C_{K}\right)$,

$$
A \in G \Longleftrightarrow P_{1}\left(a_{i, j}\right)=\cdots=P_{k}\left(a_{i, j}\right)=0 .
$$

The goal of the subsection is to prove:

Proposition 2.9. (1) Let us consider the injective group morphism

Then

$$
\begin{gathered}
\rho_{F}: \operatorname{Aut}_{\partial_{0}}^{\partial_{1}, \ldots, \partial_{n}}(\tilde{K} \mid K) \longrightarrow \mathrm{GL}_{m}\left(C_{K}\right) \\
\varphi \longmapsto F^{-1} \varphi(F) .
\end{gathered}
$$

$$
\operatorname{Im} \rho_{F}=\left\{F^{-1} \varphi(F) \mid \varphi \in \operatorname{Aut}_{\partial_{0}}^{\partial_{1}, \ldots, \partial_{n}}(\widetilde{K} \mid K)\right\}
$$

is a linear differential algebraic subgroup of $\mathrm{GL}_{m}\left(C_{K}\right)$. We will identify $\operatorname{Aut}_{\partial_{0}}^{\partial_{1}, \ldots, \partial_{n}}(\widetilde{K} \mid K)$ with a linear differential algebraic subgroup of $\mathrm{GL}_{m}\left(C_{K}\right)$ for a chosen fundamental solution. The image is independent of this choice, up to conjugacy by an element of $\mathrm{GL}_{m}\left(C_{K}\right)$.

(2) Let $G$ be a subgroup of $\operatorname{Aut}_{\partial_{0}}^{\partial_{1}, \ldots, \partial_{n}}(\widetilde{K} \mid K)$. If $\widetilde{K}^{G}=K$, then $G$ is dense in $\operatorname{Aut}_{\partial_{0}}^{\partial_{1}, \ldots, \partial_{n}}(\widetilde{K} \mid K)$ for the Kolchin topology.

Remark that, contrary to Corollary 2.6, the converse of (2) is false when $C_{K}$ is not differentially closed. See [CS, Example 3.1]. Before showing the proposition, we point out two facts we will use in the proof. Let $L \mid K$ be a $\left(\partial_{0}, \ldots, \partial_{n}\right)$-differential field extension and $a_{1}, \ldots, a_{k} \in L$.

- As in the case where $C_{K}$ is differentially closed (see Section $\left.2 \mathrm{~A}\right), P\left(a_{1}, \ldots, a_{k}\right)$ is well-defined for $P \in K\left\{X_{1}, \ldots, X_{k}\right\}_{\partial_{1}, \ldots, \partial_{n}}$.

- The set $\left\{P\left(a_{1}, \ldots, a_{k}\right) \mid P \in K\left\{X_{1}, \ldots, X_{k}\right\}_{\partial_{1}, \ldots, \partial_{n}}\right\}$ is a $\left(\partial_{0}, \ldots, \partial_{n}\right)$-differential field extension we will denote by $L\left\{a_{1}, \ldots, a_{k}\right\}_{\partial_{1}, \ldots, \partial_{n}} \mid L$.

Proof of Proposition 2.9.

Part (1): We follow here the proof of Proposition 9.10 in [CS]. We consider the differential polynomial ring

$$
R=K\left\{X_{i, j}, 1 / \operatorname{det}\left(X_{i, j}\right)\right\}_{\partial_{1}, \ldots, \partial_{n}},
$$

and endow it with the $\partial_{0}$-differential structure defined by $\partial_{0}\left(X_{i, j}\right)=A\left(X_{i, j}\right)$. Let us consider

$$
S=K\left\{F_{i, j}, 1 / \operatorname{det}\left(F_{i, j}\right)\right\}_{\partial_{0}, \ldots, \partial_{n}},
$$


the $\left(\partial_{0}, \ldots, \partial_{n}\right)$-differential subring of $\widetilde{K}$ generated by the $F_{i, j}$ and $1 / \operatorname{det}\left(F_{i, j}\right)$ over $K$. It is an integral domain. Let $q$ be the obvious prime $\left(\partial_{0}, \ldots, \partial_{n}\right)$-differential ideal such that $R / q \simeq S$. Let $Z_{i, j}$ be the image of $X_{i, j}$ in $S \subset \widetilde{K}$, so that $\left(Z_{i, j}\right)$ is a fundamental solution for $\partial_{0} Y=A Y$ in $S$. Consider the following rings:

$$
\begin{array}{cc}
\tilde{K}\left\{X_{i, j}, 1 / \operatorname{det}\left(X_{i, j}\right)\right\}_{\partial_{1}, \ldots, \partial_{n}}= & \widetilde{K}\left\{Y_{i, j}, 1 / \operatorname{det}\left(Y_{i, j}\right)\right\}_{\partial_{1}, \ldots, \partial_{n}} \\
\cup & \cup \\
K\left\{X_{i, j}, 1 / \operatorname{det}\left(X_{i, j}\right)\right\}_{\partial_{1}, \ldots, \partial_{n}} & C_{K}\left\{Y_{i, j}, 1 / \operatorname{det}\left(Y_{i, j}\right)\right\}_{\partial_{1}, \ldots, \partial_{n}},
\end{array}
$$

where the indeterminates $Y_{i, j}$ are defined by $\left(X_{i, j}\right)=\left(Z_{i, j}\right)\left(Y_{i, j}\right)$. We remark that $\partial_{0}\left(Y_{i, j}\right)=0$. Since we consider fields that are of characteristic 0 , the differential ideal

$$
\begin{aligned}
q \widetilde{K}\left\{Y_{i, j}, 1 / \operatorname{det}\left(Y_{i, j}\right)\right\}_{\partial_{1}, \ldots, \partial_{n}} & \subset \widetilde{K}\left\{X_{i, j}, 1 / \operatorname{det}\left(X_{i, j}\right)\right\}_{\partial_{1}, \ldots, \partial_{n}} \\
& =\widetilde{K}\left\{Y_{i, j}, 1 / \operatorname{det}\left(Y_{i, j}\right)\right\}_{\partial_{1}, \ldots, \partial_{n}}
\end{aligned}
$$

is a radical $\left(\partial_{0}, \ldots, \partial_{n}\right)$-differential ideal (see Corollary A.17 in [van der Put and Singer 2003]). The next lemma is an adaptation of Lemma 9.8 in [CS] without the assumption that the field of constants is differentially closed.

Lemma 2.10. The $\left(\partial_{0}, \ldots, \partial_{n}\right)$-ideal $q \widetilde{K}\left\{Y_{i, j}, 1 / \operatorname{det}\left(Y_{i, j}\right)\right\}_{\partial_{1}, \ldots, \partial_{n}}$ is generated by

$$
I=q \widetilde{K}\left\{Y_{i, j}, 1 / \operatorname{det}\left(Y_{i, j}\right)\right\}_{\partial_{1}, \ldots, \partial_{n}} \cap C_{K}\left\{Y_{i, j}, 1 / \operatorname{det}\left(Y_{i, j}\right)\right\}_{\partial_{1}, \ldots, \partial_{n}} .
$$

Proof. Let $\left(e_{i}\right)_{i \in B}$ be a basis of $C_{K}\left\{Y_{i, j}, 1 / \operatorname{det}\left(Y_{i, j}\right)\right\}_{\partial_{1}, \ldots, \partial_{n}}$ over $C_{K}$. Let

$$
f=\sum_{i=1}^{n} m_{i} e_{i} \in q \widetilde{K}\left\{Y_{i, j}, 1 / \operatorname{det}\left(Y_{i, j}\right)\right\}_{\partial_{1}, \ldots, \partial_{n}},
$$

with $m_{i} \in \widetilde{K}$. By induction on $n$ we will show that $f \in I$. If $n=0$ or 1 there is nothing to prove. We assume that $n>1$. We can suppose that $m_{1}=1$ and $m_{2} \notin C_{K}$. Then, since the field of constants of $\widetilde{K}$ with respect to $\partial_{z}$ is $C_{K}$,

$$
\partial_{0}(f)=\sum_{i=2}^{n} \partial_{0}\left(m_{i}\right) e_{i} \neq 0 \quad \text { and } \quad f \in q \widetilde{K}\left\{Y_{i, j}, 1 / \operatorname{det}\left(Y_{i, j}\right)\right\}_{\partial_{1}, \ldots, \partial_{n}} .
$$

Then, by induction, $\partial_{0}(f) \in I$. By the same argument,

$$
\partial_{0}\left(m_{2}^{-1} f\right) \in I \text {. }
$$

Then $\partial_{0}\left(m_{2}^{-1}\right) f=\partial_{0}\left(m_{2}^{-1} f\right)-m_{2}^{-1} \partial_{0} f \in I$. Since $\partial_{0}\left(m_{2}^{-1}\right) \neq 0$, we obtain that $f \in I$.

By Lemma $2.10, q \widetilde{K}\left\{X_{i, j}, 1 / \operatorname{det}\left(X_{i, j}\right)\right\}_{\partial_{1}, \ldots, \partial_{n}}$ is generated by

$$
I=q \widetilde{K}\left\{X_{i, j}, 1 / \operatorname{det}\left(X_{i, j}\right)\right\}_{\partial_{1}, \ldots, \partial_{n}} \cap C_{K}\left\{Y_{i, j}, 1 / \operatorname{det}\left(Y_{i, j}\right)\right\}_{\partial_{1}, \ldots, \partial_{n}} .
$$


Clearly, $I$ is a $\left(\partial_{1}, \ldots, \partial_{n}\right)$-radical ideal of $C_{K}\left\{Y_{i, j}, 1 / \operatorname{det}\left(Y_{i, j}\right)\right\}_{\partial_{1}, \ldots, \partial_{n}}$. Let $C=$ $\left(C_{i, j}\right) \in \mathrm{GL}_{m}\left(C_{K}\right)$. The following statements are equivalent:

(1) $\left(C_{i, j}\right) \in \operatorname{Aut}_{\partial_{0}}^{\partial_{1}, \ldots, \partial_{n}}(\widetilde{K} \mid K)$.

(2) The map $K\left\{X_{i, j}, 1 / \operatorname{det}\left(X_{i, j}\right)\right\}_{\partial_{1}, \ldots, \partial_{n}} \rightarrow K\left\{X_{i, j}, 1 / \operatorname{det}\left(X_{i, j}\right)\right\}_{\partial_{1}, \ldots, \partial_{n}}$ defined by $\left(X_{i, j}\right) \mapsto\left(X_{i, j}\right)\left(C_{i, j}\right):=\left(\sum_{k=1}^{m} X_{i, k} C_{k, j}\right)$ leaves $q$ invariant.

(3) The map $K\left\{X_{i, j}, 1 / \operatorname{det}\left(X_{i, j}\right)\right\}_{\partial_{1}, \ldots, \partial_{n}} \rightarrow \widetilde{K}$ defined by $\left(X_{i, j}\right) \mapsto\left(Z_{i, j}\right)\left(C_{i, j}\right)$ sends $q$ to 0 .

(4) The map $\widetilde{K}\left\{X_{i, j}, 1 / \operatorname{det}\left(X_{i, j}\right)\right\}_{\partial_{1}, \ldots, \partial_{n}} \rightarrow \widetilde{K}$ defined by $\left(X_{i, j}\right) \mapsto\left(Z_{i, j}\right)\left(C_{i, j}\right)$ sends $q \widetilde{K}\left\{X_{i, j}, 1 / \operatorname{det}\left(X_{i, j}\right)\right\}_{\partial_{1}, \ldots, \partial_{n}}=q \widetilde{K}\left\{Y_{i, j}, 1 / \operatorname{det}\left(Y_{i, j}\right)\right\}_{\partial_{1}, \ldots, \partial_{n}}$ to 0.

(5) The map $\widetilde{K}\left\{Y_{i, j}, 1 / \operatorname{det}\left(Y_{i, j}\right)\right\}_{\partial_{1}, \ldots, \partial_{n}} \rightarrow \widetilde{K}$ defined by $\left(Y_{i, j}\right) \mapsto\left(C_{i, j}\right)$ sends $q \widetilde{K}\left\{Y_{i, j}, 1 / \operatorname{det}\left(Y_{i, j}\right)\right\}_{\partial_{1}, \ldots, \partial_{n}}$ to 0 .

The theorem is now a consequence of the fact that $q \widetilde{K}\left\{Y_{i, j}, 1 / \operatorname{det}\left(Y_{i, j}\right)\right\}_{\partial_{1}, \ldots, \partial_{n}}$ is generated by $I$, a $\left(\partial_{1}, \ldots, \partial_{n}\right)$-radical ideal of $C_{K}\left\{Y_{i, j}, 1 / \operatorname{det}\left(Y_{i, j}\right)\right\}_{\partial_{1}, \ldots, \partial_{n}}$.

Part (2): We follow the proof of Proposition 9.10 in [CS], and use the same notation as before. By construction, the ideal $I$ of Lemma 2.10 above is the differential ideal that defines the Galois group. Assume that the Kolchin closure of $G$ is not the whole Galois group. Then there exists $P \in C_{K}\left\{Y_{i, j}, 1 / \operatorname{det}\left(Y_{i, j}\right)\right\}_{\partial_{1}, \ldots, \partial_{n}}$ such that $P \notin I$ and $P(g)=0$ for all $g \in G$. Lemma 2.10 implies that

$$
P \notin J=q \widetilde{K}\left\{Y_{i, j}, 1 / \operatorname{det}\left(Y_{i, j}\right)\right\}_{\partial_{1}, \ldots, \partial_{n}} .
$$

Let $T$ consist of all $Q \in \widetilde{K}\left\{X_{i, j}, 1 / \operatorname{det}\left(X_{i, j}\right)\right\}_{\partial_{1}, \ldots, \partial_{n}}$ such that $Q \notin J$ and

$$
Q\left(\left(Z_{i, j}\right)\left(g_{i, j}\right)\right)=0 \text { for all } g=\left(g_{i, j}\right) \in G .
$$

Since $P \in T, T \neq\{0\}$. An element $Q \in T$ can be written as

$$
Q=f_{1} Q_{1}+\cdots+f_{\nu} Q_{\nu}
$$

where $f_{i} \in \widetilde{K}$ and $Q_{i} \in K\left\{X_{i, j}, 1 / \operatorname{det}\left(X_{i, j}\right)\right\}_{\partial_{1}, \ldots, \partial_{n}}$. Let $Q=f_{1} Q_{1}+\cdots+f_{\nu} Q_{\nu} \in T$ such that:

- $f_{1}=1$,

- all the $f_{i}$ are nonzero,

- $v$ is minimal.

For all $g \in G$, let $Q^{g}=f_{1}^{g} Q_{1}+\cdots+f_{\nu}^{g} Q_{\nu} \in T$. Let $g \in G$. Since $Q-Q^{g}$ is shorter than $Q$, and satisfies $\left(\underset{\widetilde{K}}{Q}-Q^{g}\right)\left(\left(Z_{i, j}\right)\left(g_{i, j}\right)\right)=0$, we have $Q-Q^{g} \in J$. If $Q-Q^{g} \neq 0$, there exists $l \in \widetilde{K}$ such that $Q-l\left(Q-Q^{g}\right)$ is shorter than $Q$. Since $Q-l\left(Q-Q^{g}\right) \in T$, this is not possible unless $Q-Q^{g}=0$. Therefore, $Q=Q^{g}$, for all $g \in G$, and so $Q \in K\left\{X_{i, j}, 1 / \operatorname{det}\left(X_{i, j}\right)\right\}_{\partial_{1}, \ldots, \partial_{n}}$. Since $Q\left(Z_{i, j}\right)=0$, we have $Q \in J$. This completes the proof of Proposition 2.9. 


\section{C. A result of descent for the local analytic parametrized differential Galois}

group. We keep the notations of Section 1. Consider the equation $(*)$ with $A(z, t) \in$ $\mathrm{M}_{m}\left(\mathscr{O}_{U}(\{z\})\right)$, where $U$ is a nonempty polydisc in $\mathbb{C}^{n}$, and $\mathscr{O}_{U}(\{z\})$ has been defined on page 90.

Remark 2.11. Note that $\mathcal{O}_{U}(\{z\})$ is a ring but not a field in general. For example, if $n=1,(z-t)^{-1} \notin \mathscr{O}_{U}(\{z\})$. However, we have $(z-t)^{-1} \in \mathscr{O}_{\mathbb{C}^{*}}(\{z\})$. More generally let $\alpha(z, t) \in \mathrm{O}_{U}(\{z\})$. For $t \in U$, let be $R(t)$ minimal such that $|\alpha(z, t)| \neq 0$ for $0<|z|<R(t)$. There exist a nonempty polydisc $U^{\prime}$ and $\varepsilon>0$ with $R(t)>\varepsilon$ on $U^{\prime}$. In particular, we have $\alpha(z, t)^{-1} \in \mathcal{O}_{U^{\prime}}(\{z\})$.

Since $\mathscr{O}_{U}(\{z\}) \subset \hat{K}_{U}$, which is a field, $\mathscr{O}_{U}(\{z\})$ is an integral domain, and we can define $K_{U}$ as the fraction field of $\mathscr{O}_{U}(\{z\})$. We have

$$
\left\{a \in K_{U} \mid \partial_{z} a=0\right\}=\left\{a \in \hat{K}_{U} \mid \partial_{z} a=0\right\}=M_{U} .
$$

Let

$$
F(z, t)=\left(F_{i, j}\right)=\hat{H}(z, t) z^{L(t)} e(Q(z, t)) \in \mathrm{GL}_{m}\left(\left(\mathbf{K}_{U}\right)^{\wedge}\right) \quad(\text { see Section 1A) }
$$

be the fundamental solution given in Proposition 1.3. Let us denote

$$
K_{U}\left\langle F_{i, j}\right\rangle_{\partial_{z}, \Delta_{t}}=\left(K_{U}\right)^{\sim},
$$

which is a $\left(\partial_{z}, \Delta_{t}\right)$-differential subfield of $\left(\mathbf{K}_{U}\right)^{\wedge}$. We have seen in Section 1A that $\left(\mathbf{K}_{U}\right)^{\wedge}$ has field of constants with respect to $\partial_{z}$ equal to $\mathcal{M}_{U}$. Then we deduce that $\left(K_{U}\right)^{\sim} \mid K_{U}$ is a parametrized Picard-Vessiot extension. Therefore, the results of Section 2B may be applied here; and we can define a parametrized differential Galois group $\operatorname{Aut}_{\partial_{z}}^{\Delta_{t}}\left(\left(K_{U}\right)^{\sim} \mid K_{U}\right)$, which will be identified with a linear differential algebraic subgroup of $\operatorname{GL}_{m}\left(M_{U}\right)$. We want to prove now that it is the "same" as the one of Section 2A.

Let $C$ be a $\left(\Delta_{t}\right)$-differentially closed field that contains $\mu_{U}$. Let us define $C \llbracket z \rrbracket\left[z^{-1}\right]$, the $\left(\partial_{z}, \Delta_{t}\right)$-differential field, where $z$ is a $\left(\Delta_{t}\right)$-constant with $\partial_{z} z=1$, $C$ is the field of constants with respect to $\partial_{z}$, and $\partial_{z}$ commutes with all the derivations. We define the ring $K_{U} \otimes_{\mathcal{M}_{U}} C$ with the differential structure given by

$$
\partial\left(a \otimes_{\mu_{U}} c\right)=\partial a \otimes_{\mathcal{M}_{U}} c+a \otimes_{\mathcal{M}_{U}} \partial c \text { for all } a \in K_{U}, c \in C, \partial \in\left\{\partial_{z}, \Delta_{t}\right\} .
$$

This $\left(\partial_{z}, \Delta_{t}\right)$-differential ring can be naturally embedded into $C \llbracket z \rrbracket\left[z^{-1}\right]$, which implies that it is an integral domain. Therefore we may define $\mathscr{K}_{C, U}$, the field of fractions of $K_{U} \otimes_{\mathcal{M}_{U}} C$. We see now $\mathscr{K}_{C, U}$ (resp. $K_{U} \otimes_{\mathcal{M}_{U}} C$ ) as a subfield (resp. subring) of $C \llbracket z \rrbracket\left[z^{-1}\right]$.

Proposition 2.12. Let us keep the same notation. Consider the equation $\partial_{z} Y(z, t)=$ $A(z, t) Y(z, t)$ with $A(z, t) \in \mathrm{M}_{m}\left(\mathscr{O}_{U}(\{z\})\right)$. The extension field

$$
\mathscr{K}_{C, U}\left\langle F_{i, j}\right\rangle_{\partial_{z}, \Delta_{t}}\left|\mathscr{K}_{C, U}=\left(\mathscr{K}_{C, U}\right)^{\sim}\right| \mathscr{K}_{C, U}
$$


is a parametrized Picard-Vessiot extension for $\partial_{z} Y(z, t)=A(z, t) Y(z, t)$. Moreover, there exist $P_{1}, \ldots, P_{k} \in \mathcal{M}_{U}\left\{X_{i, j}\right\}_{\Delta_{t}}$ such that the image of the representation of $\operatorname{Gal}_{\partial_{z}}^{\Delta_{t}}\left(\left(\mathscr{K}_{C, U}\right)^{\sim} \mid \mathscr{K}_{C, U}\right)$ (resp. Aut $\left.\partial_{\partial_{z}}^{\Delta_{t}}\left(\left(K_{U}\right)^{\sim} \mid K_{U}\right)\right)$ associated to $F(z, t)$ is the set of $C$-rational points (resp. $M_{U}$-rational points) of the linear differential algebraic subgroup of $\mathrm{GL}_{m}(C)$ (resp. $\left.\mathrm{GL}_{m}\left(M_{U}\right)\right)$ defined by $P_{1}, \ldots, P_{k}$. More explicitly,

$$
\begin{aligned}
& \left\{F^{-1} \varphi(F) \mid \varphi \in \mathrm{Gal}_{\partial_{z}}^{\Delta_{t}}\left(\left(\mathcal{K}_{C, U}\right)^{\sim} \mid \mathscr{K}_{C, U}\right)\right\} \\
& \quad=\left\{A=\left(a_{i, j}\right) \in \mathrm{GL}_{m}(C) \mid P_{1}\left(a_{i, j}\right)=\cdots=P_{k}\left(a_{i, j}\right)=0\right\}
\end{aligned}
$$

and

$$
\begin{aligned}
\left\{F^{-1} \varphi(F) \mid \varphi \in \operatorname{Aut}_{\partial_{z}}^{\Delta_{t}}\right. & \left.\left(\left(K_{U}\right)^{\sim} \mid K_{U}\right)\right\} \\
& =\left\{A=\left(a_{i, j}\right) \in \mathrm{GL}_{m}\left(M_{U}\right) \mid P_{1}\left(a_{i, j}\right)=\cdots=P_{k}\left(a_{i, j}\right)=0\right\} .
\end{aligned}
$$

Proof. We follow the proof of [Mitschi and Singer 2012, Proposition 3.3]. Let $\left(d_{k}\right)$ be an $\mathcal{M}_{U}$-basis of $C$. Let us prove that the $d_{k}$ are linearly independent over $\left(K_{U}\right)^{\sim}$. Write $\sum_{k \leq \kappa} d_{k} P_{k}=0$ with $0 \neq P_{k} \in\left(K_{U}\right)^{\sim}, \kappa \geq 2$ minimal and $P_{\kappa}=1$. We have $\sum_{k \leq \kappa-1} d_{k} \partial_{z} P_{k}=0$. If $\kappa=2$, then $\partial_{z} P_{1}=0$. If $\kappa>2$, the minimality of $\kappa$ implies that $\partial_{z} P_{k}=0$ for all $k$. Since $\left(K_{U}\right)^{\sim} \mid K_{U}$ is a parametrized Picard-Vessiot extension, $P_{k} \in \mathcal{M}_{U}$ for all $k$, and the $d_{k}$ are linearly independent over $\left(K_{U}\right)^{\sim}$.

Now, we prove that $\mathscr{K}_{C, U}\left\langle F_{i, j}\right\rangle_{\partial_{z}, \Delta_{t}} \mid \mathscr{K}_{C, U}$ is a parametrized Picard-Vessiot extension for $\partial_{z} Y(z, t)=A(z, t) Y(z, t)$. Let $\alpha \in \mathscr{K}_{C, U}\left\langle F_{i, j}\right\rangle_{\partial_{z}, \Delta_{t}}$ with $\partial_{z} \alpha=0$. We may assume that $\alpha=\sum d_{k} P_{k}$, where $P_{k} \in\left(K_{U}\right)^{\sim}$. We have $\partial_{z} \alpha=\sum d_{k} \partial_{z} P_{k}=0$. Since the $d_{k}$ are linearly independent over $\left(K_{U}\right)^{\sim}$, we find $\partial_{z} P_{k}=0$. Hence, $P_{k} \in \mathcal{M}_{U}$, because $\left(K_{U}\right)^{\sim} \mid K_{U}$ is a parametrized Picard-Vessiot extension. Therefore, $\alpha \in C$ and $\mathscr{K}_{C, U}\left\langle F_{i, j}\right\rangle_{\partial_{z}, \Delta_{t}} \mid \mathscr{K}_{C, U}$ is a parametrized Picard-Vessiot extension for $\partial_{z} Y(z, t)=A(z, t) Y(z, t)$.

Let $Y_{i, j}$ be a set of $m^{2}$ indeterminates and let $I_{0}, I_{1}$ be $\left(\partial_{z}, \Delta_{t}\right)$-differential ideals such that

$$
\begin{gathered}
R_{0}=K_{U}\left\{F_{i, j}\right\}_{\partial_{z}, \Delta_{t}}=K_{U}\left\{Y_{i, j}\right\}_{\partial_{z}, \Delta_{t}} / I_{0}, \\
R_{1}=\mathscr{K}_{C, U}\left\{F_{i, j}\right\}_{\partial_{z}, \Delta_{t}}=\mathscr{K}_{C, U}\left\{Y_{i, j}\right\}_{\partial_{z}, \Delta_{t}} / I_{1} .
\end{gathered}
$$

The group $\operatorname{Aut}_{\partial_{z}}^{\Delta_{t}}\left(\left(K_{U}\right)^{\sim} \mid K_{U}\right)$ (resp. $\left.\operatorname{Gal}_{\partial_{z}}^{\Delta_{t}}\left(\left(\mathscr{K}_{C, U}\right)^{\sim} \mid \mathscr{K}_{C, U}\right)\right)$ is the set of $B \in$ $\mathrm{GL}_{m}\left(M_{U}\right)$ (resp. $\left.B \in \mathrm{GL}_{m}(C)\right)$ such that $\left(F_{i, j}\right) B$ is again a zero of $I_{0}$ (resp. $I_{1}$ ). We just have to prove that $I_{1}=C I_{0}$. The inclusion $C I_{0} \subset I_{1}$ is clear. Let us prove the other inclusion. Let $P \in I_{1}$. Without loss of generality, we may assume that $P \in\left(K_{U} \otimes_{\mu_{U}} C\right)\left[Y_{i, j}\right]$. Let us write $P=\sum d_{k} P_{k}$, where $P_{k} \in K_{U}\left[Y_{i, j}\right]$. One finds that

$$
P\left(F_{i, j}\right)=\sum d_{k} P_{k}\left(F_{i, j}\right)=0 .
$$


Since the $d_{k}$ are linearly independent over $\left(K_{U}\right)^{\sim}$, one finds that $P_{k}\left(F_{i, j}\right)=0$, and therefore $I_{1}=C I_{0}$.

2D. An analogue of the density theorem in the parametrized case. Let us consider the equation (*), with $A(z, t) \in \mathrm{M}_{m}\left(\mathrm{O}_{U}(\{z\})\right)$, where $U$ is a nonempty polydisc in $\mathbb{C}^{n}$. We want to find topological generators for Aut $\partial_{\partial_{z}}^{\Delta_{t}}\left(\left(K_{U}\right)^{\sim} \mid K_{U}\right)$ for the Kolchin topology.

We now define the parametrized monodromy. The notion of monodromy in the unparametrized case is well explained in [van der Put and Singer 2003]. For more details about parametrized monodromy, see [Cassidy and Singer 2007; Mitschi and Singer 2012; 2013; Sibuya 1990].

Definition 2.13. The notations are introduced in Section 1A. We define $\hat{m}$, the formal parametrized monodromy, as follows:

- $\hat{m}(\hat{H}(z, t))=\hat{H}(z, t)$ for all $\hat{H}(z, t) \in \hat{K}_{U}$.

- $\hat{m}\left(z^{a(t)}\right)=e^{2 i \pi a(t)} z^{a(t)}$ for all $a(t) \in M_{U}$.

- $\hat{m}(\log )=2 i \pi+\log$.

- For all $q(z, t)=\sum a_{n} z^{-n} \in \mathbf{E}_{U}=\bigcup_{v \in \mathbb{Q}^{>0}} z^{-1 / v} \mathcal{M}_{U}\left[z^{-1 / \nu}\right]$, we define

$$
\hat{m}(e(q(z, t)))=e\left(\sum a_{n} e^{-2 i \pi n} z^{-n}\right) \text {. }
$$

From the construction of $\hat{K}_{U}\left[\log ,\left(z^{a(t)}\right)_{a(t) \in \mathcal{M}_{U}}(e(q(z, t)))_{q(z, t) \in \mathbf{E}_{U}}\right]$, it is easy to check that $\hat{m}$ induces a well defined $\left(\partial_{z}, \Delta_{t}\right)$-differential ring automorphism of $\hat{K}_{U}\left[\log ,\left(z^{a(t)}\right)_{a(t) \in \mathcal{M}_{U}}(e(q(z, t)))_{q(z, t) \in \mathbf{E}_{U}}\right]$, and then it can be extended as a $\left(\partial_{z}, \Delta_{t}\right)$-differential field automorphism of $\left(\mathbf{K}_{U}\right)^{\wedge}$ keeping $K_{U}$ invariant. Since $\left(K_{U}\right)^{\sim} \subset\left(\mathbf{K}_{U}\right)^{\wedge}$, and since $\left(K_{U}\right)^{\sim}$ is stable under $\hat{m}, \hat{m}$ induces an element of $\operatorname{Aut}_{\partial_{z}}^{\Delta_{t}}\left(\left(K_{U}\right)^{\sim} \mid K_{U}\right)$.

Remark 2.14. In the regular singular case with one singularity at 0 , the definition of formal parametrized monodromy restricts to the definition given in [Mitschi and Singer 2012].

We now introduce the parametrized exponential torus, which is a subgroup of $\operatorname{Aut}_{\partial_{z}}^{\Delta_{t}}\left(\left(K_{U}\right)^{\sim} \mid K_{U}\right)$ consisting of elements that act on the $e(q(z, t))$ with $q(z, t) \in \mathbf{E}_{U}$.

Definition 2.15. Let $\alpha$ be a character of $\mathbf{E}_{U}$. We define $\tau_{\alpha}$ as follows:

- $\tau_{\alpha}$ is the identity on $\hat{K}_{F, U}$.

- $\tau_{\alpha}(e(q(z, t)))=\alpha(q(z, t)) e(q(z, t))$ for all $q(z, t) \in \mathbf{E}_{U}$.

From the construction of $\hat{K}_{U}\left[\log ,\left(z^{a(t)}\right)_{a(t) \in \mathcal{M}_{U}}(e(q(z, t)))_{q(z, t) \in \mathbf{E}_{U}}\right]$, it is easy to check that $\tau_{\alpha}$ induces a well defined $\left(\partial_{z}, \Delta_{t}\right)$-differential ring automorphism of $\hat{K}_{U}\left[\log ,\left(z^{a(t)}\right)_{a(t) \in \mathcal{M}_{U}}(e(q(z, t)))_{q(z, t) \in \mathbf{E}_{U}}\right]$, and then it can be extended to a $\left(\partial_{z}, \Delta_{t}\right)$ differential field automorphism of $\left(\mathbf{K}_{U}\right)^{\wedge}$ keeping $K_{U}$ invariant. Since $\left(K_{U}\right)^{\sim} \subset$ 
$\left(\mathbf{K}_{U}\right)^{\wedge}$, and since $\left(K_{U}\right)^{\sim}$ is stable under $\tau_{\alpha}$, the map $\tau_{\alpha}$ induces an element of $\operatorname{Aut}_{\partial_{z}}^{\Delta_{t}}\left(\left(K_{U}\right)^{\sim} \mid K_{U}\right)$.

The parametrized exponential torus (or simply, the exponential torus) is the subgroup of $\operatorname{Aut}_{\partial_{z}}^{\Delta_{t}}\left(\left(K_{U}\right)^{\sim} \mid K_{U}\right)$ consisting of the $\tau_{\alpha}$, where $\alpha$ is a character of $\mathbf{E}_{U}$. Notice that the matrices of the exponential torus belongs to $\mathrm{GL}_{m}(\mathbb{C})$, while the coefficients of the matrix of $\hat{m}$ depend upon $t$.

Example 2.16. Let $t=\left(t_{1}, t_{2}\right)$ and let us consider

$$
\partial_{z}\left(\begin{array}{l}
Y_{1}(z, t) \\
Y_{2}(z, t)
\end{array}\right)=\left(\begin{array}{cc}
-t_{1} z^{-2} & 0 \\
0 & -t_{2} z^{-2}
\end{array}\right)\left(\begin{array}{l}
Y_{1}(z, t) \\
Y_{2}(z, t)
\end{array}\right)
$$

which admits $\left(\begin{array}{cc}e^{t_{1} / z} & 0 \\ 0 & e^{t_{2} / z}\end{array}\right)$ as fundamental solution. The parametrized exponential torus and the parametrized differential Galois group are both equal to

$$
\left\{\left(\begin{array}{ll}
\alpha & 0 \\
0 & \beta
\end{array}\right) \mid \alpha, \beta \in \mathbb{C}^{*}\right\}
$$

Remark that the unparametrized exponential torus (see p. 80 of [van der Put and Singer 2003]) and the unparametrized differential Galois group are isomorphic to $\left(\mathbb{C}^{*}\right)^{2}$ if and only if $t_{1}$ and $t_{2}$ are linearly independent over $\mathbb{Q}$. In particular, the matrices of the parametrized exponential torus evaluated at a specialized value $(u, v)$ of the parameter are not always equal to the matrices of the unparametrized exponential torus of the system

$$
\partial_{z}\left(\begin{array}{l}
Y_{1}(z, u, v) \\
Y_{2}(z, u, v)
\end{array}\right)=\left(\begin{array}{cc}
-u z^{-2} & 0 \\
0 & -v z^{-2}
\end{array}\right)\left(\begin{array}{l}
Y_{1}(z, u, v) \\
Y_{2}(z, u, v)
\end{array}\right) .
$$

This is a difference between the exponential torus and the two other generators of the parametrized differential Galois group: the monodromy and the Stokes operators (see Definition 2.18 below).

Lemma 2.17. Let $d(t)$ be a singular direction of $(*)$ (see Section 1D). The Stokes matrix $\mathrm{St}_{d(t)}$ induces an element of $\operatorname{Aut}_{\partial_{z}}^{\Delta_{t}}\left(\left(K_{U}\right)^{\sim} \mid K_{U}\right)$.

Proof. Let us recall the construction of the Stokes matrices. Let $d(t)$ be a singular direction and let $k_{r}$ be the biggest level of $(*)$. The assumption we have made on $\mathscr{D}$ (see Section 1D) tells us that there exists $t \mapsto d^{ \pm}(t)$, continuous in $t$, such that

$$
d(t)-\frac{\pi}{2 k_{r}}<d^{-}(t)<d(t)<d^{+}(t)<d(t)+\frac{\pi}{2 k_{r}},
$$

with no singular directions in $\left[d^{-}(t), d(t)[\cup] d(t), d^{+}(t)\right]$. From the construction of $\mathrm{St}_{d(t)}$, and Section 1C, we know that

$$
H^{d^{+}(t)}(z, t) e^{L(t) \log (z)} e^{Q(z, t)}=H^{d^{-}(t)}(z) e^{L(t) \log (z)} e^{Q(z, t)} \mathrm{St}_{d(t)} .
$$


By construction, the Stokes matrix induces the identity on $K_{U}$. To prove that the Stokes matrices are elements of $\operatorname{Aut}_{\partial_{z}}^{\Delta_{t}}\left(\left(K_{U}\right)^{\sim} \mid K_{U}\right)$, we have to prove that the maps $i^{ \pm}$that send $\hat{H}(z, t) z^{L(t)} e(Q(z, t))$ to $H^{d^{ \pm}(t)}(z, t) e^{L(t) \log (z)} e^{Q(z, t)}$ induce $\left(\partial_{z}, \Delta_{t}\right)$-field isomorphisms. From the unparametrized case (see Theorem 2, $\$ 6.4$ of [Balser 1994]), and the relations satisfied by the symbols $\log ,\left(z^{a(t)}\right)_{a(t) \in \mathcal{M}_{U}}$ and $(e(q(z, t)))_{q(z, t) \in \mathbf{E}_{U}}$ (see Section 1A), $i^{ \pm}$induce $\partial_{z}$-field isomorphisms.

We want now to prove that if $\hat{H}(z, t)$ admits $H^{d^{ \pm}(t)}(z, t)$ as asymptotic sum in the direction $d^{ \pm}(t)$, then $\partial_{t_{i}} \hat{H}(z, t)$ admits $\partial_{t_{i}} H^{d^{ \pm}(t)}(z, t)$ as asymptotic sum in the direction $d^{ \pm}(t)$ for all $i \leq n$. This is a consequence of Lemma 1.14 and the fact that we may assume that the $d^{ \pm}(t)$ are locally constant. Hence $i^{ \pm}$commute with $\partial_{t_{i}}$, and $i^{ \pm}$induce $\left(\partial_{z}, \Delta_{t}\right)$-field isomorphisms.

Definition 2.18. Let $d(t)$ be a singular direction of $(*)$. Then the element of $\operatorname{Aut}_{\partial_{z}}^{\Delta_{t}}\left(\left(K_{U}\right)^{\sim} \mid K_{U}\right)$ induced by the Stokes matrix in the direction $d(t)$ is the Stokes operator in the direction $d(t)$. For simplicity of notation, we write $\mathrm{St}_{d(t)}$ for both the Stokes operator and the Stokes matrix in the direction $d(t)$.

Proposition 2.19. If $g(z, t) \in\left(K_{U}\right)^{\sim}$ is fixed by all the Stokes operators $\mathrm{St}_{d(t)}$, the monodromy and the exponential torus, then $g(z, t) \in K_{U}$.

Proof. Let $\bar{M}_{U}$ be the algebraic closure of $\mu_{U}$. Proposition 3.25 of [van der Put and Singer 2003] implies that if $g(z, t) \in\left(\mathbf{K}_{U}\right)^{\wedge}$ is fixed by the monodromy and the exponential torus, then $g(z, t) \in\left(\mathbf{K}_{U}\right)^{\wedge} \cap \bar{M}_{U} \llbracket z \rrbracket\left[z^{-1}\right]=\hat{K}_{U}$. Since $\left(K_{U}\right)^{\sim} \subset\left(\mathbf{K}_{U}\right)^{\wedge}$, we have to prove that if $g(z, t) \in\left(K_{U}\right)^{\sim} \cap \hat{K}_{U}$ is fixed by all the Stokes operators, then $g(z, t) \in K_{U}$. Let $g(z, t) \in\left(K_{U}\right)^{\sim} \cap \hat{K}_{U}$ be fixed by all the Stokes operators. Let $F(z, t)=\hat{H}(z, t) z^{L(t)} e(Q(z, t))$ be the fundamental solution defined in Proposition 1.3, and let $\left(\hat{H}_{i, j}\right)$ be the entries of the matrix $\hat{H}(z, t)$. There exists $P \in K_{U}\left\langle X_{i, j}\right\rangle_{\partial_{z}, \Delta_{t}}$ such that $P\left(\hat{H}_{i, j}\right)=g(z, t)$. Let $d(t)$ satisfy the same properties as in Proposition 1.13. Because of Proposition 1.13, there exists a map $U \rightarrow \mathbb{R}^{>0}, t \mapsto \varepsilon(t)$ (which is not necessarily continuous) such that $P\left(H_{i, j}^{d(t)}\right)$ is meromorphic in $(z, t)$ for

$$
z \in \Sigma\left(d_{1}(t)-\pi / 2 k_{r}, d_{2}(t)+\pi / 2 k_{r}\right) \text { with } 0<|z|<\varepsilon(t) \quad \text { and } \quad t \in U,
$$

where $d_{1}(t), d_{2}(t)$ are two singular directions. Since $P\left(\hat{H}_{i, j}\right)$ is fixed by all the Stokes operators, $P\left(H_{i, j}^{d(t)}\right)$ is meromorphic in $(z, t)$ for $0<|z|<\varepsilon(t)$ and $(z, t) \in \widetilde{\mathbb{C}} \times U$. Moreover, $P\left(H_{i, j}^{d(t)}\right)(z, t)=P\left(H_{i, j}^{d(t)}\right)\left(e^{2 i \pi} z, t\right)$ on its domain of definition, which means that $P\left(H_{i, j}^{d(t)}\right)$ is meromorphic in $(z, t)$ for $0<|z|<\varepsilon(t)$ and $(z, t) \in \mathbb{C} \times U$. We recall that $K_{U}$ consists of elements $f(z, t) \in \hat{K}_{U}$ such that for $0<|z|<\varepsilon(t)$, the function $t \mapsto f(z, t)$ lies in $M_{U}$. We have $P\left(H_{i, j}^{d(t)}\right) \in K_{U}$. We have seen in Lemma 2.17 that the map that sends $\hat{H}(z, t) z^{L(t)} e(Q(z, t))$ to $H^{d(t)}(z, t) e^{L(t) \log (z)} e^{Q(z, t)}$ induces a $\left(\partial_{z}, \Delta_{t}\right)$-field isomorphism. Since this map leaves $K_{U}$ invariant, this implies that $P\left(\hat{H}_{i, j}\right)=g(z, t) \in K_{U}$. 
We can now prove the main theorem of this paper. We recall some notation. Let Consider the equation (*) with $A(z, t) \in \mathrm{M}_{m}\left(\mathcal{O}_{U}(\{z\})\right)$ (see page 90$)$, let $K_{U}$ be the fraction field of $\mathcal{O}_{U}(\{z\})$, and let $\left(K_{U}\right)^{\sim} \mid K_{U}$ be the parametrized Picard-Vessiot extension defined in the beginning of Section 2D. Let $\operatorname{Aut}_{\partial_{z}}^{\Delta_{t}}\left(\left(K_{U}\right)^{\sim} \mid K_{U}\right)$ be the field automorphisms of $\left(K_{U}\right)^{\sim}$ which commute with all the derivations and leave $K_{U}$ invariant.

Theorem 2.20 (parametrized analogue of the density theorem of Ramis). The group generated by the monodromy, the exponential torus and the Stokes operators is dense for the Kolchin topology in $\operatorname{Aut}_{\partial_{z}}^{\Delta_{t}}\left(\left(K_{U}\right)^{\sim} \mid K_{U}\right)$.

Proof. First of all, we have already pointed out that the monodromy, the exponential torus and the Stokes operators are elements of $\operatorname{Aut}_{\partial_{z}}^{\Delta_{t}}\left(\left(K_{U}\right)^{\sim} \mid K_{U}\right)$. Using Proposition 2.9, we just have to prove that if $\alpha(z, t) \in\left(K_{U}\right)^{\sim}$ is fixed by the monodromy, the exponential torus and the Stokes operators, then it belongs to $K_{U}$. This is exactly Proposition 2.19.

Remark 2.21. (1) Let $\mathbb{C}(t)\{z\}$ be the subset of $\mathrm{O}_{U}(\{z\})$ consisting of elements of the form $\sum_{i>N} a_{i}(t) z^{i}$, with $a_{i}(t) \in \mathbb{C}(t)$ and $N \in \mathbb{Z}$. Let us consider the equation (*) with $A(z, t) \in \mathrm{M}_{m}(\mathbb{C}(t)\{z\}) p$. Even if we were able to define a parametrized PicardVessiot extension over $\mathbb{C}(t)\{z\}$, we would not have a parametrized analogue of the density theorem of Ramis, because the monodromy is not defined in this case. In general, we have

$$
\hat{m}\left(z^{\alpha(t)}\right)=e^{2 i \pi \alpha(t)} z^{\alpha(t)} \notin \mathbb{C}(t)\{z\}\left(z^{\alpha(t)}\right) .
$$

This is why we take a larger field of constants with respect to $\partial_{z}$.

(2) Similarly, we can prove that the group generated by the monodromy and the exponential torus is dense for the Kolchin topology in $\operatorname{Aut}_{\partial_{z}}^{\Delta_{t}}\left(\left(K_{U}\right)^{\sim} \mid \hat{K}_{U} \cap\left(K_{U}\right)^{\sim}\right)$.

Corollary 2.22. Aut $_{\partial_{z}}^{\Delta_{t}}\left(\left(K_{U}\right)^{\sim} \mid K_{U}\right)$ contains a finitely generated Kolchin-dense subgroup.

Proof. Let $q_{1}(z, t), \ldots, q_{\beta}(z, t) \in \mathbf{E}_{U}$ be $\mathbb{Q}$-linearly independent such that

$$
\left(K_{U}\right)^{\sim} \subset \hat{K}_{F, U}\left(e\left(q_{1}(z, t)\right), \ldots, e\left(q_{\beta}(z, t)\right)\right) .
$$

Let $\tau_{i}$ be an element of the exponential torus that fixes the $e\left(q_{j}(z, t)\right)$ for $j \neq i$, and that sends $e\left(q_{i}(z, t)\right)$ to $a e\left(q_{i}(z, t)\right)$, with $a$ not a root of unity.

By definition of the singular directions (see Section 1D), there exists $v \in \mathbb{N}^{*}$ such that there are finitely many singular directions modulo $2 v \pi$. Let $d_{1}(t), \ldots, d_{k}(t)$ be continuous singular directions such that, if $d(t)$ is a singular direction, then $d(t)$ is equal to one of the $d_{i}(t)$ modulo $2 v \pi$. Let $g(z, t) \in\left(K_{U}\right)^{\sim}$ be fixed by the monodromy, $\tau_{1}, \ldots, \tau_{\beta}$, and $\mathrm{St}_{d_{1}(t)}, \ldots, \mathrm{St}_{d_{k}(t)}$. Using Proposition 2.9(2), it is sufficient to prove that $g(z, t) \in K_{U}$. 
We can write $g(z, t)$ as an element of

$$
\hat{K}_{F, U}\left(e\left(q_{1}(z, t)\right), \ldots, e\left(q_{\beta-1}(z, t)\right)\right)\left(e\left(q_{\beta}(z, t)\right)\right) .
$$

Since the $q_{i}(z, t) \in \mathbf{E}_{U}$ are $\mathbb{Q}$-linearly independent, we know by construction that the $e\left(N q_{\beta}(z, t)\right)$, with $N \in \mathbb{Z}$, are $\mathbb{C}$-linearly independent over

$$
\hat{K}_{F, U}\left(e\left(q_{1}(z, t)\right), \ldots, e\left(q_{\beta-1}(z, t)\right)\right) .
$$

If we add the fact that $g(z, t)$ is fixed by $\tau_{\beta}$, we obtain

$$
g(z, t) \in \hat{K}_{F, U}\left(e\left(q_{1}(z, t)\right), \ldots, e\left(q_{\beta-1}(z, t)\right)\right) .
$$

We apply the same argument $\beta$ times to conclude that $g(z, t) \in \hat{K}_{F, U} \cap\left(K_{U}\right)^{\sim}$. By the construction of the Stokes operators, we have that $\mathrm{St}_{d(t)}=\mathrm{Id}$ if and only if $\mathrm{St}_{2 v \pi+d(t)}=\mathrm{Id}$, where $v \in \mathbb{N}^{*}$ has been defined in the proof. Proposition 2.19 allows us to conclude that $g(z, t) \in K_{U}$.

\section{E. The density theorem for the global parametrized differential Galois group.}

In this subsection, we consider parametrized linear differential equations of the form (*), with $A(z, t) \in \mathrm{M}_{m}\left(M_{U}(z)\right)$. We want to prove a density theorem for the global parametrized differential Galois group. The result in the unparametrized case is due to Ramis, and a proof can be found for instance in [Mitschi 1996, Proposition 1.3]. The parametrized singularities of (*) (that is, the poles of $A(z, t)$ as a rational function in $z$, possibly including $\infty$ ) belong to the algebraic closure of $\mathcal{M}_{U}$. Because of Remark 1.1, after taking a smaller nonempty polydisc $U$, we may assume that the set of parametrized singularities belongs to $\mathcal{M}_{U}$. We will write "singularity" instead of "parametrized singularity" when no confusion is likely to arise. Let $S=\left\{\alpha_{1}(t), \ldots, \alpha_{k}(t)\right\} \subset \mathbb{P}_{1}\left(\mathcal{M}_{U}\right)$ be the set of the singularities of $(*)$. For any singularity $\alpha(t)$ of this equation, we may define its levels and its set of singular directions by considering

$$
\partial_{z} Y(z-\alpha(t), t)=A(z-\alpha(t), t) Y(z-\alpha(t), t) \quad \text { if } \infty \not \equiv \alpha(t) \in S
$$

and

$$
\partial_{z} Y\left(z^{-1}, t\right)=A\left(z^{-1}, t\right) Y\left(z^{-1}, t\right) \quad \text { if } \infty \equiv \alpha(t) \in S .
$$

Let $\left(d_{i, j}(t)\right)$ be the singular directions of $\alpha_{i}(t)$. As in Section 1D, we define $\mathscr{D}_{\alpha_{i}(t)}=\left\{t \in U \mid\right.$ there exist $j, j^{\prime} \in \mathbb{N}$ such that $d_{i, j} \not \equiv d_{i, j^{\prime}}$ and $\left.d_{i, j}(t)=d_{i, j^{\prime}}(t)\right\}$.

From Lemma 1.12, all the $\mathscr{D}_{\alpha_{i}(t)}$ are closed sets with empty interior. After taking a smaller nonempty polydisc $U$, we may assume that:

- There exists $\varepsilon>0$ such that for all $t \in U$ and for all $i \neq j$,

$$
\left|\alpha_{i}(t)-\alpha_{j}(t)\right|>\varepsilon
$$


- $\mathscr{D}_{\alpha_{i}(t)}=\varnothing$ for all $i \leq k$.

- For all singularities of $(*)$, the levels are independent of $t$.

- For all $t_{0} \in U$ and all singularities $\infty \not \equiv \alpha(t) \in S$, the singular directions of the equation $\partial_{z} Y(z-\alpha(t), t)=A(z-\alpha(t), t) Y(z-\alpha(t), t)$ evaluated at $t_{0}$ are equal to the singular directions of the specialized system $\partial_{z} Y\left(z-\alpha(t), t_{0}\right)=$ $A\left(z-\alpha(t), t_{0}\right) Y\left(z-\alpha(t), t_{0}\right)$.

- Similarly, for all $t_{0} \in U$, the singular directions of the equation $\partial_{z} Y\left(z^{-1}, t_{0}\right)=$ $A\left(z^{-1}, t_{0}\right) Y\left(z^{-1}, t_{0}\right)$ evaluated at $t_{0}$ are equal to the singular directions of the specialized system $\partial_{z} Y\left(z^{-1}, t_{0}\right)=A\left(z^{-1}, t_{0}\right) Y\left(z^{-1}, t_{0}\right)$.

- Every entry of every $z$-coefficient of $A(z, t)$ is analytic on $U$.

Let $x_{0}(t) \in M_{U}$ and let $\varepsilon>0$ such that

$$
\left|x_{0}(t)-\alpha_{j}(t)\right|>\varepsilon \quad \text { and } \quad\left|\alpha_{i}(t)-\alpha_{j}(t)\right|>\varepsilon \quad \text { for all } t \in U, i<j \leq k .
$$

For all $i \leq k$ and all $t \in U$, we define $U_{\alpha_{i}(t)}$, the polydisc in the $z$-plane with center $\alpha_{i}(t)$ and with radius $\varepsilon$. Let $d_{\alpha_{i}}(t)$ be a continuous ray from $\alpha_{i}(t)$ in $U_{\alpha_{i}(t)}$, let $b_{\alpha_{i}}(t)$ be the continuous point of $d_{\alpha_{i}}(t)$ with $\left|b_{\alpha_{i}}(t)-\alpha_{i}(t)\right|=\varepsilon$, and let $\gamma_{\alpha_{i}}(t)$ be a continuous path in $\mathbb{P}_{1}\left(M_{U}\right)$ from $x_{0}(t)$ to $b_{\alpha_{i}}(t)$ such that $\left|\gamma_{\alpha_{i}}(t)-\alpha_{j}(t)\right|>\varepsilon / 2$ for all $t \in U$ and all $j \leq k$. Analytic continuation of $F(z, t)=\left(F_{i, j}\right)$, that is, a germ of solution at $x_{0}(t)$ with the path $\gamma_{\alpha_{i}}(t)$ and $d_{\alpha_{i}}(t)$, provides a fundamental solution $F^{d_{\alpha_{i}}(t)}(z, t)$ on a germ of open sector with vertex $\alpha_{i}(t)$ bisected by $d_{\alpha_{i}}(t)$.

Let $\left(M_{U}(z)\right)^{\sim}=M_{U}(X)\left\langle F_{i, j}\right\rangle_{\partial_{z}, \Delta_{t}}$. From the assumptions we have made on $x_{0}(t)$, we deduce that this field has a field of constants with respect to $\partial_{z}$ equal to $M_{U}$. Therefore, we deduce that $\left(\mathcal{M}_{U}(z)\right)^{\sim} \mid \mathcal{M}_{U}(z)$ is a parametrized Picard-Vessiot extension. The results of Section 2B may be applied here and we can define a parametrized differential Galois group $\operatorname{Aut}_{\partial_{z}}^{\Delta_{t}}\left(\left(\mathcal{M}_{U}(z)\right)^{\sim} \mid \mathcal{M}_{U}(z)\right)$, which will be identified with a linear differential algebraic subgroup of $\mathrm{GL}_{m}\left(M_{U}\right)$. We will make the same abuse of language as in the local case (see Remark 2.8) and call it the parametrized linear differential Galois group, or Galois group, if no confusion is likely to arise. As in Proposition 2.12, we want to prove now that it is the "same" as the one of Section 2A.

Let $C$ be a $\left(\Delta_{t}\right)$-differentially closed field that contains $\mathcal{M}_{U}$, and let $C(z)$ denote the $\left(\partial_{z}, \Delta_{t}\right)$-differential field of rational functions in the indeterminate $z$ with coefficients in $C$, where $z$ is a $\left(\Delta_{t}\right)$-constant with $\partial_{z} z=1, C$ is the field of constants with respect to $\partial_{z}$, and $\partial_{z}$ commutes with all the derivations. The next proposition is the analogue in the global case of Proposition 2.12.

Proposition 2.23. Let us keep the same notation. Consider the equation $\partial_{z} Y(z, t)=$ $A(z, t) Y(z, t)$, with $A(z, t) \in \mathrm{M}_{m}\left(M_{U}(z)\right)$. The extension field

$$
C(z)\left\langle F_{i, j}\right\rangle_{\partial_{z}, \Delta_{t}}\left|C(z):=(C(z))^{\sim}\right| C(z)
$$


is a parametrized Picard-Vessiot extension for $\partial_{z} Y(z, t)=A(z, t) Y(z, t)$. Moreover, there exist $P_{1}, \ldots, P_{k} \in \mathcal{M}_{U}\left\{X_{i, j}\right\}_{\Delta_{t}}$ such that the image of the representation of $\operatorname{Gal}_{\partial_{z}}^{\Delta_{t}}\left((C(z))^{\sim} \mid C(z)\right)$ (resp. Aut $\left.\partial_{z} \Delta_{t}\left(\left(M_{U}(z)\right)^{\sim} \mid \mathcal{M}_{U}(z)\right)\right)$ associated to $F(z, t)$ is the set of $C$-rational points (resp. $M_{U}$-rational points) of the linear differential algebraic subgroup of $\mathrm{GL}_{m}(C)$ (resp. $\mathrm{GL}_{m}\left(M_{U}\right)$ ) defined by $P_{1}, \ldots, P_{k}$. More explicitly:

$$
\begin{aligned}
& \left\{F^{-1} \varphi(F) \mid \varphi \in \mathrm{Gal}_{\partial_{z}}^{\Delta_{t}}\left((C(z))^{\sim} \mid C(z)\right)\right\} \\
& =\left\{A=\left(a_{i, j}\right) \in \mathrm{GL}_{m}(C) \mid P_{1}\left(a_{i, j}\right)=\cdots=P_{k}\left(a_{i, j}\right)=0\right\}, \\
& \left\{F^{-1} \varphi(F) \mid \varphi \in \operatorname{Aut}_{\partial_{z}}^{\Delta_{t}}\left(\left(\mathcal{M}_{U}(z)\right)^{\sim} \mid \mathcal{M}_{U}(z)\right)\right\} \\
& =\left\{A=\left(a_{i, j}\right) \in \mathrm{GL}_{m}\left(M_{U}\right) \mid P_{1}\left(a_{i, j}\right)=\cdots=P_{k}\left(a_{i, j}\right)=0\right\} .
\end{aligned}
$$

Proof. This is exactly the same reasoning as in Proposition 2.12 .

We want to find topological generators for $\operatorname{Aut}_{\partial_{z}}^{\Delta_{t}}\left(\left(M_{U}(z)\right)^{\sim} \mid \mathcal{M}_{U}(z)\right)$ for the Kolchin topology.

For $\alpha(t) \in M_{U}$, let

$$
K_{U, \alpha(t)}=\left\{f(z-\alpha(t), t) \mid f(z, t) \in K_{U}\right\}
$$

and let

$$
K_{U, \infty}=\left\{f\left(z^{-1}, t\right) \mid f(z, t) \in K_{U}\right\} .
$$

Let $\alpha(t) \in S$ and let $\operatorname{Aut}_{\partial_{z}}^{\Delta_{t}}\left(\left(M_{U}(z)\right)^{\sim} \mid K_{U, \alpha(t)} \cap\left(M_{U}(z)\right)^{\sim}\right)$ be the local Galois group for the fundamental solution $F^{d_{\alpha}(t)}(z, t)$ described above. If we conjugate $\operatorname{Aut}_{\partial_{z}}^{\Delta_{t}}\left(\left(M_{U}(z)\right)^{\sim} \mid K_{U, \alpha(t)} \cap\left(M_{U}(z)\right)^{\sim}\right)$ by the differential isomorphism defined by analytic continuation of $F(z, t)$ described above, we get an injective morphism of linear differential algebraic groups

$$
\operatorname{Aut}_{\partial_{z}}^{\Delta_{t}}\left(\left(M_{U}(z)\right)^{\sim} \mid K_{U, \alpha(t)} \cap\left(M_{U}(z)\right)^{\sim}\right) \hookrightarrow \operatorname{Aut}_{\partial_{z}}^{\Delta_{t}}\left(\left(M_{U}(z)\right)^{\sim} \mid \mathcal{M}_{U}(z)\right) .
$$

Using the maps $i^{ \pm}$defined in the proof of Lemma 2.17 and the injection above, we can define the monodromy, the exponential torus and the Stokes operators for any singularities in $S$ as elements of

$$
\operatorname{Aut}_{\partial_{z}}^{\Delta_{t}}\left(\left(M_{U}(z)\right)^{\sim} \mid \mathcal{M}_{U}(z)\right) .
$$

Theorem 2.24 (global parametrized analogue of the density theorem of Ramis). Consider the equation $\partial_{z} Y(z, t)=A(z, t) Y(z, t)$, where $A(z, t) \in \mathrm{M}_{m}\left(M_{U}(z)\right)$. For $\alpha(t) \in S$, let $G_{\alpha(t)}$ be the subgroup of

$$
\operatorname{Aut}_{\partial_{z}}^{\Delta_{t}}\left(\left(M_{U}(z)\right)^{\sim} \mid K_{U, \alpha(t)} \cap\left(\mathcal{M}_{U}(z)\right)^{\sim}\right)
$$

generated by the monodromy, the exponential torus and the Stokes operators. Let $G$ be the subgroup of $\operatorname{Aut}_{\partial_{z}}^{\Delta_{t}}\left(\left(M_{U}(z)\right)^{\sim} \mid \mathcal{M}_{U}(z)\right)$ generated by the $G_{\alpha(t)}$ with $\alpha(t) \in S$. 
Then $G$ in dense for the Kolchin topology in

$$
\operatorname{Aut}_{\partial_{z}}^{\Delta_{t}}\left(\left(M_{U}(z)\right)^{\sim} \mid \mathcal{M}_{U}(z)\right) .
$$

Proof. We use (2) of Proposition 2.9. We have to prove that the subfield of $\left(M_{U}(z)\right)^{\sim}$ fixed by $G$ is $M_{U}(z)$. Let $f(z, t) \in\left(M_{U}(z)\right)^{\sim}$ be fixed by $G$. Then, by the same reasoning as in Proposition 2.19, it follows that $f(z, t)$ belongs to $K_{U, \alpha(t)}$ for $\alpha(t) \in S$. Therefore, we deduce that $f(z, t)$ is meromorphic in $(z, t)$ on $\mathbb{P}_{1}(\mathbb{C}) \times U$ and has a finite number of poles in the $z$-plane for $t$ fixed. Hence $f(z, t) \in \mathcal{M}_{U}(z)$.

In particular, this generalizes Theorem 4.2 in [Mitschi and Singer 2012], which says that if the equation has only regular singular poles, then the group generated by the monodromy at each pole is dense for the Kolchin topology in $\operatorname{Aut}_{\partial_{z}}^{\Delta_{t}}\left(\left(\mathcal{M}_{U}(z)\right)^{\sim} \mid \mathcal{M}_{U}(z)\right)$.

Corollary 2.25. $\operatorname{Aut}_{\partial_{z}}^{\Delta_{t}}\left(\left(M_{U}(z)\right)^{\sim} \mid \mathcal{M}_{U}(z)\right)$ contains a finitely generated Kolchindense subgroup.

Proof. In the proof of Theorem 2.24, we see that the global parametrized differential Galois group is generated by all local parametrized differential Galois groups. Since there is a finite number of singularities, this is a consequence of Corollary 2.22.

2F. Examples. In all the examples, we will compute the global parametrized differential Galois group. This means that the base field is $\mu_{U}(z)$.

Example 2.26. Let us consider the equation $\partial_{z} Y(z, t)=(t / z) Y(z, t)$. This example was considered by direct computations in Example 2.5, but we will compute here $\operatorname{Aut}_{\partial_{z}}^{\Delta_{t}}\left(\left(M_{U}(z)\right)^{\sim} \mid \mathcal{M}_{U}(z)\right)$ using the parametrized density theorem. The fundamental solution is $\left(z^{t}\right)$ and the parametrized Picard-Vessiot extension over $\mu_{U}(z)$ is $\mu_{U}\left(z, z^{t}, \log\right.$ ) (we want the extension to be closed under the derivations $\partial_{z}$ and $\partial_{t}$ ). The exponential torus and the Stokes matrices are trivial. The monodromy sends $z^{t}$ to $e^{2 i \pi t} z^{t}$. The element $e^{2 i \pi t}$ satisfies the differential equation

$$
\partial_{t}\left(\frac{\partial_{t} e^{2 i \pi t}}{e^{2 i \pi t}}\right)=0
$$

Therefore, the Kolchin closure of the monodromy is contained in

$$
\left\{a \in M_{U} \mid \partial_{t}\left(\frac{\partial_{t} a}{a}\right)\right\}=\left\{c e^{b t} \mid b \in \mathbb{C}, c \in \mathbb{C}^{*}\right\} .
$$

Conversely, the map $z^{t} \mapsto c e^{b t} z^{t}$ is an element of $\operatorname{Aut}_{\partial_{z}}^{\Delta_{t}}\left(\left(\mathcal{M}_{U}(z)\right)^{\sim} \mid \mathcal{M}_{U}(z)\right)$. Finally, 
viewed as a linear differential algebraic subgroup of $\mathrm{GL}_{1}\left(\mu_{U}\right)$,

$$
\begin{aligned}
\operatorname{Aut}_{\partial_{z}}^{\Delta_{t}}\left(\left(\mathcal{M}_{U}(z)\right)^{\sim} \mid \mathcal{M}_{U}(z)\right) & \simeq\left\{a \in \mathcal{M}_{U} \mid \partial_{t}\left(\frac{\partial_{t} a}{a}\right)=0\right\} \\
& =\left\{a \in \mathcal{M}_{U} \mid a \neq 0 \text { and } a \partial_{t}^{2} a-\left(\partial_{t} a\right)^{2}=0\right\} \\
& \subseteq \operatorname{GL}_{1}\left(\mathcal{M}_{U}\right) .
\end{aligned}
$$

Example 2.27 (parametrized Euler equation). Let $f(t)$ be an analytic function different from 0 , and let us consider the equation

$$
\partial_{z}^{2} Y(z, t)+\left(\frac{1}{z}-\frac{1}{f(t) z^{2}}\right) \partial_{z} Y(z, t)+\frac{1}{f(t) z^{3}} Y(z, t)=0,
$$

which can be seen as a system:

$$
\partial_{z}\left(\begin{array}{c}
Y(z, t) \\
\partial_{z} Y(z, t)
\end{array}\right)=\left(\begin{array}{cc}
0 & 1 \\
\frac{-1}{f(t) z^{3}} & \frac{1}{f(t) z^{2}}-\frac{1}{z}
\end{array}\right)\left(\begin{array}{c}
Y(z, t) \\
\partial_{z} Y(z, t)
\end{array}\right) .
$$

If $f \equiv 1$, we recognize the Euler equation. A fundamental solution is

$$
\left(\begin{array}{cc}
1 & \hat{F}(z, t) \\
\frac{1}{f(t) z^{2}} & \partial_{z} \hat{F}(z, t)
\end{array}\right)\left(\begin{array}{cc}
e\left(\frac{-1}{f(t) z}\right) & 0 \\
0 & 1
\end{array}\right)
$$

where $\hat{F}(z, t)=-\sum_{n \geq 0} n !(f(t) z)^{n+1}$. The only singularity is 0 . The monodromy is trivial. Let $\tau$ be an element of the exponential torus. Then the image of the fundamental solution under $\tau$ is

$$
\left(\begin{array}{cc}
1 & \hat{F}(z, t) \\
\frac{1}{f(t) z^{2}} & \partial_{z} \hat{F}(z, t)
\end{array}\right)\left(\begin{array}{cc}
\alpha e\left(\frac{-1}{f(t) z}\right) & 0 \\
0 & 1
\end{array}\right),
$$

with $\alpha \in \mathbb{C}^{*}$. Therefore, the matrices of the elements of the exponential torus are of the form $\operatorname{Diag}(\alpha, 1)$, with $\alpha \in \mathbb{C}^{*}$. The only level of the system is 1 and the singular directions are the $\arg \left(f(t)^{-1}\right)+2 k \pi$, with $k \in \mathbb{Z}$. As we have seen in Proposition 1.10, we can compute the Stokes matrix with the Laplace and the Borel transforms. It follows from the definition of the formal Borel transform that

$$
\hat{\mathscr{B}}_{1}(\hat{F}(z, t)) \equiv \log (1-f(t) z) .
$$

Let $0<\varepsilon<\pi / 2$ be such that there are no singular directions in

$$
\left[\arg \left(f(t)^{-1}\right)-\varepsilon, \arg \left(f(t)^{-1}\right)[\cup] \arg \left(f(t)^{-1}\right), \arg \left(f(t)^{-1}\right)+\varepsilon\right] .
$$

Then the following matrices are fundamental solutions:

$$
\left(\begin{array}{cc}
1 & \mathscr{L}_{1, \arg \left(f(t)^{-1}\right)+\varepsilon}(\log (1-f(t) z)) \\
\frac{1}{f(t) z^{2}} & \partial_{z} \mathscr{L}_{1, \arg \left(f(t)^{-1}\right)+\varepsilon}(\log (1-f(t) z))
\end{array}\right)\left(\begin{array}{cc}
e^{\frac{-1}{f(t) z}} & 0 \\
0 & 1
\end{array}\right),
$$




$$
\left(\begin{array}{cc}
1 & \mathscr{L}_{1, \arg \left(f(t)^{-1}\right)-\varepsilon}(\log (1-f(t) z)) \\
\frac{1}{f(t) z^{2}} & \partial_{z} \mathscr{L}_{1, \arg \left(f(t)^{-1}\right)-\varepsilon}(\log (1-f(t) z))
\end{array}\right)\left(\begin{array}{cc}
e^{\frac{-1}{f(t) z}} & 0 \\
0 & 1
\end{array}\right) .
$$

To compute the Stokes matrix in the direction $\arg \left(f(t)^{-1}\right)$, we have to compute

$$
\mathscr{L}_{1, \arg \left(f(t)^{-1}\right)+\varepsilon}(\log (1-f(t) z))-\mathscr{L}_{1, \arg \left(f(t)^{-1}\right)-\varepsilon}(\log (1-f(t) z)) .
$$

We have

$$
\begin{gathered}
\mathscr{L}_{1, \arg \left(f(t)^{-1}\right)+\varepsilon}(\log (1-f(t) z))-\mathscr{L}_{1, \arg \left(f(t)^{-1}\right)-\varepsilon}(\log (1-f(t) z)) \\
=z^{-1} \int_{0}^{\infty i\left(\arg \left(f(t)^{-1}\right)+\varepsilon\right)} \log (1-f(t) u) e^{-u / z} d(u) \\
-z^{-1} \int_{0}^{\infty i\left(\arg \left(f(t)^{-1}\right)-\varepsilon\right)} \log (1-f(t) u) e^{-u / z} d(u) .
\end{gathered}
$$

Integration by parts and the residue theorem imply that $\mathscr{L}_{1, \arg \left(f(t)^{-1}\right)+\varepsilon}(\log (1-f(t) z))-\mathscr{L}_{1, \arg \left(f(t)^{-1}\right)-\varepsilon}(\log (1-f(t) z))=2 i \pi f(t) e^{-\frac{1}{f(t) z}}$. Therefore, the Stokes matrix in this direction is $\left(\begin{array}{ccc}1 & 2 i \pi f(t) \\ 0 & 1\end{array}\right)$. Finally, we obtain

$$
\begin{aligned}
\operatorname{Aut}_{\partial_{z}}^{\Delta_{t}}\left(\left(M_{U}(z)\right)^{\sim} \mid \mathcal{M}_{U}(z)\right) & \simeq\left\{\left(\begin{array}{cc}
\alpha & b f \\
0 & 1
\end{array}\right) \mid \alpha \in \mathbb{C}^{*} \text { and } b \in \mathbb{C}\right\} \\
& \simeq\left\{\left(\begin{array}{cc}
\alpha & \beta \\
0 & 1
\end{array}\right) \mid \partial_{t} \alpha=0, \alpha \neq 0 \text { and } \partial_{t}\left(\frac{\beta}{f}\right)=0\right\} .
\end{aligned}
$$

Example 2.28 (Bessel equation). We are interested in the parametrized linear differential equation

$$
\partial_{z}\left(\begin{array}{c}
Y(z, t) \\
\partial_{z} Y(z, t)
\end{array}\right)=\left(\begin{array}{cc}
0 & 1 \\
\frac{t^{2}-z^{2}}{z^{2}} & \frac{-1}{z}
\end{array}\right)\left(\begin{array}{c}
Y(z, t) \\
\partial_{z} Y(z, t)
\end{array}\right) .
$$

This equation has two singularities: 0 and $\infty$. Let $U$ be a nonempty disc such that $U \cap(1 / 2+\mathbb{Z})=\varnothing$. First we will compute the local group at 0,

$$
\operatorname{Aut}_{\partial_{z}}^{\Delta_{t}}\left(\left(M_{U}(z)\right)^{\sim} \mid K_{U, 0} \cap\left(\mathcal{M}_{U}(z)\right)^{\sim}\right) .
$$

If $t+1 / 2 \notin \mathbb{Z}$, the two solutions

$$
\begin{gathered}
J_{t}(z)=\left(\frac{z}{2}\right)^{t} \sum_{k=0}^{\infty} \frac{(-1)^{k} z^{2 k}}{k ! \Gamma(t+k+1) 2^{k}}, \\
J_{-t}(z)=\left(\frac{z}{2}\right)^{-t} \sum_{k=0}^{\infty} \frac{(-1)^{k} z^{2 k}}{k ! \Gamma(-t+k+1) 2^{k}},
\end{gathered}
$$


are linearly independent (see [Watson 1944, p. 43]) and we have a fundamental solution of the specialized system. The equation is regular singular at $z=0$, and therefore the group generated by the monodromy $\hat{m}$ is dense for the Kolchin topology in the parametrized differential Galois group $\operatorname{Aut}_{\partial_{z}}^{\Delta_{t}}\left(\left(\mathcal{M}_{U}(z)\right)^{\sim} \mid K_{U, 0} \cap\left(\mathcal{M}_{U}(z)\right)^{\sim}\right)$. By the same reasoning as in Example 2.26,

$$
\operatorname{Aut}_{\partial_{z}}^{\Delta_{t}}\left(\left(M_{U}(z)\right)^{\sim} \mid K_{U, 0} \cap\left(M_{U}(z)\right)^{\sim}\right) \simeq\left\{\left(\begin{array}{cc}
\alpha & 0 \\
0 & \alpha^{-1}
\end{array}\right) \mid \alpha \neq 0, \alpha \partial_{t}^{2} \alpha-\left(\partial_{t} \alpha\right)^{2}=0\right\} .
$$

We now turn to the singularity at infinity. We have

$$
\partial_{z}\left(\begin{array}{c}
Y\left(z^{-1}, t\right) \\
\partial_{z} Y\left(z^{-1}, t\right)
\end{array}\right)=\left(\begin{array}{cc}
0 & 1 \\
\frac{t^{2}}{z^{2}}-\frac{1}{z^{4}} & \frac{-1}{z}
\end{array}\right)\left(\begin{array}{c}
Y\left(z^{-1}, t\right) \\
\partial_{z} Y\left(z^{-1}, t\right)
\end{array}\right) .
$$

In order to compute the matrices of the monodromy, the elements of the exponential torus and the Stokes operators, we make use of another basis of solutions:

$$
\begin{aligned}
H_{t}^{(1)}\left(z^{-1}\right) & =\frac{J_{-t}\left(z^{-1}\right)-e^{-i t \pi} J_{t}\left(z^{-1}\right)}{i \sin (t \pi)}, \\
H_{t}^{(2)}\left(z^{-1}\right) & =\frac{J_{-t}\left(z^{-1}\right)-e^{i t \pi} J_{t}\left(z^{-1}\right)}{-i \sin (t \pi)} .
\end{aligned}
$$

In [Watson 1944, p. 198], we find that on the sector ] $-\pi, 2 \pi\left[, H_{t}^{(1)}\left(z^{-1}\right)\right.$ is asymptotic to

$$
\widetilde{H}_{t}^{(1)}\left(z^{-1}\right)=\left(\frac{2 z}{\pi}\right)^{1 / 2} e^{i\left(z^{-1}-t \pi / 2-\pi / 4\right)} \sum_{k=0}^{\infty} \frac{(-1)^{k} \Gamma(t+k+1 / 2) z^{k}}{(2 i)^{k} k ! \Gamma(t-k+1 / 2)} .
$$

Similarly, on the sector $]-2 \pi, \pi\left[, H_{t}^{(2)}\left(z^{-1}\right)\right.$ is asymptotic to

$$
\widetilde{H}_{t}^{(2)}\left(z^{-1}\right)=\left(\frac{2 z}{\pi}\right)^{1 / 2} e^{-i\left(z^{-1}-t \pi / 2-\pi / 4\right)} \sum_{k=0}^{\infty} \frac{\Gamma(t+k+1 / 2) z^{k}}{(2 i)^{k} k ! \Gamma(t-k+1 / 2)} .
$$

It follows that in the basis $\left(H_{t}^{(1)}\left(z^{-1}\right), H_{t}^{(2)}\left(z^{-1}\right)\right)$, the matrix of the monodromy is

$$
\left(\begin{array}{cc}
-1 & 0 \\
0 & -1
\end{array}\right)
$$

and the matrices of the elements of the exponential torus are of the form

$$
\left(\begin{array}{cc}
\alpha & 0 \\
0 & \alpha^{-1}
\end{array}\right), \quad \text { where } \alpha \in \mathbb{C}^{*}
$$

The only level is 1 , and due to the expression of $\widetilde{H}_{t}^{1}\left(z^{-1}\right)$ and $\widetilde{H}_{t}^{2}\left(z^{-1}\right)$, the singular directions are the directions $\pi / 2+k \pi$, with $k \in \mathbb{Z}$. By definition, the Stokes matrix in the direction $\pi / 2+k \pi$ is the matrix that sends the asymptotic representation 
defined on the sector $](k-1) \pi,(k+1) \pi$ [ to the asymptotic representation defined on the sector ] $k \pi,(k+2) \pi$ [. In [Ramis and Martinet 1990, §3.4.12] (see also [Bertrand 1992]), we find that in the basis $\left(H_{t}^{1}\left(z^{-1}\right), H_{t}^{2}\left(z^{-1}\right)\right)$ the Stokes matrix in the direction $\pi / 2+2 k \pi$ is

$$
\left(\begin{array}{cc}
1 & 0 \\
2 e^{2 i \pi t} \cos (\pi t) & 1
\end{array}\right)
$$

and the Stokes matrix in the direction $-\pi / 2+2 k \pi$ is

$$
\left(\begin{array}{cc}
1 & -2 e^{-2 i \pi t} \cos (\pi t) \\
0 & 1
\end{array}\right) \text {. }
$$

An application of the local and global density theorems (Theorems 2.24 and 2.20) gives that

$$
\operatorname{Aut}_{\partial_{z}}^{\Delta_{t}}\left(\left(\mathcal{M}_{U}(z)\right)^{\sim} \mid K_{U, \infty} \cap\left(\mathcal{M}_{U}(z)\right)^{\sim}\right) \quad \text { and } \quad \operatorname{Aut}_{\partial_{z}}^{\Delta_{t}}\left(\left(\mathcal{M}_{U}(z)\right)^{\sim} \mid \mathcal{M}_{U}(z)\right)
$$

are linear differential algebraic subgroups of $\mathrm{SL}_{2}\left(\mathcal{M}_{U}\right)$, because all the matrices we have computed are in $\mathrm{SL}_{2}\left(M_{U}\right)$, which is closed in the Kolchin topology.

Let $C$ be a differentially closed field that contains $\mathcal{M}_{U}$, and consider the parametrized differential Galois group $\mathrm{Gal}_{\partial_{z}}^{\Delta_{t}}\left((C(z))^{\sim} \mid C(z)\right)$ defined in Proposition 2.23. First, we are going to compute the Zariski closure $G$ of $\mathrm{Gal}_{\partial_{z}}^{\Delta_{t}}\left((C(z))^{\sim} \mid C(z)\right)$. Let $C^{*}=C \backslash\{0\}$. From the classification of linear algebraic subgroups of $\mathrm{SL}_{2}(C)$ (see [van der Put and Singer 2003, Theorem 4.29]), there are four possibilities:

(1) $G$ is conjugate to a subgroup of $B=\left\{\left(\begin{array}{cc}a & b \\ 0 & a^{-1}\end{array}\right) \mid a \in C^{*}, b \in \mathbb{C}\right\}$.

(2) $G$ is conjugate to a subgroup of $D_{\infty}=\left\{\left(\begin{array}{cc}a & 0 \\ 0 & a^{-1}\end{array}\right) \mid a \in C^{*}\right\} \cup\left\{\left(\begin{array}{cc}0 & b^{-1} \\ -b & 0\end{array}\right) \mid b \in C^{*}\right\}$.

(3) $G$ is finite.

(4) $G=\mathrm{SL}_{2}(C)$.

From Proposition 2.23, every matrix that belongs to $\operatorname{Aut}_{\partial_{z}}^{\Delta_{t}}\left(\left(\mathcal{M}_{U}(z)\right)^{\sim} \mid \mathcal{M}_{U}(z)\right)$ belongs also to $\mathrm{Gal}_{\partial_{z}}^{\Delta_{t}}\left((C(z))^{\sim} \mid C(z)\right)$. Since $G$ must contain

$$
\left(\begin{array}{cc}
1 & 0 \\
2 e^{2 i \pi t} \cos (\pi t) & 1
\end{array}\right) \text { and }\left(\begin{array}{cc}
1 & -2 e^{-2 i \pi t} \cos (\pi t) \\
0 & 1
\end{array}\right) \text {, }
$$

we find that the only possibility is that $\mathrm{Gal}_{\partial_{z}}^{\Delta_{t}}\left((C(z))^{\sim} \mid C(z)\right)$ is Zariski-dense in $\mathrm{SL}_{2}(C)$. Cassidy [1972, Proposition 42] classified the Zariski-dense differential algebraic subgroups of $\mathrm{SL}_{2}(C)$. Finally, we have two possibilities:

- $\mathrm{Gal}_{\partial_{z}}^{\Delta_{t}}\left((C(z))^{\sim} \mid C(z)\right)$ is conjugate to $\mathrm{SL}_{2}\left(C_{0}\right)$ over $\mathrm{SL}_{2}(C)$, where

$$
C_{0}=\left\{a \in C(z) \mid \partial_{z} a=\partial_{t} a=0\right\} .
$$

- $\mathrm{Gal}_{\partial_{z}}^{\Delta_{t}}\left((C(z))^{\sim} \mid C(z)\right)=\mathrm{SL}_{2}(C)$. 
If $\mathrm{Gal}_{\partial_{z}}^{\Delta_{t}}\left((C(z))^{\sim} \mid C(z)\right)$ is conjugate to $\mathrm{SL}_{2}\left(C_{0}\right)$ over $\mathrm{SL}_{2}(C)$, the matrix of the monodromy of the singularity 0 is conjugate to a matrix $M \in \mathrm{SL}_{2}\left(C_{0}\right)$ over $\mathrm{SL}_{2}(C)$. Similar matrices have the same eigenvalues, so the eigenvalues of $M$ are $e^{2 i \pi t}$ and $e^{-2 i \pi t}$, which is not possible if $M$ belongs to $\mathrm{SL}_{2}\left(C_{0}\right)$. Because of Proposition 2.23, we find that

$$
\operatorname{Aut}_{\partial_{z}}^{\Delta_{t}}\left(\left(M_{U}(z)\right)^{\sim} \mid M_{U}(z)\right)=\operatorname{SL}_{2}\left(M_{U}\right)
$$

\section{Applications}

We now give three applications of parametrized differential Galois theory. In Section 3A, we deal with linear differential equations that are completely integrable (see Definition 3.1). It was proved in [CS] that an equation is completely integrable if and only if its parametrized differential Galois group is conjugate over a differentially closed field to a group of constant matrices. We use the global density theorem (Theorem 2.24) to prove that the equation is completely integrable if and only if there exists a fundamental solution such that the matrices of the topological generators for the Galois group appearing in the global density theorem are constant matrices. As a corollary, we deduce that the equation is completely integrable if and only if the matrices of the topological generators for the Galois group given in the parametrized density theorem are conjugate over $\mathrm{GL}_{m}\left(\mu_{U}\right)$ to constant matrices. In Section 3B, we study an entry of a Stokes operator at the singularity at infinity of the equation

$$
\partial_{z}^{2} Y(z, t)=\left(z^{3}+t\right) Y(z, t) .
$$

In particular, we prove that it is not $\partial_{t}$-finite: it satisfies no parametrized linear differential equation. This partially answers a question by Sibuya. In Section 3C, we deal with the inverse problem in parametrized differential Galois theory. Let $k$ be a so-called universal $\left(\Delta_{t}\right)$-field (see Section $3 \mathrm{~B}$ ). We give a necessary condition for a linear differential algebraic subgroup of $\mathrm{GL}_{m}(k)$ to be the global parametrized differential Galois group for some equation having coefficients in $k(z)$. The corresponding sufficient condition was proved in [Mitschi and Singer 2012, Corollary 5.2].

3A. Completely integrable equations. In this subsection, we study completely integrable equations. See also [Gorchinskiy and Ovchinnikov 2013] for an approach from the point of view of differential Tannakian categories.

Definition 3.1. Let $A_{0} \in \mathrm{M}_{m}\left(M_{U}(z)\right)$. We say that the linear differential equation $\partial_{0} Y=A_{0} Y$ is completely integrable if there exist $A_{1}, \ldots, A_{n} \in \mathrm{M}_{m}\left(M_{U}(z)\right)$ such that, for all $0 \leq i, j \leq n$,

$$
\partial_{t_{i}} A_{j}-\partial_{t_{j}} A_{i}=A_{i} A_{j}-A_{j} A_{i},
$$

with $\partial_{t_{0}}=\partial_{z}$. 
Sibuya [1990, Theorem A.5.2.3] has shown that if the parametrized linear differential equation $(*)$ is regular singular, then it is isomonodromic (see page 89 for the definition) if and only if it is completely integrable. This result is not true in the irregular case. The main reason is the fact that there are more topological generators in the parametrized differential Galois group.

Proposition 3.2. Let $A_{0}(z, t) \in \mathrm{M}_{m}\left(\mathcal{M}_{U}(z)\right)$ and let $\left(\mathcal{M}_{U}(z)\right)^{\sim} \mid \mathcal{M}_{U}(z)$ be the parametrized Picard-Vessiot extension for $\partial_{z} Y(z, t)=A_{0}(z, t) Y(z, t)$ defined in Section $2 E$. The linear differential equation $\partial_{z} Y(z, t)=A_{0}(z, t) Y(z, t)$ is completely integrable if and only if there is a fundamental solution $F(z, t)$ in $\left(M_{U}(z)\right)^{\sim}$ such that the images of the topological generators of $\operatorname{Aut}_{\partial_{z}}^{\Delta_{t}}\left(\left(\mathcal{M}_{U}(z)\right)^{\sim} \mid M_{U}(z)\right)$ (see Theorem 2.24) with respect to the representation associated to $F(z, t)$ belong to $\mathrm{GL}_{m}(\mathbb{C})$.

Proof. Let $C$ be a differentially closed field that contains $\mathcal{M}_{U}$ and let us consider $C(z)$ as in Section 2E. Let $(C(z))^{\sim} \mid C(z)$ be the parametrized Picard-Vessiot extension for $\partial_{z} Y(z, t)=A_{0}(z, t) Y(z, t)$, and let $\mathrm{Gal}_{\partial_{z}}^{\Delta_{t}}\left((C(z))^{\sim} \mid C(z)\right)$ be the parametrized differential Galois group defined in Section 2A. We recall that if we take a different fundamental solution in $\left(M_{U}(z)\right)^{\sim}$ to compute the Galois group, we obtain a conjugate linear differential algebraic subgroup of $\mathrm{GL}_{m}\left(M_{U}\right)$.

Using the global density theorem (Theorem 2.24), we find that there exists a fundamental solution such that the matrices of the topological generators for the Galois group appearing in the global density theorem are constant if and only if $\operatorname{Aut}_{\partial_{z}}^{\Delta_{t}}\left(\left(\mathcal{M}_{U}(z)\right)^{\sim} \mid \mathcal{M}_{U}(z)\right)$ is conjugate over $\mathrm{GL}_{m}\left(\mathcal{M}_{U}\right)$ to a subgroup of $\mathrm{GL}_{m}(\mathbb{C})$. Using Proposition 2.23, we find that $\operatorname{Aut}_{\partial_{z}}^{\Delta_{t}}\left(\left(\mathcal{M}_{U}(z)\right)^{\sim} \mid \mathcal{M}_{U}(z)\right)$ is conjugate over $\mathrm{GL}_{m}\left(M_{U}\right)$ to a subgroup of $\mathrm{GL}_{m}(\mathbb{C})$ if and only if $\mathrm{Gal}_{\partial_{z}}^{\Delta_{t}}\left((C(z))^{\sim} \mid C(z)\right)$ is conjugate over $\mathrm{GL}_{m}(C)$ to a subgroup of $\mathrm{GL}_{m}\left(C_{0}\right)$, where

$$
C_{0}=\left\{a \in C(z) \mid \partial_{z} a=\partial_{t_{1}} a=\cdots=\partial_{t_{n}} a=0\right\} .
$$

Proposition 3.9 of [CS] says that this occurs if and only if there exist $A_{1}, \ldots, A_{n} \in$ $\mathrm{M}_{m}(C(z))$ such that, for all $0 \leq i, j \leq n$,

$$
\partial_{t_{i}} A_{j}-\partial_{t_{j}} A_{i}=A_{i} A_{j}-A_{j} A_{i},
$$

with $\partial_{t_{0}}=\partial_{z}$. To finish, we follow the proof of Proposition 1.24 in [Di Vizio and Hardouin 2012]. Let $0<i \leq n$ and let us consider

$$
\partial_{z} A_{i}-\partial_{t_{i}} A_{0}=A_{0} A_{i}-A_{i} A_{0} .
$$

By clearing the denominators, we obtain that every entry of every $z$-coefficient of $A_{i}$ satisfies a finite set of polynomial equations with coefficients in $M_{U}$. Since the polynomial equations have a solution in $C$, they must have a solution in the algebraic closure of $\mu_{U}$. Using Remark 1.1, we find a nonempty polydisc $U^{\prime} \subset U$ such that all the $A_{i}$ belong to $\mathrm{M}_{m}\left(M_{U^{\prime}}(z)\right)$. This concludes the proof. 
In the proof of Proposition 3.2, we have proved:

Corollary 3.3. Let $A(z, t) \in \mathrm{M}_{m}\left(M_{U}(z)\right)$. The equation (*) is completely integrable if and only if the matrices of the topological generators for the Galois group appearing in Theorem 2.24 are conjugate over $\mathrm{GL}_{m}\left(M_{U}\right)$ to constant matrices.

Remark 3.4. This corollary improves Proposition 3.9 in [CS]. The conjugation occurs in a field that is not differentially closed. Furthermore, we do not need the entire parametrized differential Galois group to be conjugate to a group of constant matrices in order to deduce that the equation $(*)$ is completely integrable.

Gorchinskiy and Ovchinnikov [2013] studied completely integrable parametrized linear differential equations using differential Tannakian categories. In particular, they proved that the notion of integrability with respect to all the parameters is equivalent to the notion of integrability with respect to each parameter separately, which generalizes [Dreyfus 2013, Proposition 9]. Furthermore, they improve Proposition 3.9 in [CS] by avoiding the assumption that the field of constants is differentially closed.

3B. On the hypertranscendence of a Stokes matrix. In this subsection, we will study the parametrized linear differential equation

$$
\partial_{z}^{2} Y(z, t)=\left(z^{3}+t\right) Y(z, t) .
$$

Sibuya [1975, Chapter 2] showed that there exists a formal solution $y_{0}(z, t)$ which admits an asymptotic representation $\tilde{y}_{0}(z, t)$ on the sector

$$
\Sigma(-3 \pi / 5,3 \pi / 5)
$$

(see [ibid., Theorem 6.1]). We easily check that, for $k \in \mathbb{Z}$,

$$
y_{k}(z, t)=y_{0}\left(e^{-2 k i \pi / 5} z, e^{-6 k i \pi / 5} t\right)
$$

is a solution of (3-1) which has the asymptotic representation

$$
\tilde{y}_{k}(z, t)=\tilde{y}_{0}\left(e^{-2 k i \pi / 5} z, e^{-6 k i \pi / 5} t\right)
$$

on the sector $S_{k-1} \cup \bar{S}_{k} \cup S_{k+1}$, where

$$
S_{k}=\Sigma((2 k-1) \pi / 5,(2 k+1) \pi / 5)
$$

and $\bar{S}_{k}$ is its closure.

The asymptotic representation $\tilde{y}_{k}(z, t)$ is bounded uniformly on each compact set in the $t$-plane as $|z|$ tends to infinity on the sector $S_{k}$, and tends to infinity uniformly on each compact set in the $t$-plane as $|z|$ tends to infinity on the sectors $S_{k-1}$ and $S_{k+1}$. As we see in [ibid., p. 83], $y_{k+1}(z, t)$ and $y_{k+2}(z, t)$ are linearly 

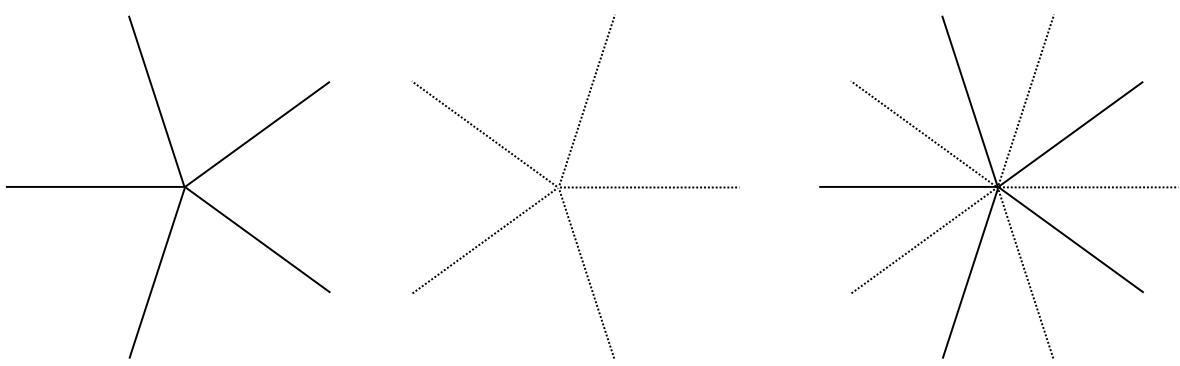

Figure 1. Left: the sectors $S_{k}$. Middle: the singular directions.

Right: the sectors $S_{k}$ and the singular directions.

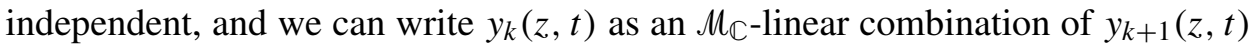
and $y_{k+2}(z, t)$ :

$$
y_{k}(z, t)=C_{k}(t) y_{k+1}(z, t)+\widetilde{C}_{k}(t) y_{k+2}(z, t) \quad \text { for all } k \in \mathbb{N}, z, t \in \mathbb{C},
$$

where $\widetilde{C}_{k}(t), C_{k}(t) \in \mathcal{M}_{\mathbb{C}}$. By Theorem 21.1 in [ibid.], we obtain that

$$
\widetilde{C}_{k}(t)=-e^{2 i \pi / 5} \text { and } C_{k}(t)=C_{0}\left(e^{-6 k i \pi / 5} t\right) .
$$

Sibuya [1975] asked if $C_{0}(t)$ is differentially transcendental, i.e., satisfies no differential polynomial equations. We will use Galois theory to prove that for every nonempty polydisc $U, C_{0}(t)$ is not $\partial_{t}$-finite over $\mu_{U}$, i.e., satisfies no linear differential equations in coefficients in $\mu_{U}$.

The singularity of the system is at infinity. Let $W(z, t)=z Y\left(z^{-1}, t\right)$. We obtain the parametrized linear differential equation

$$
z^{7} \partial_{z}^{2} W(z, t)=\left(1+t z^{3}\right) W(z, t),
$$

which can be written in the form

$$
\partial_{z}\left(\begin{array}{c}
W(z, t) \\
\partial_{z} W(z, t)
\end{array}\right)=\left(\begin{array}{cc}
0 & 1 \\
\frac{1+t z^{3}}{z^{7}} & 0
\end{array}\right)\left(\begin{array}{c}
W(z, t) \\
\partial_{z} W(z, t)
\end{array}\right) .
$$

Let $k$ be a so-called universal $\left(\Delta_{t}\right)$-field of characteristic 0 : for any $\left(\Delta_{t}\right)$-field $k_{0} \subset k,\left(\Delta_{t}\right)$-finitely generated over $\mathbb{Q}$, and any $\left(\Delta_{t}\right)$-finitely generated extension $k_{1}$ of $k_{0}$, there is a $\left(\Delta_{t}\right)$-differential $k_{0}$-isomorphism of $k_{1}$ into $k$. See Chapter $3, \S 7$ of [Kolchin 1973] for more details. In particular, $k$ is $\left(\Delta_{t}\right)$-differentially closed. Let $k(z)$ denote the $\left(\partial_{z}, \Delta_{t}\right)$-differential field of rational functions in the indeterminate $z$ with coefficients in $k$, where $z$ is a $\left(\Delta_{t}\right)$-constant with $\partial_{z} z=1, k$ is the field of constants with respect to $\partial_{z}$, and $\partial_{z}$ commutes with all the derivations.

Let

$$
A(z, t)=\left(\begin{array}{cc}
0 & 1 \\
\frac{1+t z^{3}}{z^{7}} & 0
\end{array}\right) .
$$


The two solutions $z y_{1}\left(z^{-1}, t\right), z y_{2}\left(z^{-1}, t\right)$ admit asymptotic representations and the only singularity is 0 . Therefore,

$$
\mu_{U}(z)\left\langle y_{1}\left(z^{-1}, t\right), y_{2}\left(z^{-1}, t\right)\right\rangle_{\partial_{z}, \partial_{t}}\left|\mathcal{M}_{U}(z)=\left(\mathcal{M}_{U}(z)\right)^{\sim}\right| \mathcal{M}_{U}(z)
$$

is a parametrized Picard-Vessiot extension for $\partial_{z} W(z, t)=A(z, t) W(z, t)$. By Proposition 2.23,

$$
(k(z))^{\sim}\left|k(z)=k(z)\left\langle y_{1}\left(z^{-1}, t\right), y_{2}\left(z^{-1}, t\right)\right\rangle_{\partial_{z}, \partial_{t}}\right| k(z)
$$

is a parametrized Picard-Vessiot extension.

$$
\text { Lemma 3.5. } \quad \operatorname{Gal}_{\partial_{z}}^{\Delta_{t}}\left((k(z))^{\sim} \mid k(z)\right)=\mathrm{SL}_{2}(k) \text {. }
$$

Notice that the differential equation is of the form $\partial_{z}^{2} W(z, t)=r(z, t) W(z, t)$, where $r(z, t) \in k(z)$. In this case, we can compute the Galois group using a parametrized version of Kovacic's algorithm; see [Arreche 2012; Dreyfus 2013]. See also [Acosta-Humanez 2009; Acosta-Humánez et al. 2011]. In order to have a self contained proof, we will perform the calculations explicitly.

Proof. If we apply Kovacic's algorithm [1986], we find that the unparametrized differential Galois group $\mathrm{Gal}_{\partial_{z}}\left((k(z))^{\sim} \mid k(z)\right)$ is equal to $\mathrm{SL}_{2}(k)$. We apply Proposition 6.26 in [Hardouin and Singer 2008], to deduce that $\mathrm{Gal}_{\partial_{z}}^{\Delta_{t}}\left((k(z))^{\sim} \mid k(z)\right)$ is Zariski-dense in $\mathrm{SL}_{2}(k)$. By Proposition 42 in [Cassidy 1972], we deduce that there are two possibilities:

- $\mathrm{Gal}_{\partial_{z}}^{\Delta_{t}}\left((k(z))^{\sim} \mid k(z)\right)=\mathrm{SL}_{2}(k)$

- $\operatorname{Gal}_{\partial_{z}}^{\Delta_{t}}\left((k(z))^{\sim} \mid k(z)\right)$ is conjugate to $\mathrm{SL}_{2}\left(k_{0}\right)$ over $\mathrm{SL}_{2}(k)$, where

$$
k_{0}=\left\{a \in k(z) \mid \partial_{z} a=\partial_{t} a=0\right\} .
$$

We see in [Dreyfus 2013, Remark 4.4] that the last case occurs if and only if the following parametrized differential equation has a solution in $M_{U}(z)$, for some nonempty polydisc $U$ in $\mathbb{C}^{n}$ :

$$
\partial_{z}^{3} y(z, t)=\partial_{z} y(z, t) \frac{4+4 t z^{3}}{z^{7}}+y(z, t) \partial_{z} \frac{4+4 t z^{3}}{z^{7}}-\partial_{t} \frac{4+4 t z^{3}}{z^{7}} .
$$

With the algorithm presented in [van der Put and Singer 2003, p. 100], we find that this does not happen, so

$$
\mathrm{Gal}_{\partial_{z}}^{\Delta_{t}}\left((k(z))^{\sim} \mid k(z)\right)=\mathrm{SL}_{2}(k) .
$$

Lemma 3.6. The singular directions of (3-3) are

$$
\{2 k \pi / 5 \mid k \in \mathbb{Z}\} .
$$


Proof. Let $k \in \mathbb{Z}$. The matrix

$$
\left(\begin{array}{cc}
z y_{k}\left(z^{-1}, t\right) & z y_{k+1}\left(z^{-1}, t\right) \\
\partial_{z} z y_{k}\left(z^{-1}, t\right) & \partial_{z} z y_{k+1}\left(z^{-1}, t\right)
\end{array}\right)
$$

is a fundamental solution for the equation

$$
\partial_{z}\left(\begin{array}{c}
W(z, t) \\
\partial_{z} W(z, t)
\end{array}\right)=\left(\begin{array}{cc}
0 & 1 \\
\frac{1+t z^{3}}{z^{7}} & 0
\end{array}\right)\left(\begin{array}{c}
W(z, t) \\
\partial_{z} W(z, t)
\end{array}\right) .
$$

The fundamental solution admits an asymptotic representation on the sectors

$$
\Sigma((2 k-1) \pi / 5,(2 k+3) \pi / 5) .
$$

The only level is $\frac{5}{2}$. From Proposition 1.13 and the construction of the singular directions, we find that the singular directions are $\{2 k \pi / 5 \mid k \in \mathbb{Z}\}$.

Example 3.7. We want to compute the Stokes matrix in the direction $8 \pi / 5$ for the fundamental solution

$$
\left(\begin{array}{cc}
z y_{1}\left(z^{-1}, t\right) & z y_{2}\left(z^{-1}, t\right) \\
\partial_{z} z y_{1}\left(z^{-1}, t\right) & \partial_{z} z y_{2}\left(z^{-1}, t\right)
\end{array}\right) .
$$

We recall the construction of the Stokes matrices. See Section 1C for the notation. Let $\hat{H}(z, t) z^{L(t)} e(Q(z, t))$ be a fundamental solution in parametrized HukuharaTurrittin canonical form. Let $H^{-}(z, t)$ and $\left.H^{+}(z, t)\right)$ be the matrices such that

$$
H^{-}(z, t) e^{L(t) \log (z)} e^{Q(z, t)} \quad \text { and } \quad H^{+}(z, t) e^{L(t) \log (z)} e^{Q(z, t)}
$$

are the germs of asymptotic solutions on the sectors

$$
\Sigma(\pi, 9 \pi / 5) \text { and } \Sigma(7 \pi / 5,11 \pi / 5),
$$

respectively. The Stokes matrix in the direction $8 \pi / 5$ is the matrix that sends

$$
H^{-}(z, t) e^{L(t) \log (z)} e^{Q(z, t)} \quad \text { to } \quad H^{+}(z, t) e^{L(t) \log (z)} e^{Q(z, t)} .
$$

With the domain of definition of the asymptotic representation of $z \tilde{y}_{1}\left(z^{-1}, t\right)$, we deduce from the definition of the Stokes operators that

$$
\operatorname{St}_{8 \pi / 5}\left(z y_{1}\left(z^{-1}, t\right)\right)=z y_{1}\left(z^{-1}, t\right) .
$$

We first write $\operatorname{St}_{8 \pi / 5}\left(z y_{2}\left(z^{-1}, t\right)\right)$ in the basis

$$
\left(z y_{0}\left(z^{-1}, t\right), z y_{1}\left(z^{-1}, t\right)\right)
$$

There exist $a(t)$ and $b(t) \in \mathcal{M}_{U}$ such that

$$
\operatorname{St}_{8 \pi / 5}\left(z y_{2}\left(z^{-1}, t\right)\right)=a(t) z y_{0}\left(z^{-1}, t\right)+b(t) z y_{1}\left(z^{-1}, t\right) .
$$


By the construction of the asymptotic solutions with Laplace and Borel transforms (see Proposition 1.10), the asymptotic representation of $\operatorname{St}_{8 \pi / 5}\left(z y_{2}\left(z^{-1}, t\right)\right.$ ) has to be bounded in some sector of $] 7 \pi / 5,11 \pi / 5[$, which means that there exist

$$
\frac{7 \pi}{5}<\alpha<\beta<\frac{11 \pi}{5} \text { and } \varepsilon>0
$$

such that $\operatorname{St}_{8 \pi / 5}\left(z y_{2}\left(z^{-1}, t\right)\right)$ is uniformly bounded for $\left.\arg (z) \in\right] \alpha, \beta[$ and $z<|\varepsilon|$. Therefore, $a(t)=0$ or $b(t)=0$. Since the Stokes operators are automorphisms, we get $b(t)=0$. Lemma 3.5 says that the parametrized differential Galois group is $\mathrm{SL}_{2}(k)$. Therefore, because of Proposition 2.23 and Lemma 2.17, the determinant of the matrix has to be 1 . Thus by (3-2), we get that the Stokes matrix in direction $8 \pi / 5$ is

$$
\mathrm{St}_{8 \pi / 5}=\left(\begin{array}{cc}
1 & -C_{0}(t) e^{3 i \pi / 5} \\
0 & 1
\end{array}\right)
$$

Lemma 3.8. Let $C_{0}(t)$ be defined as above. Assume that $C_{0}(t)$ is $\partial_{t}$-finite over $k$. Then the $\partial_{t}$-differential transcendence degree (see Section $2 A$ for definition) of $(k(z))^{\sim}$ over $k(z)$ is at most 2.

Proof. The extension $(k(z))^{\sim}$ is generated over $k(z)$ by $y_{1}\left(z^{-1}, t\right)$ and $y_{2}\left(z^{-1}, t\right)$. By the parametrized differential Galois correspondence (see Theorem 9.5 in $[\mathrm{CS}]$ ), the Kolchin closure of the group generated by $\mathrm{St}_{8 \pi / 5}$ is equal to

$$
\operatorname{Gal}_{\partial_{z}}^{\Delta_{t}}((k(z)) \sim F),
$$

where $F$ is the subfield of $(k(z))^{\sim}$ fixed by $\mathrm{St}_{8 \pi / 5}$. Using (3-4), we deduce that $F$ contains

$$
k(z)\left\langle y_{1}\left(z^{-1}, t\right)\right\rangle_{\partial_{z}, \partial_{t}} .
$$

Because $C_{0}(t)$ satisfies a linear differential equation with coefficients in $k$, there exists a linear differential polynomial $P$ such that this group is of the form

$$
\left\{\left(\begin{array}{ll}
1 & \alpha \\
0 & 1
\end{array}\right) \mid P(\alpha)=0=P\left(C_{0}(t)\right)\right\},
$$

and has $\partial_{t}$-differential dimension over $k$ equal to 0 . Therefore by Proposition 2.7 the $\partial_{t}$-differential transcendence degree of $(k(z))^{\sim}$ over $F$ is equal to 0 . Since $F$ contains $k(z)\left\langle y_{1}\left(z^{-1}, t\right)\right\rangle_{\partial_{z}, \partial_{t}}$, there exists a differential polynomial $Q$ with coefficients in $k(z)$ such that

$$
Q\left(y_{1}\left(z^{-1}, t\right), y_{2}\left(z^{-1}, t\right)\right)=0=Q\left(\partial_{z}\left(y_{1}\left(z^{-1}, t\right)\right), \partial_{z}\left(y_{2}\left(z^{-1}, t\right)\right)\right) .
$$

Therefore, the $\partial_{t}$-differential transcendence degree of $(k(z))^{\sim}$ over $k(z)$ is at most 2 , because $(k(z))^{\sim}$ is generated as a $\partial_{t}$-differential field over $k(z)$ by

$$
\left\{y_{1}\left(z^{-1}, t\right), y_{2}\left(z^{-1}, t\right), \partial_{z}\left(y_{1}\left(z^{-1}, t\right)\right), \partial_{z}\left(y_{2}\left(z^{-1}, t\right)\right)\right\} .
$$


Theorem 3.9. The function $C_{0}(t)$ is not $\partial_{t}$-finite over $k$.

Proof. As we see from Lemma 3.5,

$$
\operatorname{Gal}_{\partial_{z}}^{\Delta_{t}}\left((k(z))^{\sim} \mid k(z)\right)=\mathrm{SL}_{2}(k) .
$$

Therefore, by Proposition 2.7, the $\partial_{t}$-differential transcendence degree of $(k(z))^{\sim}$ over $k(z)$ is 3 . If $C_{0}(t)$ was $\partial_{t}$-finite over $k$, because of Lemma 3.8, the $\partial_{t}$-differential transcendence degree of $(k(z))^{\sim}$ over $k(z)$ would be smaller than 3 . Therefore, $C_{0}(t)$ is not $\partial_{t}$-finite over $k$.

3C. Which linear differential algebraic groups are parametrized differential Galois groups? As in Section 3B, let $k$ be a universal $\left(\Delta_{t}\right)$-field of characteristic 0. Let us consider the equation (*) with $A(z, t) \in \mathrm{M}_{m}(k(z))$, let $(k(z))^{\sim} \mid k(z)$ be the parametrized Picard-Vessiot extension, and let

$$
G=\operatorname{Gal}_{\partial_{z}}^{\Delta_{t}}((k(z)) \sim k(z)) \subset \mathrm{GL}_{m}(k)
$$

be the parametrized differential Galois group defined in Section 2A. The following theorem of Seidenberg, applied with $K_{0}=\mathbb{Q}$ and $K_{1}$ the $\left(\Delta_{t}\right)$-field generated by $\mathbb{Q}$ and the $z$-coefficients of $A(z, t)$, tells us that there exists a nonempty polydisc $U$ such that $A(z, t)$ may be seen as an element of $\mathrm{M}_{m}\left(\mathcal{M}_{U}(z)\right)$.

Theorem 3.10 [Seidenberg 1958; 1969]. Let $\mathbb{Q} \subset K_{0} \subset K_{1}$ be finitely generated $\left(\Delta_{t}\right)$-differential extensions of $\mathbb{Q}$, and assume that $K_{0}$ consists of meromorphic functions on some domain $U$ of $\mathbb{C}^{n}$. Then $K_{1}$ is isomorphic to the field $K_{1}^{*}$ of meromorphic functions on a nonempty polydisc $U^{\prime} \subset U$ such that $\left.K_{0}\right|_{U^{\prime}} \subset K_{1}^{*}$, and the derivations in $\Delta_{t}$ can be identified with the derivations with respect to the coordinates on $U^{\prime}$.

Let $\left(\mathcal{M}_{U}(z)\right)^{\sim} \mid \mathcal{M}_{U}(z)$ be the parametrized Picard-Vessiot extension defined in Section $2 \mathrm{E}$ and let $\operatorname{Aut}_{\partial_{z}}^{\Delta_{t}}\left(\left(\mathcal{M}_{U}(z)\right)^{\sim} \mid \mathcal{M}_{U}(z)\right)$ be the parametrized differential Galois group. Using Corollary 2.25, we find that $\operatorname{Aut}_{\partial_{z}}^{\Delta_{t}}\left(\left(\mathcal{M}_{U}(z)\right)^{\sim} \mid \mathcal{M}_{U}(z)\right)$ contains a finitely generated subgroup that is Kolchin-dense in $\operatorname{Aut}_{\partial_{z}}^{\Delta_{t}}\left(\left(\mathcal{M}_{U}(z)\right)^{\sim} \mid \mathcal{M}_{U}(z)\right)$. With Proposition 2.23, we find that $G$ contains a finitely generated subgroup that is Kolchin-dense in G. Combined with Corollary 5.2 in [Mitschi and Singer 2012], which gives the sufficiency of the condition, this yields the following result:

Theorem 3.11 (inverse problem). Let $G$ be a linear differential algebraic subgroup of $\mathrm{GL}_{m}(k)$. Then $G$ is the global parametrized differential Galois group of some equation having coefficients in $k(z)$ if and only if $G$ contains a finitely generated subgroup that is Kolchin-dense in $G$. 
In the unparametrized case, any linear algebraic group defined over $\mathbb{C}$ is a Galois group of a Picard-Vessiot extension (see [Tretkoff and Tretkoff 1979]). In fact, every linear algebraic group defined over $\mathbb{C}$ contains a finitely generated subgroup that is Zariski-dense, which means that Theorem 3.11 is a generalization of the result in the previous reference.

The situation is more complicated in the parametrized case. For example, the additive group

$$
\left\{\left(\begin{array}{ll}
1 & \alpha \\
0 & 1
\end{array}\right) \mid \alpha \in k\right\}
$$

is not the global parametrized differential Galois group of any equation having coefficients in $k(z)$ (see Section 7 of [CS]). In the parametrized case with only regular singular poles, the problem was solved in [Mitschi and Singer 2012, Corollary 5.2]: they obtain the same necessary and sufficient condition on the group as in Theorem 3.11. Singer [2013] characterized the linear algebraic subgroups of $\mathrm{GL}_{m}(k)$ that appear as the global parametrized differential Galois groups of some equation having coefficients in $k(z)$ : they are the groups such that the identity component has no quotient isomorphic to the additive group $(k,+)$ or multiplicative group $\left(k^{*}, \times\right)$ of $k$.

\section{Appendix}

Let us keep the same notation as in Section 1A and Section 1B. The goal of the appendix is to prove the following theorem. Notice that our proof closely follows the unparametrized case; see [Balser et al. 1980; Loday-Richaud 2001]. See Remark 1.6 for a discussion of another similar result.

Theorem A.1. Consider the equation $\partial_{z} Y(z, t)=A(z, t) Y(z, t)$ with $A(z, t) \in$ $\mathrm{M}_{m}\left(\hat{K}_{U}\right)$. There exists a nonempty polydisc $U^{\prime} \subset U$ such that we have a fundamental solution of the form

$$
\hat{P}(z, t) z^{C(t)} e(Q(z, t)) \in \mathrm{GL}_{m}\left(\left(\mathbf{K}_{U^{\prime}}\right)^{\wedge}\right),
$$

with:

- $\hat{P}(z, t) \in \mathrm{GL}_{m}\left(\hat{K}_{U^{\prime}}\right)$,

- $C(t) \in \mathrm{M}_{m}\left(M_{U^{\prime}}\right)$,

- $e(Q(z, t))=\operatorname{Diag}\left(e\left(q_{i}(z, t)\right)\right)$, with $q_{i}(z, t) \in \mathbf{E}_{U^{\prime}}$.

Moreover, we may choose the same nonempty polydisc $U^{\prime}$ as in Proposition 1.3. Combined with Remark 1.6, if $A(z, t) \in \mathrm{M}_{m}\left(\mathscr{O}_{U}(\{z\})\right)$, this gives a sufficient condition on $t_{0} \in U$ to have a fundamental solution $\hat{P}(z, t) z^{C(t)} e(Q(z, t)) \in \operatorname{GL}_{m}\left(\left(\mathbf{K}_{U^{\prime}}\right)^{\wedge}\right)$ in the same form as above with $t_{0} \in U^{\prime}$. 
Remark that, contrary to Proposition $1.3, \hat{H}(z, t) \in \mathrm{GL}_{m}\left(\hat{K}_{U^{\prime}}\right)$. On the other hand, we lose the commutation between $z^{C(t)}$ and $e(Q(z, t))$. Before giving the proof of the theorem, we state and prove two lemmas.

Lemma A.2. Let $U^{\prime} \subset U$ be a nonempty polydisc. Let $a(t) \in M_{U^{\prime}}$ and $\alpha(z, t) \in$ $\hat{K}_{F, U^{\prime}}$ such that $\hat{m}(\alpha(z, t))=a(t) \alpha(z, t)$. Then there exist $\hat{h}(z, t) \in \hat{K}_{U^{\prime}}$ and $b(t) \in M_{U^{\prime}}$ such that $\alpha(z, t)=\hat{h}(z, t) z^{b(t)}$.

Proof. Let $\alpha(z, t) \in \hat{K}_{F, U^{\prime}}$ such that $\hat{m}(\alpha(z, t))=a(t) \alpha(z, t)$. The element $\alpha(z, t)$ belongs to the fraction field of a free polynomial ring

$$
P=\hat{K}_{U^{\prime}}\left[\log , z^{b_{1}(t)}, \ldots, z^{b_{k}(t)}\right] .
$$

Write $\alpha(z, t)=\alpha_{1}(z, t) / \alpha_{2}(z, t)$, where $\alpha_{1}$ and $\alpha_{2}$ have gcd 1 in $P$. Using the relations in $\hat{K}_{F, U^{\prime}}$, and applying $\hat{m}$ to $\alpha_{1}(z, t) / \alpha_{2}(z, t)$, we find that $\alpha(z, t)$ contains no terms in log. One can normalize $\alpha_{2}(z, t)$ such that it contains a term of the form $z^{n_{1} b_{1}(t)+\cdots+n_{k} b_{k}(t)}$ with coefficient 1 and $n_{i} \in \mathbb{Z}$. Using

$$
\hat{m}\left(\alpha_{1}(z, t) / \alpha_{2}(z, t)\right)=a(t) \alpha_{1}(z, t) / \alpha_{2}(z, t),
$$

we find that

$$
\hat{m}\left(\alpha_{2}(z, t)\right)=e^{2 i \pi\left(n_{1} b_{1}(t)+\cdots+n_{k} b_{k}(t)\right)} \alpha_{2}(z, t)
$$

and

$$
\hat{m}\left(\alpha_{1}(z, t)\right)=a(t) e^{2 i \pi\left(n_{1} b_{1}(t)+\cdots+n_{1} b_{1}(t)\right)} \alpha_{1}(z, t),
$$

which is impossible unless

$$
e^{2 i \pi\left(n_{1} b_{1}(t)+\cdots+n_{k} b_{k}(t)\right)}=1 .
$$

This means that $\alpha_{2}(z, t) \in \hat{K}_{U^{\prime}}$ and we may assume $\alpha_{2}(z, t)=1$. Applying $\hat{m}$ to $\alpha_{1}(z, t)$, one finds that $\alpha_{1}(z, t)$ contains at most one term, that is, $\alpha(z, t)=$ $\hat{h}(z, t) z^{b(t)}$, with $\hat{h}(z, t) \in \hat{K}_{U^{\prime}}$ and $b(t) \in M_{U^{\prime}}$ satisfying $e^{2 i \pi b(t)}=a(t)$.

Lemma A.3. Let $U^{\prime} \subset U$ be a nonempty polydisc. Let $A(z, t) \in \mathrm{M}_{m}\left(\hat{K}_{U^{\prime}}\right)$. Let $F_{1}(z, t) e\left(Q_{1}(z, t)\right)$ and $F_{2}(z, t) e\left(Q_{2}(z, t)\right)$ be two fundamental solutions of the equation $(*)$ such that, for $i=1,2$, we have

$$
F_{i}(z, t) \in \mathrm{GL}_{m}\left(\hat{K}_{F, U^{\prime}}\right) \text { and } Q_{i}(z, t)=\operatorname{Diag}\left[q_{i, j}(z, t)\right],
$$

where the $q_{i, j}(z, t)$ belong to $\mathbf{E}_{U^{\prime}}$. Then $F_{1}(z, t)^{-1} F_{2}(z, t) \in \mathrm{GL}_{m}\left(\mathcal{M}_{U^{\prime}}\right)$.

Proof. A straightforward computation shows that

$$
\partial_{z} g\left(\left(F_{1}(z, t) e\left(Q_{1}(z, t)\right)\right)^{-1} F_{2}(z, t) e\left(Q_{2}(z, t)\right) g\right)=0 .
$$

By Proposition 2.19,

$$
\left(F_{1}(z, t) e\left(Q_{1}(z, t)\right)\right)^{-1} F_{2}(z, t) e\left(Q_{2}(z, t)\right)=C(t) \in \mathrm{GL}_{m}\left(M_{U^{\prime}}\right) .
$$


Hence, we have the equality

$$
e\left(Q_{1}(z, t)\right) C(t) e\left(-Q_{2}(z, t)\right)=F_{1}(z, t)^{-1} F_{2}(z, t) .
$$

The entries of $e\left(Q_{1}(z, t)\right) C(t) e\left(-Q_{2}(z, t)\right)$ are of the form

$$
C_{i, j}(t) e\left(q_{1, j}(z, t)-q_{2, j}(z, t)\right),
$$

with $C_{i, j}(t) \in M_{U^{\prime}}$, and the matrix $F_{1}(z, t)^{-1} F_{2}(z, t)$ belongs to $\operatorname{GL}_{m}\left(\hat{K}_{F, U^{\prime}}\right)$. By construction, $\hat{K}_{F, U^{\prime}} \cap \mathcal{M}_{U^{\prime}}\left((e(q(z, t)))_{q(z, t) \in \mathbf{E}_{U^{\prime}}}\right)=\mathcal{M}_{U^{\prime}}$, and we obtain

$$
F_{1}(z, t)^{-1} F_{2}(z, t) \in \mathrm{GL}_{m}\left(M_{U^{\prime}}\right) \text {. }
$$

Proof of Theorem A.1. By Proposition 1.3, we know that we have a fundamental solution of the parametrized linear differential equation $(*)$ of the form

$$
\hat{H}(z, t) z^{L(t)} e(Q(z, t)),
$$

with $\hat{H}(z, t) \in \mathrm{GL}_{m}\left(\hat{K}_{U^{\prime}}\left[z^{1 / \nu}\right]\right)$ and $v \in \mathbb{N}^{*}$. From Definition 2.13, $\hat{m}$ commutes with the derivation $\partial_{z}$, and therefore $\hat{m}\left(\hat{H}(z, t) z^{L(t)} e(Q(z, t))\right)$ is another fundamental solution. From the construction of $\hat{m}$, we deduce that $\hat{m}\left(\hat{H}(z, t) z^{L(t)}\right) \in \mathrm{GL}_{m}\left(\hat{K}_{F, U^{\prime}}\right)$, and we can apply Lemma A.3 to deduce the existence of $\hat{M}(t) \in \mathrm{GL}_{m}\left(\mathcal{M}_{U^{\prime}}\right)$ such that

$$
\hat{m}\left(\hat{H}(z, t) z^{L(t)}\right)=\hat{H}(z, t) z^{L(t)} \hat{M}(t) .
$$

Let us consider $\hat{M}(t)=D(t) U(t)$, with $D(t)$ diagonalizable and $U(t)$ unipotent such that $D(t) U(t)=U(t) D(t)$ is the multiplicative analogue of the Jordan decomposition of $\hat{M}(t)$. If $a(t)$ is an eigenvalue of $D(t)$ (and therefore an eigenvalue of $\hat{M}(t))$, then there exists $0 \neq \alpha(z, t) \in \hat{K}_{F, U^{\prime}}$ such that $\hat{m}(\alpha(z, t))=a(t) \alpha(z, t)$, because of the relation (A-1). By Lemma A.2, $\alpha(z, t)$ is equal to $\hat{h}(z, t) z^{b(t)}$, with $b(t) \in \mathcal{M}_{U^{\prime}}$ satisfying $e^{2 i \pi b(t)}=a(t)$ and $\hat{h}(z, t) \in \hat{K}_{U^{\prime}}$. This implies that $a(t)$ and all the eigenvalues of $D(t)$ are of the form $e^{\beta(t)}$, with $\beta(t) \in \mathcal{M}_{U^{\prime}}$. So we have proved the existence of $C(t) \in \mathrm{M}_{m}\left(M_{U^{\prime}}\right)$ such that $e^{2 i \pi C(t)}=\hat{M}(t)$. Let

$$
\hat{P}(z, t)=\hat{H}(z, t) z^{L(t)} z^{-C(t)} .
$$

A computation shows that the monodromy of $z^{C(t)}$ is

$$
\hat{m}\left(z^{C(t)}\right)=e^{2 i \pi C(t)} z^{C(t)}=z^{C(t)} e^{2 i \pi C(t)} .
$$

The matrix $\hat{P}(z, t)$ is fixed by the monodromy and therefore belongs to $\mathrm{GL}_{m}\left(\hat{K}_{U^{\prime}}\right)$, by Proposition 2.19. Finally,

$$
\hat{P}(z, t) z^{C(t)} e(Q(z, t))
$$

is a fundamental solution of the parametrized linear differential equation $(*)$ that has the required property. 


\section{Acknowledgements}

This paper was prepared during my thesis, supported by the region Île de France. I want to thank my advisor Lucia Di Vizio for her helpful comments and the interesting discussions we had during the preparation of this paper. I also want to thank the organizers of the seminars that have made it possible for me to present the results contained in this paper. I want to thank Jean-Pierre Ramis, Guy Casale, Reinhard Schäfke, Daniel Bertrand and Michael F. Singer for pointing some mistakes and inaccuracies in this paper. Michael F. Singer in particular suggested the contribution of Theorem 3.11 to this paper. I certainly thank Carlos E. Arreche and Claude Mitschi for the read-through. Lastly, I heartily thank the anonymous referees of the two successive submissions and Jacques Sauloy, who spent a great lot of time and effort to help me make this paper readable.

\section{References}

[Acosta-Humanez 2009] P. B. Acosta-Humanez, Galoisian Approach to Supersymmetric Quantum Mechanics, Phd Dissertation, Ph.D. thesis, Universitat Politecnica de Catalunya, 2009.

[Acosta-Humánez et al. 2011] P. B. Acosta-Humánez, J. J. Morales-Ruiz, and J.-A. Weil, "Galoisian approach to integrability of Schrödinger equation”, Rep. Math. Phys. 67:3 (2011), 305-374. MR 2846216 Zbl 1238.81090

[Arreche 2012] C. E. Arreche, "Computing the differential galois group of a one-parameter family of second order linear differential equations”, preprint, 2012. arXiv 1208.2226

[Babbitt and Varadarajan 1985] D. G. Babbitt and V. S. Varadarajan, "Deformations of nilpotent matrices over rings and reduction of analytic families of meromorphic differential equations", 325 (1985), iv+147. MR 87i:12014 Zbl 0583.34007

[Balser 1994] W. Balser, From divergent power series to analytic functions, Lecture Notes in Mathematics 1582, Springer, Berlin, 1994. MR 96d:34071 Zbl 0810.34046

[Balser et al. 1980] W. Balser, W. B. Jurkat, and D. A. Lutz, "A general theory of invariants for meromorphic differential equations, III: Applications”, Houston J. Math. 6:2 (1980), 149-189. MR 83m:34003c Zbl 0506.34006

[Bertrand 1992] D. Bertrand, "Groupes algébriques et équations différentielles linéaires”, 206 (1992), 183-204. MR 94b:34006 Zbl 0813.12004

[Bolibruch 1997] A. A. Bolibruch, "On isomonodromic deformations of Fuchsian systems", J. Dynam. Control Systems 3:4 (1997), 589-604. MR 99c:34003 Zbl 0943.34083

[Cano and Ramis 1995] J. Cano and J.-P. Ramis, "Théorie de Galois différentielle, multisommabilité et phénomène de Stokes", notes from the course Journées Galois Différentielles conducted in May 1993 at Toulouse by J.-P. Ramis and M. Loday-Richaud, 1995, http://www.math.univ-toulouse.fr/ $\sim$ ramis/Cano-Ramis-Galois.pdf.

[Cassidy 1972] P. J. Cassidy, "Differential algebraic groups", Amer. J. Math. 94 (1972), 891-954. MR 50 \#13058 Zbl 0258.14013

[Cassidy 1989] P. J. Cassidy, "The classification of the semisimple differential algebraic groups and the linear semisimple differential algebraic Lie algebras", J. Algebra 121:1 (1989), 169-238. MR 90g:12007 Zbl 0678.14011 
[Cassidy and Singer 2007] P. J. Cassidy and M. F. Singer, "Galois theory of parameterized differential equations and linear differential algebraic groups", pp. 113-155 in Differential equations and quantum groups, IRMA Lect. Math. Theor. Phys. 9, Eur. Math. Soc., Zürich, 2007. MR 2008f:12010 Zbl 1230.12003

[Chatzidakis et al. 2008] Z. Chatzidakis, C. Hardouin, and M. F. Singer, "On the definitions of difference Galois groups", pp. 73-109 in Model theory with applications to algebra and analysis, I, edited by Z. Chatzidakis et al., London Math. Soc. Lecture Note Ser. 349, Cambridge Univ. Press, 2008. MR 2009j:12014 Zbl 1234.12005

[Di Vizio and Hardouin 2012] L. Di Vizio and C. Hardouin, "Descent for differential Galois theory of difference equations: confluence and q-dependence", Pacific J. Math. 256:1 (2012), 79-104. MR 2928542 Zbl 1258.12004

[Dreyfus 2013] T. Dreyfus, "Computing the Galois group of some parameterized linear differential equation of order two", preprint, 2013. To appear in Proc. Amer. Math. Soc. arXiv 1110.1053

[Écalle 1981] J. Écalle, Les fonctions résurgentes, I, Publications Mathématiques d'Orsay 81-05, Université de Paris-Sud Département de Mathématique, Orsay, 1981. MR 84h:30077a Zbl 0499.30034

[Gillet et al. 2013] H. Gillet, S. Gorchinskiy, and A. Ovchinnikov, "Parameterized Picard-Vessiot extensions and Atiyah extensions", Adv. Math. 238 (2013), 322-411. MR 3033637

[Gorchinskiy and Ovchinnikov 2013] S. Gorchinskiy and A. Ovchinnikov, "Isomonodromic differential equations and differential tannakian categories", preprint, 2013.

[Hardouin and Singer 2008] C. Hardouin and M. F. Singer, "Differential Galois theory of linear difference equations”, Math. Ann. 342:2 (2008), 333-377. MR 2009j:39001 Zbl 1163.12002

[Kolchin 1973] E. R. Kolchin, Differential algebra and algebraic groups, Pure and Applied Mathematics 54, Academic Press, New York, 1973. MR 58 \#27929 Zbl 0264.12102

[Kolchin 1985] E. R. Kolchin, Differential algebraic groups, Pure and Applied Mathematics 114, Academic Press, Orlando, FL, 1985. MR 87i:12016 Zbl 0556.12006

[Kovacic 1986] J. J. Kovacic, "An algorithm for solving second order linear homogeneous differential equations", J. Symbolic Comput. 2:1 (1986), 3-43. MR 88c:12011 Zbl 0603.68035

[Landesman 2008] P. Landesman, "Generalized differential Galois theory", Trans. Amer. Math. Soc. 360:8 (2008), 4441-4495. MR 2009i:12005 Zbl 1151.12004

[Loday-Richaud 1990] M. Loday-Richaud, "Introduction à la multisommabilité", Gaz. Math. 44 (1990), 41-63. MR 91h:40007 Zbl 0722.34005

[Loday-Richaud 1994] M. Loday-Richaud, "Stokes phenomenon, multisummability and differential Galois groups", Ann. Inst. Fourier (Grenoble) 44:3 (1994), 849-906. MR 95g:34010 Zbl 0812.34004

[Loday-Richaud 1995] M. Loday-Richaud, "Solutions formelles des systèmes différentiels linéaires méromorphes et sommation", Exposition. Math. 13:2-3 (1995), 116-162. MR 96i:34124 Zbl 0831.34002

[Loday-Richaud 2001] M. Loday-Richaud, "Rank reduction, normal forms and Stokes matrices", Expo. Math. 19:3 (2001), 229-250. MR 2002k:34173 Zbl 0990.34076

[Loday-Richaud and Remy 2011] M. Loday-Richaud and P. Remy, "Resurgence, Stokes phenomenon and alien derivatives for level-one linear differential systems", J. Differential Equations 250:3 (2011), 1591-1630. MR 2011m:34264 Zbl 1214.34087

[Magid 1994] A. R. Magid, Lectures on differential Galois theory, University Lecture Series 7, Amer. Math. Soc., Providence, RI, 1994. MR 95j:12008 Zbl 0855.12001 
[Malgrange 1983] B. Malgrange, "Sur les déformations isomonodromiques, II: Singularités irrégulières", pp. 427-438 in Mathematics and physics (Paris, 1979/1982), edited by L. Boutet de Monvel et al., Progr. Math. 37, Birkhäuser, Boston, MA, 1983. MR 85m:58094b Zbl 0528.32018

[Malgrange 1991] B. Malgrange, Équations différentielles à coefficients polynomiaux, Progress in Mathematics 96, Birkhäuser, Boston, MA, 1991. MR 92k:32020 Zbl 0764.32001

[Malgrange 1995] B. Malgrange, "Sommation des séries divergentes", Exposition. Math. 13:2-3 (1995), 163-222. MR 96i:34125 Zbl 0836.40004

[Malgrange and Ramis 1992] B. Malgrange and J.-P. Ramis, "Fonctions multisommables", Ann. Inst. Fourier (Grenoble) 42:1-2 (1992), 353-368. MR 93e:40007 Zbl 0759.34007

[Minchenko and Ovchinnikov 2011] A. Minchenko and A. Ovchinnikov, "Zariski closures of reductive linear differential algebraic groups", Adv. Math. 227:3 (2011), 1195-1224. MR 2012k:12010 Zbl 1215.12009

[Mitschi 1996] C. Mitschi, "Differential Galois groups of confluent generalized hypergeometric equations: An approach using Stokes multipliers", Pacific J. Math. 176:2 (1996), 365-405. MR 98f:12005 Zbl 0883.12004

[Mitschi and Singer 2012] C. Mitschi and M. F. Singer, "Monodromy groups of parameterized linear differential equations with regular singularities", Bull. Lond. Math. Soc. 44:5 (2012), 913-930. MR 2975151 Zbl 1254.34124

[Mitschi and Singer 2013] C. Mitschi and M. F. Singer, "Projective isomonodromy and Galois groups”, Proc. Amer. Math. Soc. 141:2 (2013), 605-617. MR 2996965 Zbl 1268.34187

[Peón Nieto 2011] A. Peón Nieto, "On $\sigma \delta$-Picard-Vessiot extensions", Comm. Algebra 39:4 (2011), 1242-1249. MR 2012c:12011 Zbl 1272.12018

[van der Put and Singer 2003] M. van der Put and M. F. Singer, Galois theory of linear differential equations, Grundlehren der Mathematischen Wissenschaften 328, Springer, Berlin, 2003. MR 2004c:12010 Zbl 1036.12008

[Ramis 1980] J.-P. Ramis, "Les séries k-sommables et leurs applications", pp. 178-199 in Complex analysis, microlocal calculus and relativistic quantum theory (Les Houches, 1979), edited by D. Iagolnitzer, Lecture Notes in Phys. 126, Springer, Berlin, 1980. MR 82k:32033 Zbl 1251.32008

[Ramis 1985] J.-P. Ramis, "Phénomène de Stokes et filtration Gevrey sur le groupe de Picard-Vessiot", C. R. Acad. Sci. Paris Sér. I Math. 301:5 (1985), 165-167. MR 86k:12012 Zbl 0593.12015

[Ramis and Martinet 1990] J.-P. Ramis and J. Martinet, "Théorie de Galois différentielle et resommation", pp. 117-214 in Computer algebra and differential equations, edited by E. Tournier, Academic Press, London, 1990. MR 91d:12014 Zbl 0722.12007

[Ramis and Sibuya 1989] J.-P. Ramis and Y. Sibuya, "Hukuhara domains and fundamental existence and uniqueness theorems for asymptotic solutions of Gevrey type", Asymptotic Anal. 2:1 (1989), 39-94. MR 90k:58209 Zbl 0699.34058

[Rasoamanana 2010] J.-M. Rasoamanana, "Résurgence-sommabilité de séries formelles ramifiées dépendant d'un paramètre et solutions d'équations différentielles linéaires", Ann. Fac. Sci. Toulouse Math. (6) 19:2 (2010), 303-343. MR 2011g:34207 Zbl 1205.34122

[Remy 2012] P. Remy, "Matrices de Stokes-Ramis et constantes de connexion pour les systèmes différentiels linéaires de niveau unique", Ann. Fac. Sci. Toulouse Math. (6) 21:1 (2012), 93-150. MR 2954106 Zbl 1244.34109

[Robinson 1959] A. Robinson, "On the concept of a differentially closed field", Bull. Res. Council Israel Sect. F 8F (1959), 113-128. MR 23 \#A2323 Zbl 0221.12054 
[Schäfke 2001] R. Schäfke, "Formal fundamental solutions of irregular singular differential equations depending upon parameters", J. Dynam. Control Systems 7:4 (2001), 501-533. MR 2002g:34199 Zbl 1029.34077

[Seidenberg 1958] A. Seidenberg, "Abstract differential algebra and the analytic case", Proc. Amer. Math. Soc. 9 (1958), 159-164. MR 20 \#178 Zbl 0186.07502

[Seidenberg 1969] A. Seidenberg, "Abstract differential algebra and the analytic case, II", Proc. Amer. Math. Soc. 23 (1969), 689-691. MR 40 \#1376 Zbl 0186.07503

[Sibuya 1975] Y. Sibuya, Global theory of a second order linear ordinary differential equation with a polynomial coefficient, North-Holland Mathematics Studies 18, North-Holland, Amsterdam, 1975. MR 58 \#6561 Zbl 0322.34006

[Sibuya 1990] Y. Sibuya, Linear differential equations in the complex domain: Problems of analytic continuation, Translations of Mathematical Monographs 82, Amer. Math. Soc., Providence, RI, 1990. MR 92a:34010 Zbl 1145.34378

[Singer 2009] M. F. Singer, "Introduction to the Galois theory of linear differential equations", pp. 1-82 in Algebraic theory of differential equations (Edinburgh, 2006), edited by M. A. H. MacCallum and A. V. Mikhailov, London Math. Soc. Lecture Note Ser. 357, Cambridge Univ. Press, 2009. MR 2010d:12006 Zbl 1176.12005

[Singer 2013] M. F. Singer, "Linear algebraic groups as parameterized Picard-Vessiot Galois groups", J. Algebra 373 (2013), 153-161. MR 2995020 Zbl 06182880

[Tretkoff and Tretkoff 1979] C. Tretkoff and M. Tretkoff, "Solution of the inverse problem of differential Galois theory in the classical case", Amer. J. Math. 101:6 (1979), 1327-1332. MR 80k:12033 Zbl 0423.12021

[Umemura 1996] H. Umemura, "Galois theory of algebraic and differential equations", Nagoya Math. J. 144 (1996), 1-58. MR 98c:12009 Zbl 0885.12004

[Wasow 1965] W. Wasow, Asymptotic expansions for ordinary differential equations, Pure and Applied Mathematics 14, Interscience, New York, 1965. Reprinted Dover, New York, 1987. MR 34 \#3041 Zbl 0133.35301

[Watson 1944] G. N. Watson, A treatise on the theory of Bessel functions, 2nd ed., Cambridge University Press, Cambridge, England, 1944. Reprinted Cambridge, 1995. MR 6,64a Zbl 0174.36202

[Wibmer 2012] M. Wibmer, "Existence of $\partial$-parameterized Picard-Vessiot extensions over fields with algebraically closed constants”, J. Algebra 361 (2012), 163-171. MR 2921616 Zbl 06125724

Received May 21, 2013.

THOMAS DREYFUS

InSTITUT DE MAThÉMATiQUes DE JUSSiEU

UNIVERSITÉ PARIS DIDEROT

4, PLACE JUSSIEU

75005 PARIS

FRANCE

thomas.dreyfus@imj-prg.fr 


\title{
PACIFIC JOURNAL OF MATHEMATICS
}

\author{
msp.org/pjm
}

Founded in 1951 by E. F. Beckenbach (1906-1982) and F. Wolf (1904-1989)

\section{EDITORS}

Don Blasius (Managing Editor)

Department of Mathematics

University of California

Los Angeles, CA 90095-1555

blasius@math.ucla.edu

\author{
Paul Balmer \\ Department of Mathematics \\ University of California \\ Los Angeles, CA 90095-1555 \\ balmer@math.ucla.edu \\ Robert Finn \\ Department of Mathematics \\ Stanford University \\ Stanford, CA 94305-2125 \\ finn@math.stanford.edu \\ Sorin Popa \\ Department of Mathematics \\ University of California \\ Los Angeles, CA 90095-1555 \\ popa@math.ucla.edu
}

\author{
Vyjayanthi Chari \\ Department of Mathematics \\ University of California \\ Riverside, CA 92521-0135 \\ chari@math.ucr.edu \\ Kefeng Liu \\ Department of Mathematics \\ University of California \\ Los Angeles, CA 90095-1555 \\ liu@math.ucla.edu \\ Jie Qing \\ Department of Mathematics \\ University of California \\ Santa Cruz, CA 95064 \\ qing@ cats.ucsc.edu
}

\section{PRODUCTION}

Silvio Levy, Scientific Editor, production@msp.org

\section{SUPPORTING INSTITUTIONS}

ACADEMIA SINICA, TAIPEI

CALIFORNIA INST. OF TECHNOLOGY

INST. DE MATEMÁTICA PURA E APLICADA

KEIO UNIVERSITY

MATH. SCIENCES RESEARCH INSTITUTE

NEW MEXICO STATE UNIV.

OREGON STATE UNIV.

\author{
STANFORD UNIVERSITY \\ UNIV. OF BRITISH COLUMBIA \\ UNIV. OF CALIFORNIA, BERKELEY \\ UNIV. OF CALIFORNIA, DAVIS \\ UNIV. OF CALIFORNIA, LOS ANGELES \\ UNIV. OF CALIFORNIA, RIVERSIDE \\ UNIV. OF CALIFORNIA, SAN DIEGO \\ UNIV. OF CALIF., SANTA BARBARA
}

\author{
Daryl Cooper \\ Department of Mathematics \\ University of California \\ Santa Barbara, CA 93106-3080 \\ cooper@math.ucsb.edu \\ Jiang-Hua Lu \\ Department of Mathematics \\ The University of Hong Kong \\ Pokfulam Rd., Hong Kong \\ jhlu@maths.hku.hk \\ Paul Yang \\ Department of Mathematics \\ Princeton University \\ Princeton NJ 08544-1000 \\ yang@math.princeton.edu
}

These supporting institutions contribute to the cost of publication of this Journal, but they are not owners or publishers and have no responsibility for its contents or policies.

See inside back cover or msp.org/pjm for submission instructions.

The subscription price for 2014 is US $\$ 410 /$ year for the electronic version, and \$535/year for print and electronic.

Subscriptions, requests for back issues and changes of subscribers address should be sent to Pacific Journal of Mathematics, P.O. Box 4163, Berkeley, CA 94704-0163, U.S.A. The Pacific Journal of Mathematics is indexed by Mathematical Reviews, Zentralblatt MATH, PASCAL CNRS Index, Referativnyi Zhurnal, Current Mathematical Publications and Web of Knowledge (Science Citation Index).

The Pacific Journal of Mathematics (ISSN 0030-8730) at the University of California, c/o Department of Mathematics, 798 Evans Hall \#3840, Berkeley, CA 94720-3840, is published twelve times a year. Periodical rate postage paid at Berkeley, CA 94704, and additional mailing offices. POSTMASTER: send address changes to Pacific Journal of Mathematics, P.O. Box 4163, Berkeley, CA 94704-0163.

PJM peer review and production are managed by EditFLOW ${ }^{\circledR}$ from Mathematical Sciences Publishers.

\section{PUBLISHED BY}

\section{mathematical sciences publishers \\ nonprofit scientific publishing}

http://msp.org/

(C) 2014 Mathematical Sciences Publishers 


\section{PACIFIC JOURNAL OF MATHEMATICS}

Volume $271 \quad$ No. $1 \quad$ September 2014

Proper holomorphic maps between bounded symmetric domains revisited 1

GAUTAM BHARALI and JAIKRISHNAN JANARDHANAN

An explicit Majorana representation of the group $3^{2}: 2$ of $3 C$-pure type 25

HSIAN-YANG CHEN and CHING HUNG LAM

Sofic groups: graph products and graphs of groups

LAUra Ciobanu, DereK F. Holt and SARAh ReES

Perturbations of a critical fractional equation

Eduardo Colorado, Arturo de Pablo and Urko SÁnchez

A density theorem in parametrized differential Galois theory

THOMAS DREYFUS

On the classification of complete area-stationary and stable surfaces in $\quad 143$ the subriemannian Sol manifold

MATteo Galli

Periodic orbits of Hamiltonian systems linear and hyperbolic at infinity

BAŞAK Z. GÜREL

Nonsplittability of the rational homology cobordism group of

3-manifolds

SE-Goo KIM and CHARLES LIVINGSTON

Biharmonic surfaces of constant mean curvature

ERIC LOUBEAU and CEZAR ONICIUC

Foliations of a smooth metric measure space by hypersurfaces with constant $f$-mean curvature

JUNCHEOL PYO

On the existence of large degree Galois representations for fields of small 243 discriminant

JEREMY ROUSE and FRANK THORNE 